\title{
Interactive Development of Seals, Bearings, and Secondary Flow Systems with the Power Stream
}

\author{
R. C. HENDRICKS and B. M. STEINETZ \\ National Aeronautics and Space Administration, Lewis Research Center, Cleveland, Ohio 44135 \\ M. M. ATHAVALE and A. J. PRZEKWAS \\ CFDRC, Huntsville, Alabama 35805 \\ M. J. BRAUN, M. I. DOZOZO, F. K. CHOY and V. V. KUDRIAVTSEV \\ University of Akron, Akron, Ohio 44315 \\ R. L. MULLEN \\ Case Western Reserve University, Cleveland, Ohio 44106 \\ G. L. VON PRAGENAU \\ 3509 Mae Drive, Huntsville, Alabama 35801
}

\section{INTRODUCTION}

$\mathbf{T}$ Thermodynamic engine cycles, such as turbojets, turbofans, and rocket engine gas-generators, expanders, and staged combustors, are modeled to establish engine component parameters (Oates [1984], Huzal and Huang [1992], and Sutton [1986]). Each original equipment manufacturer (e.g., Rocketdyne, Aerojet, Pratt \& Whitney, General Electric, and Allison) has such an engine cycle model as well as a more detailed engine model involving the propellant, oxidizer, and combustion flow streams that essentially define the engine operating envelope and the overall engine power, fuel and oxidizer consumption, losses, and staging parameters. Details of these models are held proprietary with current efforts directed toward numerical simulation (Claus et al. [1991] and Nichols and Chamis [1991]).

Component modeling of the turbomachinery (pump, compressor, or turbine), combustor, and control cycle sequencing defines the subcomponent hardware, although most designs are perturbations on hardware with significant operational history. Bearings and seals represent subcomponents of turbomachines that are necessary for function through support, fluid control, and dynamic behavior. Rolling-element bearings are most frequently used and require lubricants that in turn require either an auxiliary fluid loop or special design features to use the working fluid. Ceramic bearings $\left(\mathrm{Si}_{3} \mathrm{~N}_{4}\right)$ have been successfully operated in the alternate SSME turbopump turbomachine (Gibson and Fears [1992]) as have the von Pragenau [1992] damping bearings in the space shuttle main engine (SSME) turbomachine tests (McConnaughey et al. [1992]). Magnetic bearings were installed but removed from some compressor applications; efforts to reintroduce magnetic bearings continue at the Electric Power Research Institute, NASA (DiRusso and Brown [1992]), the U.S. Air Force, and elsewhere. For example, Schmied [1991] describes the development of a hermetically sealed pipeline compressor called MIPICO. The compressor consists of a high-speed, gas-cooled electrical drive $(10000 \mathrm{rpm})$ and is supported on magnetic bearings with corrugated ribbon-damped backup roller bearings.

Magnetic bearings promise very low losses, long life, and no lubrication, but at an increase in control complexity, and currently have load capacity and weight limitations. Foil bearings hảve been successfully operated in air-cycle machines for aircraft for years (Emerson [1978]) and have recently been successfully tested in the cryogenic fluids hydrogen (Gilbrech et al. [1993]) and oxygen (Genge [1993]). Combinations of these bearings have been proposed, but none are now in service. 
Conventional fluid film bearings have been pressed into service in the interim.

Seals function as the interface between the power stream (main engine flow) and the environment or the secondary flow systems of the turbomachine. A variety of seals, such as labyrinth, face, aspirating, brush, and honeycomb, are available to the designer and are described in various detail in the Seals Code Development Workshop Series (Liang and Hendricks [1991, 1992, 1993]). Generally, interactions between the power stream and secondary flow system components, such as seals, are introduced as fixed points in the engine cycle model, although efforts are being made to maintain salient details within the engine model (e.g., Numerical Propulsion System Simulation, Claus et al. [1991]).

A major effort is being made to enhance the efficiency of turbomachines by integrating those systems with the most impact on operating cost reduction and the least cost of implementation into next-generation engines. Most original equipment manufacturers agree that engine sealing and secondary flow system management have the highest potential. Currently, the need is to develop a set of tools to interactively couple the flow and dynamic characteristics of the seals, the cavities, and the power stream while introducing new concepts and satisfying environmental constraints.

In this paper we draw heavily upon work at NASA Lewis Research Center for background material and explore the development of

1. Thermophysical property codes (required by design and research activities)

2. Hydrostatic and hydrodynamic effects in bearings (conditions where hydrostatics emerges as the support effect and those where the hydrodynamics dominate, as well as pocket flow details)

3. Damping seals and bearings and the dual-function component termed "damping seal/bearing" (controls leakage, supports rotor loads, and significantly enhances turbomachine stability)

4. Combined effects of hydrodynamic and foil interactions in foil bearings (foil flexure "bumps" provide support and dynamic stability; work well at high speed)

5. Labyrinth and brush seal concepts and seal impact on engine efficiency (brush seals outperform labyrinth seals but changeout life has not been established)

6. Interactive coupling of power stream (main flow) with secondary airflow systems (flows over the blade tips, vortex control, cavity flow, and power stream) for both rocket and jet engine turbomachines.

\section{THERMOPHYSICAL PROPERTIES OF FLUIDS}

At increased fluid pressures and densities thermophysical property variations are important. Many designers have limited access to thermophysical properties codes, such as the NASA codes GASP (Hendricks et al. [1975]), WASP (Hendricks et al. [1973]), FLUID (Fessler[1977]), GASPLUS (Fowler [1987]), complex chemical equilibrium (Gordon and McBride [1976]), gaseous hydrocarbons \{Johnson [1971]), and National Institute for Science and Technology (NIST, formerly NBS)-sponsored equation-of-state codes MIPROPS (Brennan et al. [1992]) and ALLPROPS (Jacobson et al. [1991]), and for fluid mixtures (e.g., Mollerup [1975]). GASP uses an equation of state to provide thermophysical properties of 10 fluids, parahydrogen, helium, neon, nitrogen, oxygen, methane, carbon dioxide, carbon monoxide, fluorine, and argon, from the triple point to elevated pressures and temperatures, including derivatives of thermodynamic functions and saturated properties. WASP provides similar properties for water and steam. FLUID represents a van der Waals-based interpolation scheme for providing table lookup of thermodynamic properties generated from data or from developed programs such as GASP. GASPLUS, an expanded version of GASP, includes GASPAI for fluid air and calculates properties of some fluid mixtures. MIPROPS is similar to GASP but has a higher order equation of state; ALLPROPS is a fundamental Helmholtz free-energy form similar to WASP with a large number of fluids available. The Gordon-McBride codes calculate the complex chemical equilibrium properties of a vast array of gases, including metallic additives for combustion and related processes in the internal combustion engine, jet engines, and rocket engines. The Johnson code provides mixtures of gaseous hydrocarbons for rapid analyses, notably for low-hydrocarbon fuels. The Mollerup code (extended corresponding states) provides properties of fluid mixtures including complex behavior of the dew and bubble locus.

The principles of similarity for thermophysical properties and fluid flow mechanics are discussed by Hendricks and Sengers [1980].

\section{FLUID FILM BEARINGS}

A review of the literature and practical design methodologies for contact bearings is presented in a text edited by E.V. Zaretsky [1992]. Over the years the $D N$ (bearing bore in millimeters times bearing speed in rpm) limitations of contact bearings have edged upward, but fluid 
film bearings (e.g., hydrodynamic, hydrostatic, and foil) have emerged for high- $D N$ applications. A companion book (Zaretsky [1994]), presents a design approach to fluid film bearings. For specific details of analysis and experiment, see, for example, Pinkus [1990], Khonsari [1987], Dowson et al. [1966-67], Hamrock [1991], San Andres [1992, 1993], Braun et al. [1984-85, 1987a,b], Tam et al. [1988], and Petchenev et al. [1992]. We apologize to the many other excellent researchers not cited.

Dynamics are a major part of any turbomachine analysis; details of their origin and seal/bearing behavior are found in a series of NASA Conference Publications on Rotordynamic Instability Problems in High Perfor- mance Turbomachinery (Childs et al. [1980, 1982, 1984, 1986, 1988, 1990, 1993]; see also Childs and Hale [1993], Tam et al. [1988], and Bentley et al. [1986]). In this section our remarks are limited to hydrostatic pocket bearings, damper bearings, wave bearings, and foil bearings.

\section{Hydrostatic Pocket Bearings}

In the hydrostatic pocket bearing, pressurized fluid is fed through a set of orifices and pockets to establish and maintain a lubricant fluid film between the load-bearing rotor and the stator (Fig. 1). The basic principles are easy enough; it is the details of solving the conversation

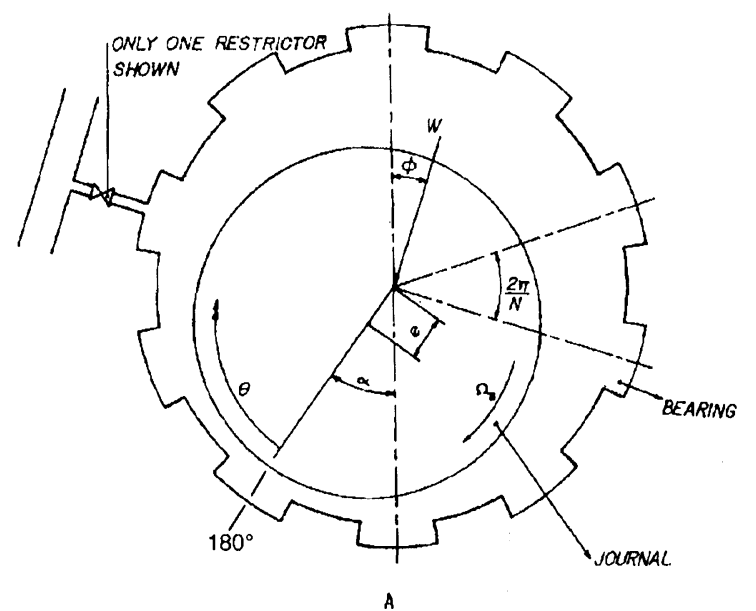

UNWRAPPED BEARING
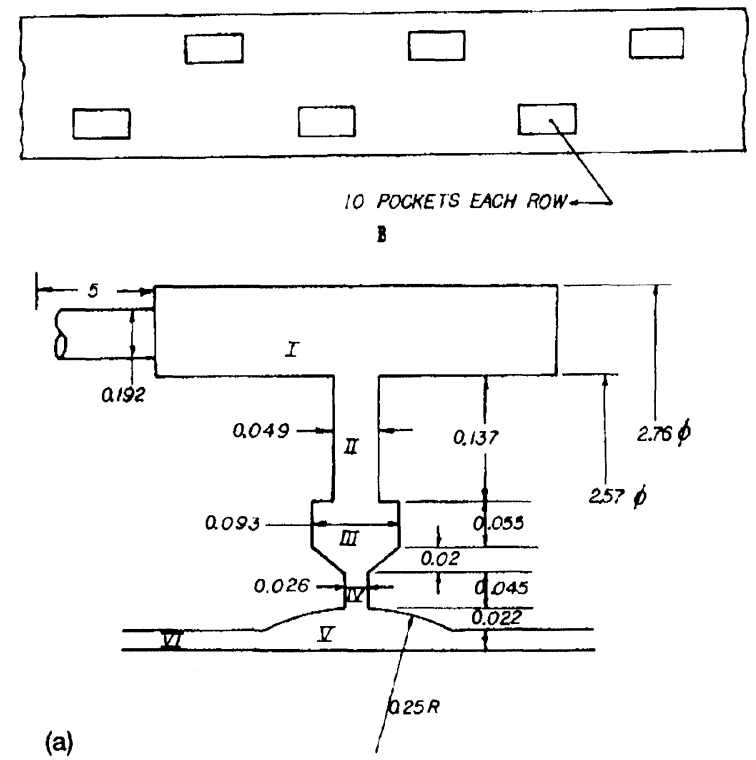
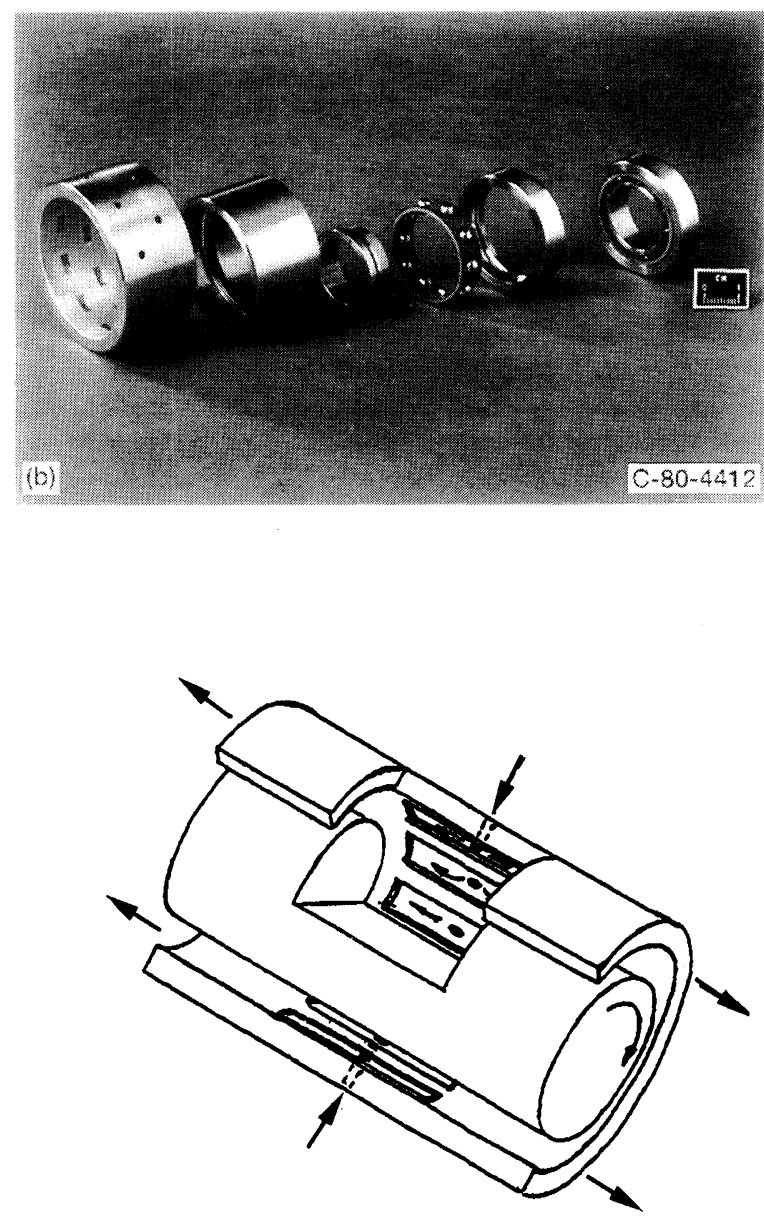

(c)

FIGURE 1 Hydrostatic bearing. (a) Pockets and restrictor. (All dimensions are in inches.) (b) Hybrid hydrostatic test bearing. (c) Sketch. 


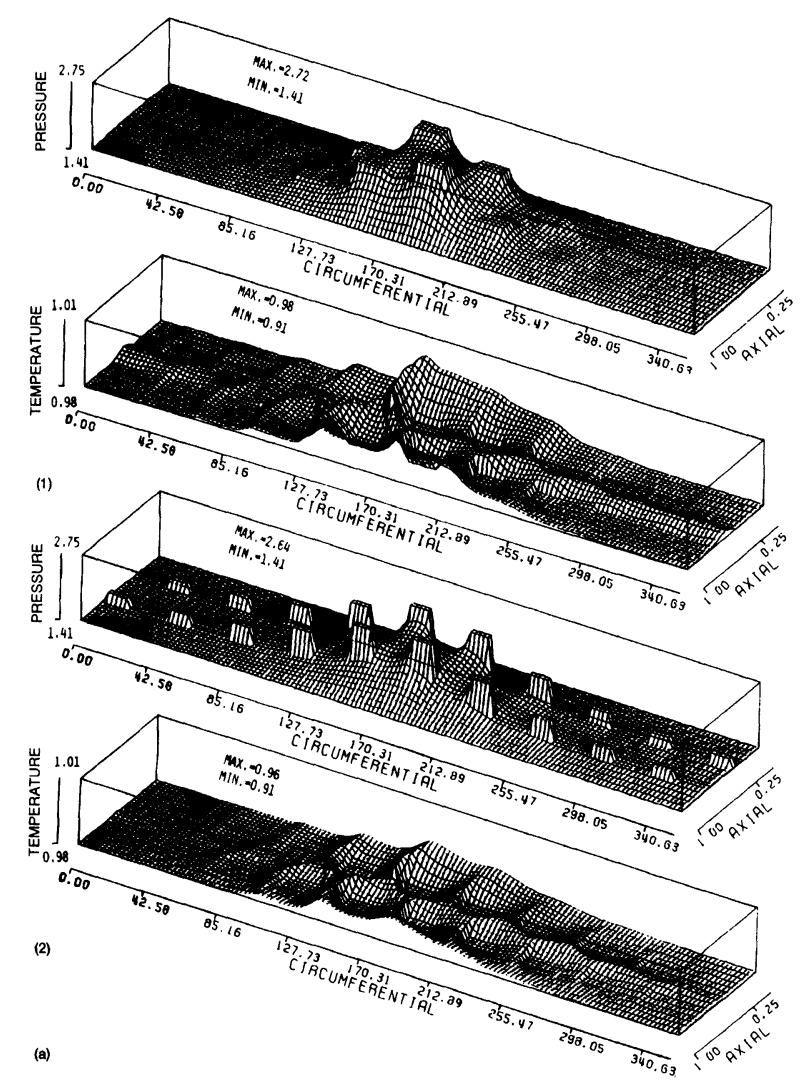

FIGURE 2 Qualitative evaluation of pressure and temperature profiles for variable-property, laminar-flow hydrogen. Eccentricity, 0.7; shaft speed, $100 \mathrm{krpm}$. From Braun et al. [1987a]. (a) (1) Without inertia, (2) with inertia.

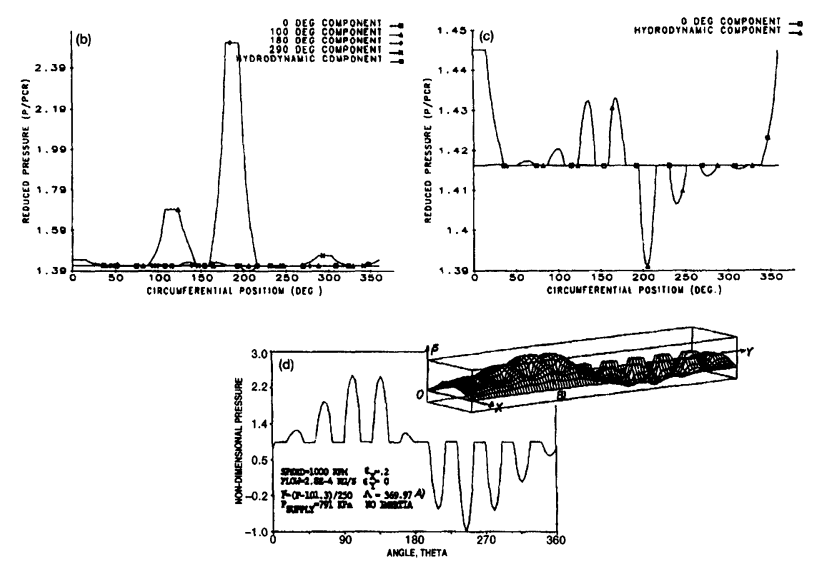

FIGURE 2 Concluded. (b) Comparison between hydrodynamic and four hydrostatic components. (c) Comparison between smallest hydrostatic component and hydrodynamic component. (d) Equivalent hydrostatic oil bearing rotating at $1000 \mathrm{rpm}$. (Cross section through back row and three-dimensional unwrapped pressure profile.) equations (mass, energy, momentum, and concentration) for the film subject to the flows through the pockets that is difficult. Codes such as HYDROS (Braun et al. [1994]) are available for laminar flow solutions of the Reynolds equation for $N$ pockets, and a bulk flow model code by San Andres [1993] is available for turbulent flows. For a complete numerical analysis, including concentration, the code SCISEAL (see sect. 5.1.2) can be adapted.

Global field effects. Figure 2 represents the pressure and temperature maps normalized by the thermodynamic critical points $P_{c r}$ and $T_{c r}$ for a 20-pocket hydrostatic bearing. Figure 2(a)-1 omit convective terms; figures 2(a)-2 include convective terms (Braun et al. [1987b]), but the flow was assumed to be laminar. For high surface speeds or low-viscosity fluids the hydrostatic component is weak relative to the hydrodynamic component (Figs. 2(b) and (c)). Figure 2(d) represents an oil hydrostatic bearing; here the hydrostatic component is larger than the hydrodynamic component, but the results are for laminar flows. Hannum and Nielson [1983] obtained data for a 10-staggered-pocket hydro-static hybrid ball bearing (Fig. 1(b)) in a proof-of-concept test; dynamic test results are provided by Childs and Hale (1993).

Local pocket details. The restrictors control fluid flow into the pockets. The pressurized pockets in turn distribute the fluid to the lands, where the film is formed that supports the bearing load. Until now the behavior of the pocket flows has been modeled as a simple flow source without considering the complexity and interaction between the main-stream flow and the injected flow. There also exists a major difference between shallow- and deep-pocket flow behaviors, both of which are strongly coupled to the main flow. The effects of the pocket geometry (e.g., square, ramped Rayleigh, circular) are discussed by Braun et al. [1994].

Shallow-pocket flow: The calculated streamlines for a shallow pocket are illustrated in Figure 3. Note the

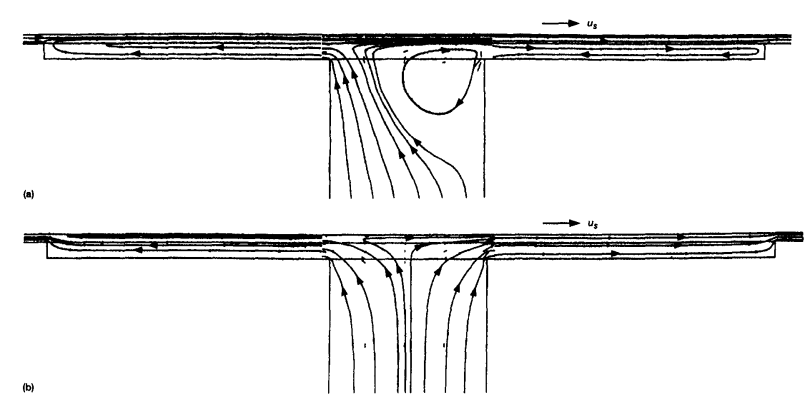

FIGURE 3 Direct-pocket-feed hydrostatic bearing streamlines at two injection-to-surface-flow ratios $\left(f_{j} / u_{s}\right)$ with clearance of $0.5 \leq$ pocket depth. (a) $f_{j} / u_{s}=$ (Couette dominated). (b) $f_{j} / u_{s}=4$ (jet dominated). 
circulation patterns and the load-carrying pinch point (right-hand portion of fig. 3 ) where the pressure tends to spike owing to the Rayleigh step effect. The major differences are (1) the load-carrying "pillow" formations and (2) the development of a recirculation "bubble" within the "orifice" restrictor jet tube (Fig. 3(a)). For Couette-dominated flows the injection-to-surface-speed parameter ratio $f_{j} / u_{s}$ is small (Fig. 3(a)). Increasing the restrictor pressure drop increases $f_{j}$ (jet-dominated flow); the "bubble" essentially vanishes (Fig. 3(b)) and the pressure spike at the pinch point vanishes. The pressure becomes more evenly distributed, with a maximum near the jet centerline. For jet-dominated flows convective effects are noted on each side of the pocket, but for Couette-dominated flows the convective effects are concentrated in the Rayleigh step.

Although not calculated herein, the dynamics of such strongly coupled flow systems will significantly affect the rotor/bearing dynamics. The contrasting flow behavior and the influence of shear-driven flow in the shallow pockets provide new insights into hydrostatic bearing developments.

Deep-pocket flow: Comparing the calculated streamline for deep-pocket flows (Fig. 4) with that for shallow-pocket flows (Fig. 3) shows an advance of the

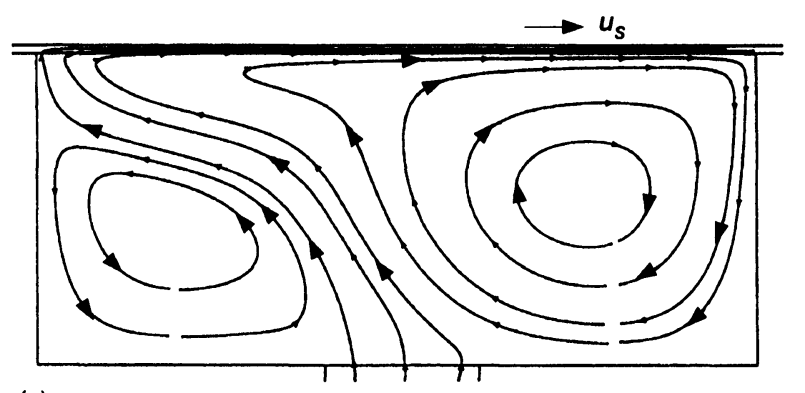

(a)

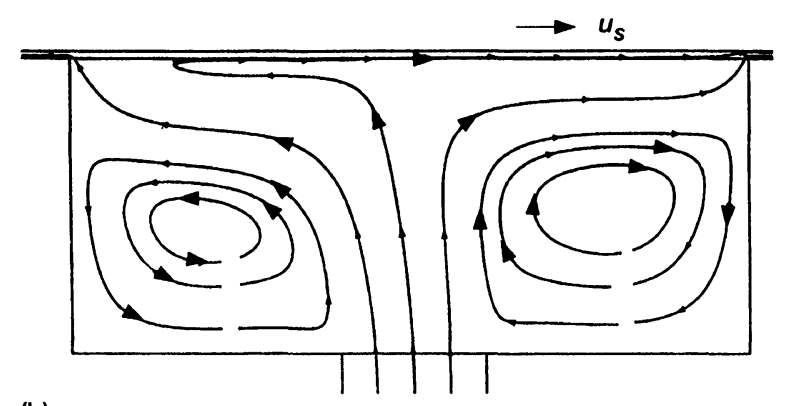

(b)

FIGURE 4 Deep-pocket-feed hydrostatic bearing streamlines at two injection-to-surface-flow ratios $\left(f_{j} / u_{s}\right)$. (a) $f_{j} / u_{s}=$ (Couette dominated). (b) $f_{j} / u_{s}=4$ (jet dominated). recirculation "bubble" and Rayleigh step effects into the deep cavity, engendering two distinct vortices. The deep-pocket flow circulates about these double vortices within the cavity before entering the flow field near the Rayleigh step (right side of Fig. 4). In figure 4(a) $f_{j} / u_{s}$ is small and the flow is Couette dominated; Figure 4(b) represents jet-dominated flow. In either case the double vortices remain, but their location and the carryover into the upstream flow is noted (left pocket edge, Fig. 4(b)). (Continuity considerations in both hydrodynamic and hydrostatic pockets lead to flow reversals; the hydrodynamics are shear driven and the hydrostatic are pressure driven.)

Qualitative comparison: Figure 5 provides a qualitative comparison of calculated and experimental pocket flows. The computational grid, the flow streamlines, and the streak lines are shown in Figures 5(a), (b), and (c), respectively. The correspondence between calculated and observed flow patterns is quite remarkable.

The hydrostatic bearing represents one of four strongly coupled main-stream and secondary flow systems to be addressed herein.

\section{Fluid Film Damping Seal/Bearing}

Conventional wisdom is that seals inhibit leakage and bearings carry loads; however, an eccentric seal becomes a lightly loaded bearing and can enhance or degrade turbomachine stability. The von Pragenau [1982, 1985] fluid film damping seal/bearing combines these functions into a single mechanical component and represents a major innovation.

Damping seal. It is well established that seals (e.g., labyrinth seals) restrict leakage through the annular gaps of shafts (e.g., in turbomachines). The seals are also lateral bumpers that contain the damage from whirl. The whirl forces from labyrinth seal and turbine blade tip leakages and internal rotor friction destabilize a rotor operating above the critical speed with rolling-element bearings (which lack damping). Speed limits can conflict with the power requirements, as experienced on the cryogenic high-pressure turbopumps of the SSME. The whirl problem prompted the invention of damping seals (von Pragenau [1982]), with successful flight hardware testing in 1985 (von Pragenau [1985]), and experimental studies by Iwatsubo and Sheng [1990].

The damping seal consists of a pocketed stator (Fig. 6(a)) and a smooth journal. The seal is not a hydrodynamic bearing, since axial flow controls the pressure distribution in the annular seal gap. The isogrid pattern of Figure 6(a) is ideal for high flow resistance, but other patterns can be selected for easier manufacturing. Damp- 

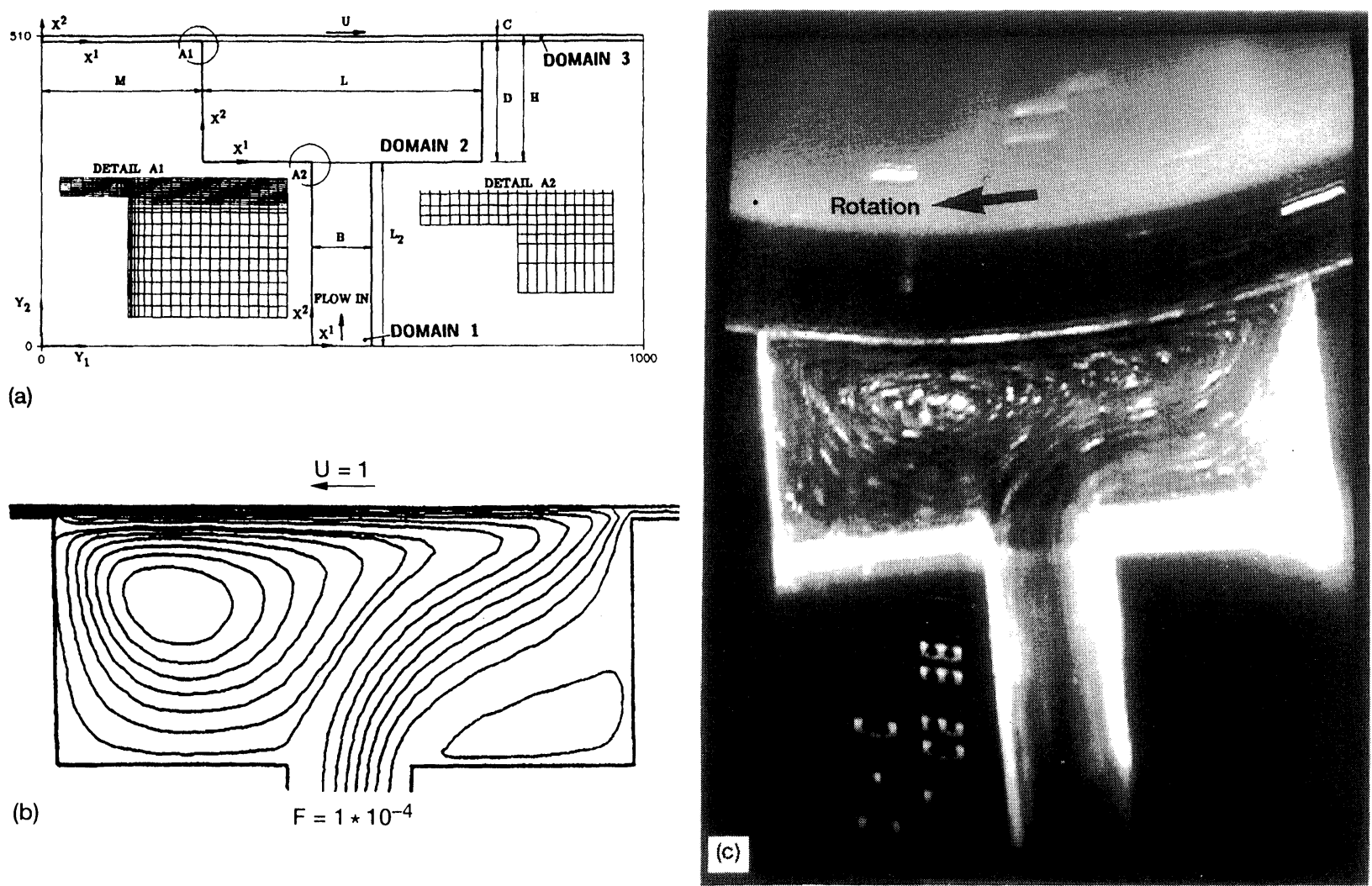

FIGURE 5 Qualitative comparison of calculated and experimental deep-pocket-feed hydrostatic bearing. (a) Computational grid and geometry. (b) Flow streamlines $\left(\mathrm{U}=1, \mathrm{~F}=10^{-4}\right)$. (c) Experimental flow streamlines.

ing seals are comparable in leakage, size, and operation to labyrinth seals. The latter have deep circumferential grooves that can be readily cut on a rotor but are dynamically and stress-wise better in a stator. Constructing a damping seal is like filling the stator grooves of a
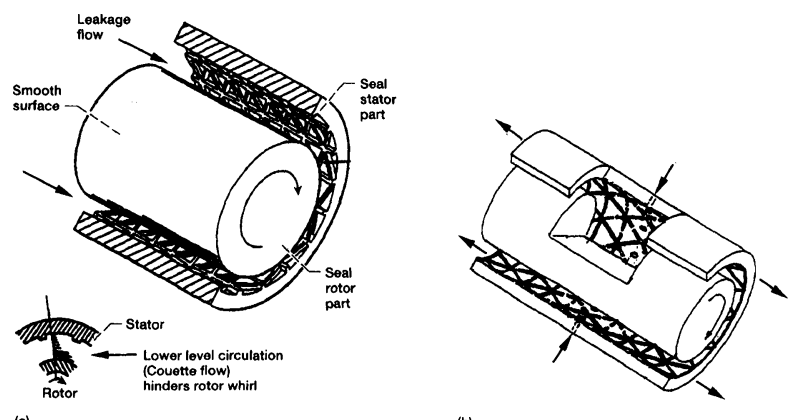

FIGURE 6 Damping seals and bearings. (a) Damping seal concept with isogrid stator. (b) Damping bearing concept with stationary orifices (orifices can also be in shaft). labyrinth seal with many circumferential partitions to inhibit the equalization of diametric pressure differentials.

In a damping seal the rotational flow or average circumferential velocity ratio $\left\langle V_{\theta}>/ R \omega=\lambda\right.$, from the combined effects of rotor drag and pressure drop, is much reduced. For smooth seals and bearings $\lambda \approx 0.43$; for damping seals $\lambda \approx 0.2$ and is even smaller for highly turbulent flows. Generally, a smaller $\lambda$ implies enhanced stability (i.e., the speed limit varies as the first critical speed divided by $\lambda$ (i.e., WOS $\approx \Omega_{c l} / \lambda$ ). The speed limit or whirl onset speed (WOS) is where the rotor starts to orbit unstably at a rotor resonance. The orbits grow until damage occurs or seal rubbing limits the whirl orbit at benign levels. The simple WOS rule is invalid if all damping seals attract nodal points of the first rotor mode or if other whirl drivers (e.g., internal rotor friction) dominate. Then the WOS is predicted with complex rotordynamic analyses that include rotor bending modes and distributed rotor support points of seals, bearings, and whirl drivers. 
TABLE I

Generic Bearing Comparison

\begin{tabular}{|c|c|c|c|c|}
\hline \multirow[t]{2}{*}{ Property } & \multicolumn{4}{|c|}{ Bearing type } \\
\hline & Ball & Roller & Hydrostatic & Damping \\
\hline Moving part count & 33, Duplex & 17 & 1 & 1 \\
\hline$D N$, bore $\mathrm{mm} \times \mathrm{rpm}$ & $<2 \times 10^{6}$ & $<2 \times 10^{6}$ & $\mathrm{n} / \mathrm{a}$ & $\mathrm{n} / \mathrm{a}$ \\
\hline Hoop stress, MPa (ksi) & $172(25)$ & $310(45)$ & $\mathrm{n} / \mathrm{a}$ & $\mathrm{n} / \mathrm{a}$ \\
\hline Hertzian stress, MPa (ksi) & $2412(350)$ & $2412(350)$ & $41(6)$ & $41(6)$ \\
\hline Precision, $\mu \mathrm{m}(\mathrm{mil})$ & $0.5(0.02)$ & $0.5(0.02)$ & $25(1)$ & $25(1)$ \\
\hline Rolling stability & Skidding & Skewing & $\mathrm{n} / \mathrm{a}$ & $\mathrm{n} / \mathrm{a}$ \\
\hline High side load & Wear & Skewing & ok & ok \\
\hline Whirl onset speed & Limit & Limit & Limit & None \\
\hline Bearing damping & Negligible & None & High & High \\
\hline Bearing tilting & $<1^{\prime}$ & $<<1^{\prime}$ & $>1^{\prime}$ & $>1^{\prime}$ \\
\hline Deadband/fitting & Conflict & Conflict & Relaxed & Relaxed \\
\hline Failure mode & Catastropic & Catastropic & Benign & Benign \\
\hline Bearing life limit & Wear & Skewing & None $^{a}$ & None $^{\mathrm{a}}$ \\
\hline
\end{tabular}

${ }^{a}$ No wear at full speed. Only light brusing for a few revolutions at start and stop.

Damping bearing. Damping seals can be considered as half damping bearings, since they are fed from one end through an annular gap like an orifice. The simplest form of damping bearing is a duplex damping seal fed from the middle through an annular recess. Stiffness and damping are increased by feeding a partitioned annular recess in the middle through several orifices, which can even rotate (Fig. 6(b)). The orifices drop the pressure to half the available source pressure to maximize stiffness, as in hydrostatic bearings. Hydrostatic bearings (Fig. 1) have orifices in large recesses that feed the surrounding smooth lands. The narrow gaps between the lands and the rotor affect the flow from the orifices, which produce stiffness as in damping bearings. The damping bearings compare as follows: pocket groups (small pockets joined through gates in the pocket walls) replace the large hydrostatic pocket recesses by surface bearing area; orifices can also rotate with the shaft; and small pockets cover the large land area. Operationally, damping bearings have low leakage, low flow rotation, high stiffness, low whirl forces, high damping, and an insensitivity to rubbing (see Tables I and II).

Damping seal/bearing. The leakage and load capacity of several damping seal/bearings are given in Table I. By design, the clearances, length, and grid are controlled to minimize the leakage-to-load-capacity ratio. To determine the fluid film effects, Lucite damping seal/bearings have been fabricated (Fig. 7) for testing in a fluid-driven turbomachine. No data are yet available.

\section{Wave Bearing}

Figure 8(a) illustrates a three-wave, waved journal bearing with a wave amplitude $e_{w}=0.5 c$ (typically $0.2<\epsilon_{w}$

TABLE II

Dynamic and Leakage Data of Seals and Bearings

\begin{tabular}{|c|c|c|c|c|c|c|c|}
\hline \multirow[t]{2}{*}{ Seal or bearing } & \multicolumn{2}{|c|}{ Stiffness } & \multicolumn{2}{|c|}{ Damping } & \multirow{2}{*}{$\begin{array}{l}\text { Whirl } \\
\text { frequency } \\
\text { ratio }\end{array}$} & \multicolumn{2}{|c|}{ Leakage } \\
\hline & $\mathrm{MN} / \mathrm{m}$ & $\mathrm{klb} / \mathrm{in}$. & $\mathrm{kN}-\mathrm{s} / \mathrm{m}$ & lb-s/in. & & $\mathrm{kg} / \mathrm{s}$ & $\mathrm{lb} / \mathrm{s}$ \\
\hline HPOTP duplex ball bearing & 70 & 400 & 3.5 & 20 & & ${ }^{\mathrm{a}} 2.7$ & 6 \\
\hline HPOTP pump damping seal & 102 & 580 & 53 & 300 & 0.19 & 2.7 & 6 \\
\hline HPOTP turbine damping bearing & 665 & 3800 & 151 & 860 & .11 & 8.3 & 19 \\
\hline HPOTP turbine hydrostatic bearing & 578 & 3300 & 123 & 700 & .30 & 16 & 35 \\
\hline HPFTP damping seal & 29 & 166 & 39 & 224 & .17 & .4 & .9 \\
\hline HPFTP smooth seal & 76 & 434 & 27 & 154 & .50 & .8 & 1.67 \\
\hline HPFTP turbine damping bearing & 823 & 4700 & 172 & 980 & .09 & 1.5 & 3.3 \\
\hline HPFTP turbine hydrostatic bearing & 771 & 4400 & 112 & 640 & .30 & 2.8 & 6.2 \\
\hline HPFTP turbine roller bearing & 613 & 3500 & $\mathrm{n} / \mathrm{a}$ & $\mathrm{n} / \mathrm{a}$ & & ${ }^{\mathrm{a}} 1.1$ & 2.5 \\
\hline
\end{tabular}

a Leakages through ball and roller bearings are coolant flows. All HPOTP and HPFTP data are for 30000 and 36000 rpm, respectively. 


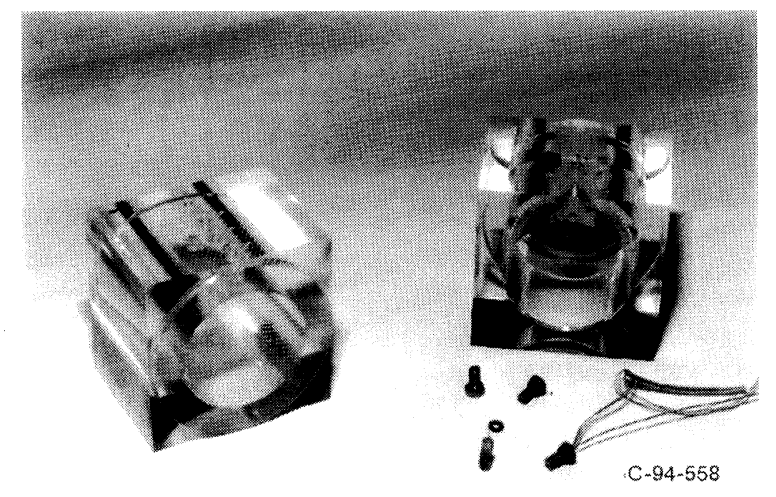

FIGURE 7 Damping seal/bearing diamond knurl patterns and associated pressure probes.

$=e / c<0.6)$. Relative to the circular journal bearing the calculated load capacity is increased 2 to 4 times and dynamic stiffness is increased 4 to 10 times (Dimofte [1993]). The sensitivity of the applied load to the wave position diminishes when more waves are used. In separate testing (Dimofte and Addy [1993]) good agreement was found between measured and calculated midplane pressure distributions (Fig. 8(b)) at $15069 \mathrm{rpm}$, a load of $141 \mathrm{~N}(31.8 \mathrm{lb})$, and a clearance of $19 \mu \mathrm{m}$ (0.00075 in.), or $\epsilon_{w^{\prime}}=0.547$.

\section{Foil Bearings}

Foil bearings represent a relatively new technology (Fig. 9); the understanding of how the film interacts with the foil to support the load remains undeveloped.

Background. About 1952, NACA realized that rolling-element bearings would not satisfy future aerospace requirements and embarked on a foil bearing study led by W.J. Anderson and Z. Nemeth. Nemeth [1994] told of constructing a two-ring device with the attached foil forming a catenary under load. However, the 71-N (16-lb) shaft had to be lifted by a separate air bearing until the shaft rotation achieved $7000 \mathrm{rpm}$, where transition to the foil bearing occurred. Subsequent work by Blok and van Rossum [1953], Eshel and Elrod [1965], Licht and Branger [1973a,b], Heshmat et al. [1983], Heshmat [1993], Saville et al. [1991], Gilbrech et al. [1993], Genge et al. [1993], Carpino [1990], Ping and Carpino [1993], Braun et al. [1994], and others have advanced foil bearings.

Applications. Foil bearings have been used in small, high-speed, lightweight turbomachines ranging from air units, such as superchargers and auxiliary air-handling units (Emerson [1978], O'Connor [1993], Dill and Hesh- mat [1993]), to Brayton cycle systems and cryogenic turbomachines for space power. Space transfer vehicles and other high-speed, lightweight, high-performance propulsion systems that require long life can overcome limitations inherent to conventional bearings (e.g., $D N$ ) through the use of foil bearings.

Application in high-performance cryogenic turbomachines has been demonstrated at NASA Lewis Research Center in liquid hydrogen foil-bearing tests with a measured load capacity of over $1.66 \mathrm{MPa}$ (240 psi) (Saville et al. [1991]). The Teflon-coated foil bearing was essentially wear free after more than 130 start-stop cycles and had good dynamic stability to $97000 \mathrm{rpm}$ for a 44.4-mm- (1.75-in.)-diameter rotor (surface speed of
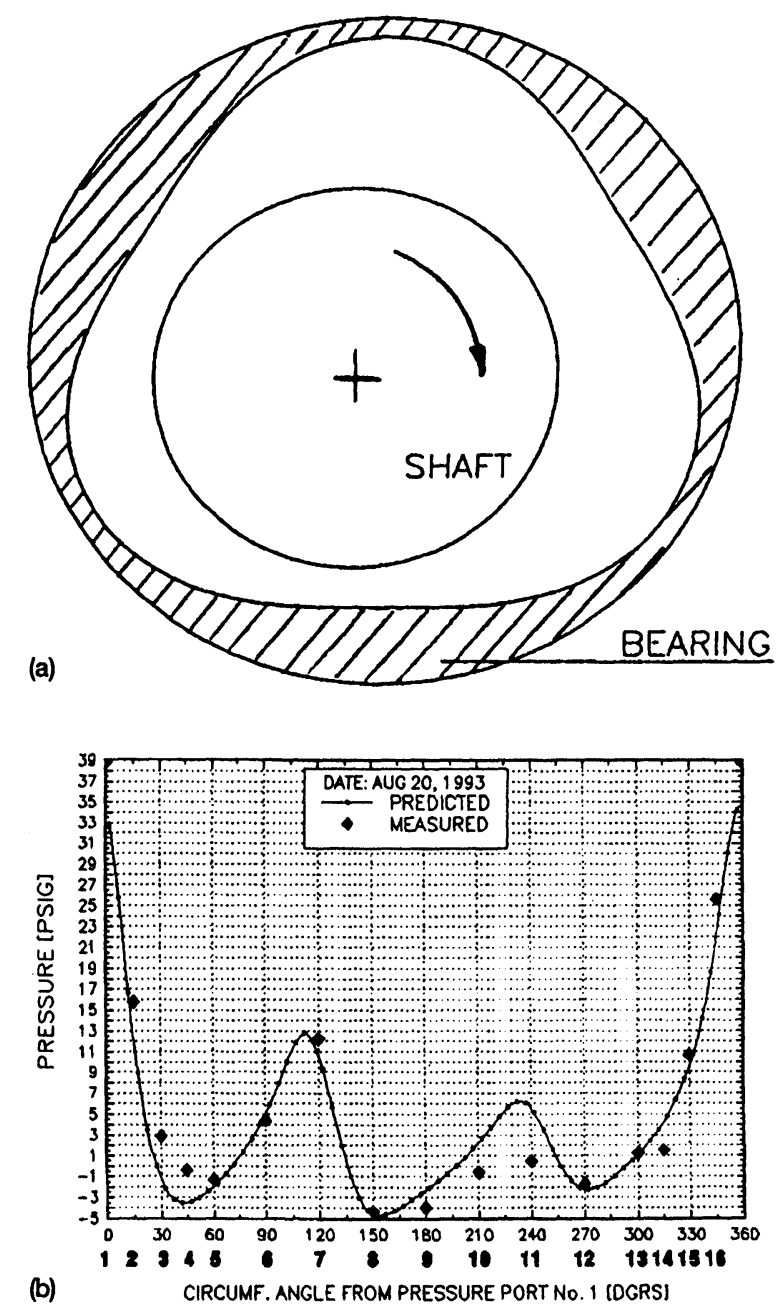

FIGURE 8 Waved journal bearings. (a) Concept (wave height and clearance greatly exaggerated). (b) Predicted and measured pressures in bearing middle plane at $15069 \mathrm{rpm}$; load, $31.8 \mathrm{lb}$; clearance, $19.0 \mu \mathrm{m}$. 

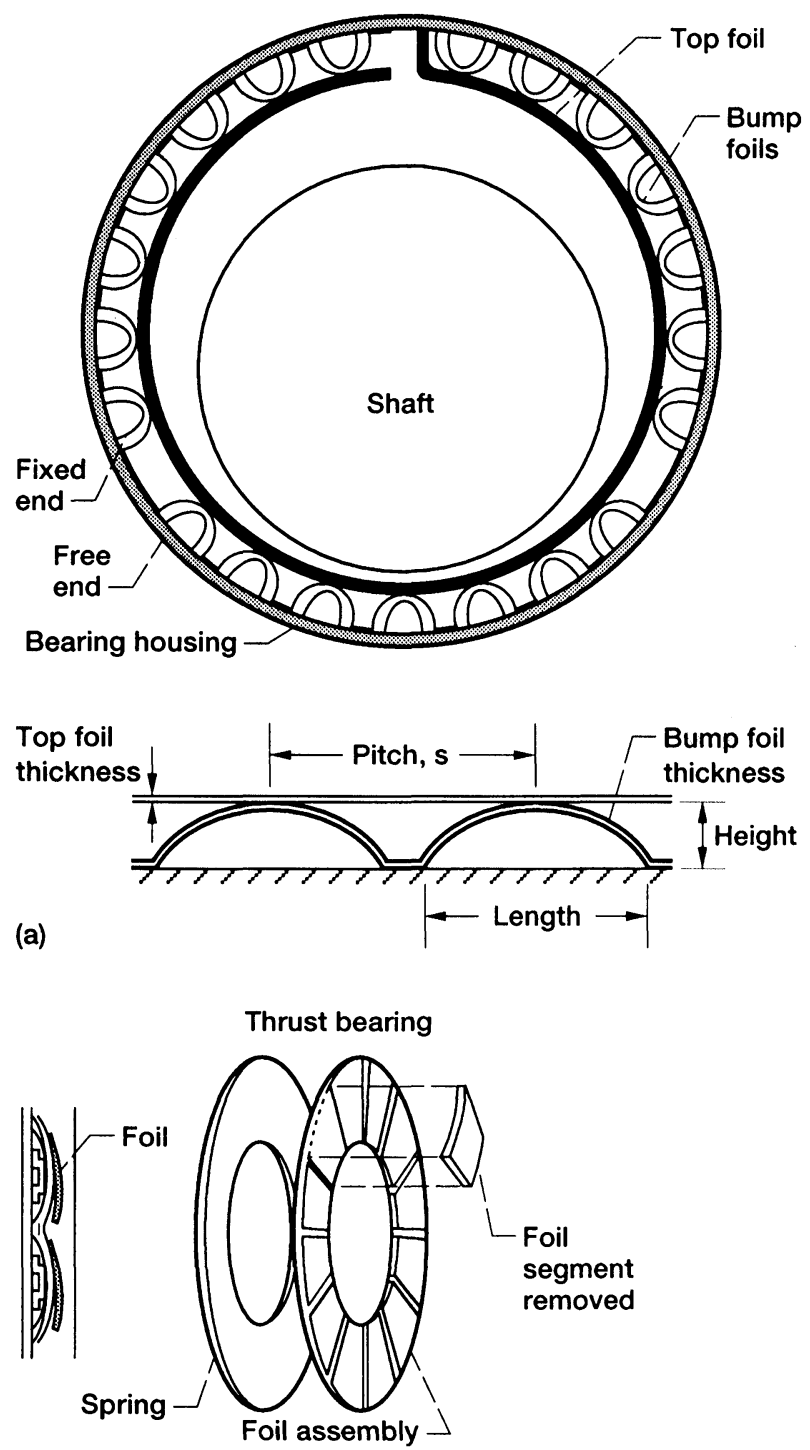

(c)
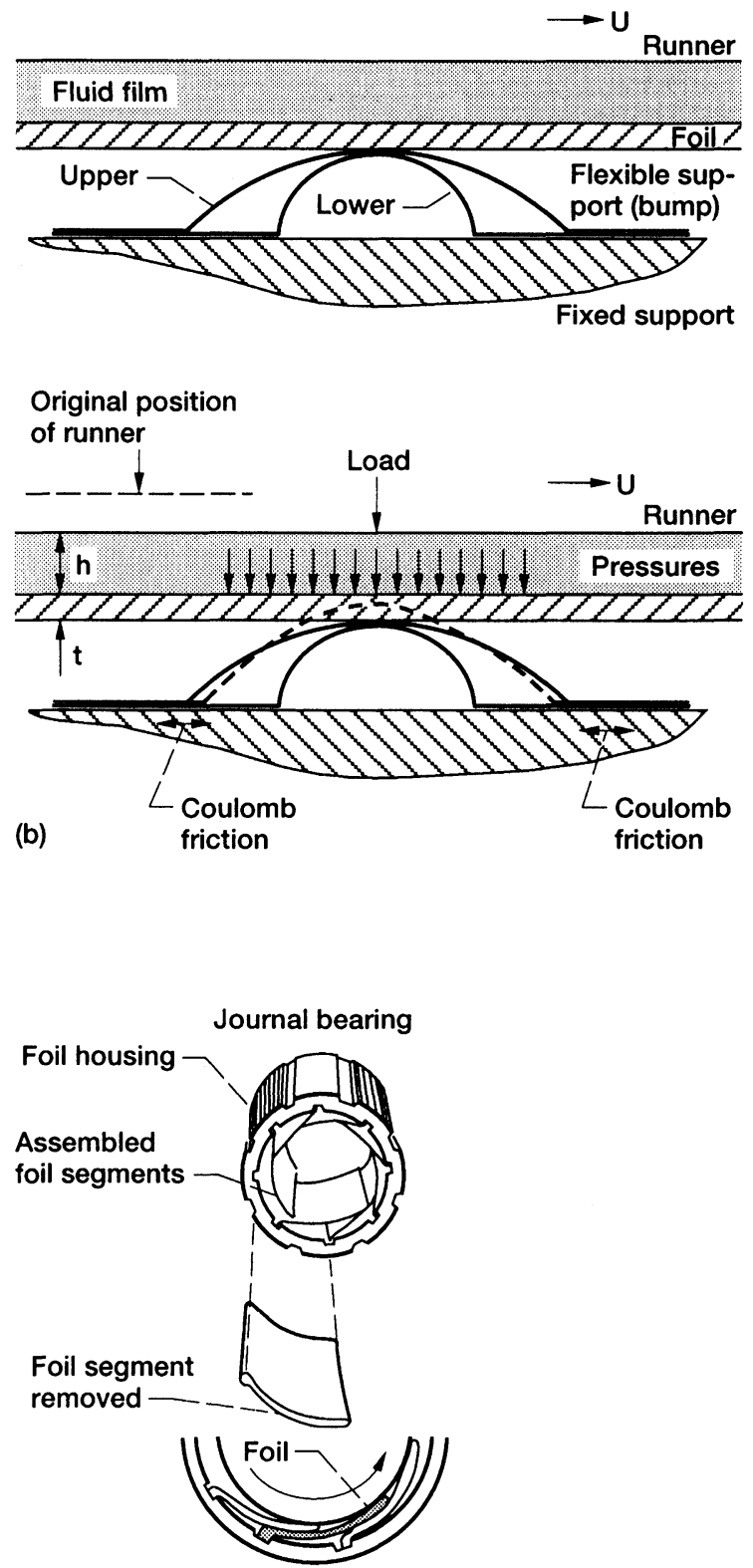

FIGURE 9 Fluid film foil bearings. From O'Connor [1993]. (a) Bump-foil journal bearing concept. (b) Schematic of bump-foil loading. (c) Leaf-foil face and journal bearing concept.

$226 \mathrm{~m} / \mathrm{s}(740 \mathrm{ft} / \mathrm{s}))$. A similar foil-bearing test (Genge et al. [1993]) generated more than $1.83 \mathrm{MPa}$ (266 psi) in liquid oxygen (Fig. 10(a)) and $2.42 \mathrm{MPa}$ (350 psi) in liquid nitrogen (Fig. 10(b)). After more than 120 startstop cycles (100 min of operation) with acceptable stiffness, damping coefficients up to 7 to $8.8 \mathrm{~N}$-s/mm (40 to $50 \mathrm{lb}$-s/in.) were measured. No subsynchronous whirl was noted throughout all tests. Power loss was estimated to be less than 1 percent of turbomachine power.
Tests at NASA Stennis Space Center of an Allied Signal/McDonnell Douglas subscale liquid hydrogen turbopump (Gilbrech et al. [1993]) demonstrated feasibility at speeds to $91000 \mathrm{rpm}$ (about 244-m/s (800-ft/s) surface speed). The machine achieved discharge pressures to $6.9 \mathrm{MPa}(1000 \mathrm{psi})$, started without total precool (chilldown), and had bearing coolant flow less than 1 percent of total flow. The turbopump was tested for more than 100 start-stop cycles (run time of $50 \mathrm{~min}$ ) with no 

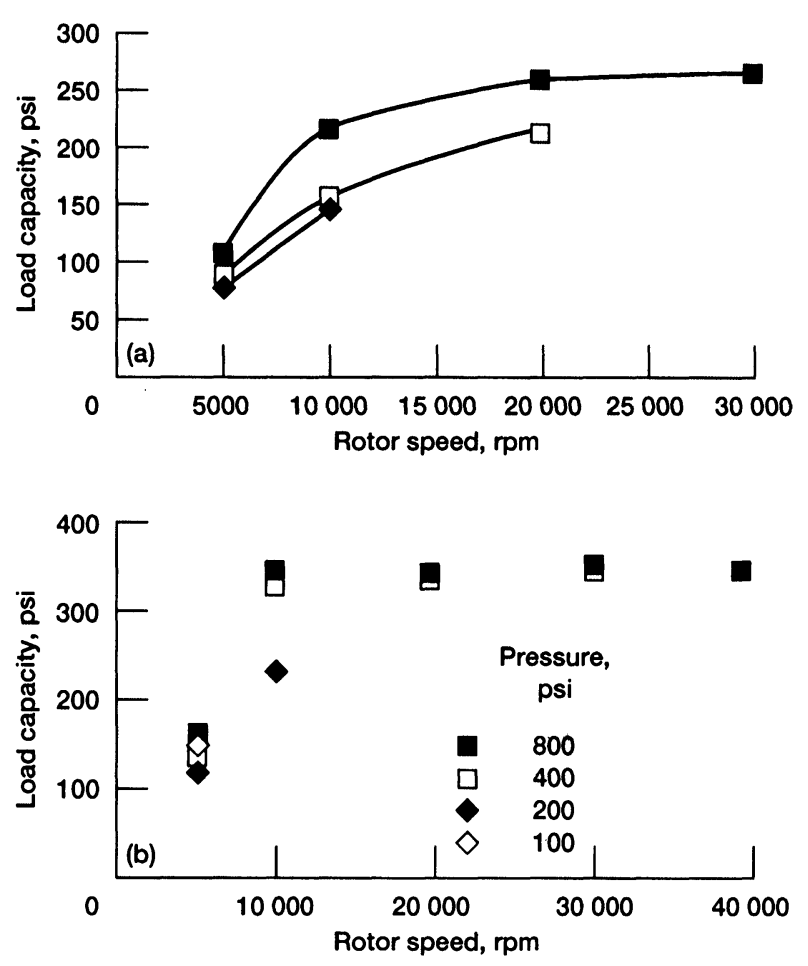

FIGURE 10 Turbomachine load tests of cryogenic foil bearing. From Genge et al. [1993]. (a) Liquid oxygen. (b) Liquid nitrogen.

head loss when inlet pressure was reduced from 0.28 to $0.17 \mathrm{MPa}$ (40 to $25 \mathrm{psi}$ ). The pump exhibited stable operation with only $0.013-\mathrm{mm}(0.5$-mil) excursions at 60 $000 \mathrm{rpm}$ and 0.05 -mm (2-mil) excursions under cavitation conditions. The foil bearings were reported to be in excellent condition. These and other tests developing the limitations and advantages of foil bearings are cited in Table III, with some applications cited in Table IV.

It is evident that a direct comparison between competing bearings (roller, ball, ceramic, wave, foil, magnetic, hydrostatic, hydrodynamic, and varieties of hybrids) is required. Comparisons are generally made on a point design basis and not in the comprehensive fashion warranted by the technology.

Principles and construction. The principles of a foil bearing are simple: a high-speed shaft rests on a fluid film wedge like a cushion supported by an assembly of curved leaf springs. Two types of curved springs are (1) convergent foils set on multiple-stiffness "bump" pads and (2) overlapping leaf springs (Fig. 9), (Heshmat et al. [1983], Heshmat [1993], Saville et al. [1991], and O'Connor [1993]). Foil bearings are compliant and are dynamically stable (Fig. 3). At high speeds (Fig. 4) the bearings can carry high loads such as the $0.69 \mathrm{MPa}(100$ psi) at 4.2 million $D N$ demonstrated by Heshmat [1993] and Dill and Heshmat [1993]. Foil bearings will rub at low speeds and have significant runout at high loads. The bearing materials used are Inconel, beryllium copper, and various stainless steels. Under consideration are superalloys such as MA-2000 for temperature applications as high as $1095^{\circ} \mathrm{C}\left(2000^{\circ} \mathrm{F}\right)$. Solid lubricant coatings and other coatings enhance foil life through tribological pairing.

The foil thicknesses vary from 0.1 to $0.28 \mathrm{~mm}(0.004$ to 0.011 in.) depending on the bearing and the fit cartridge style into the bearing. The clearance-to-radius ratio is usually $5 \leq 10^{-3}$ to $10^{-2}$ and can be nearly twice that of conventional journal bearings with film thicknesses of 100 to $200 \mu \mathrm{m}$ ( 0.004 to $0.008 \mathrm{in}$.). The stiffness is a combination of that generated by the convergent fluid film wedge (O'Conner [1993], Heshmat et al. [1983], and Heshmat [1993]) and the mechanical design of the leaves and "bumps," which are separated by 0.025 to $0.076 \mathrm{~mm}(0.001$ to $0.003 \mathrm{in}$.) to provide a nonlinear stiffness. Damping is engendered both by the fluid film and the friction characteristics of the mechanical foil or the foil and bumps depending on the class of design.

It is evident that foil bearings are compliant and stable in compression (but can be unstable in tension) and support significant loads, although understanding of the interaction between the fluid film developed on the compliant foil, the feed stream, and the load is undeveloped.

Foil modeling. Consider the physics: in the foil bearing the fluid film thickness is large and the "area within the contact" is large; whereas in the fixedgeometry fluid film or rolling-element bearing the fluid film is very thin and the "area within the contact" is very small. Such are the characteristic differences between fixed geometries (e.g., journal bearings) and complaint bearings (e.g., foil bearings).

The early work of Blok and van Rossum [1953] provides insight into development of a nominally constant-thickness film and the foil load capacity and compares friction factors between a foil bearing and a journal bearing (Fig. 11). The viscous resistance-to-load-capacity parameter $\mu N / p_{m}$ is dimensionless, where $\mu$ is the viscosity, $N$ the rotor speed, and $p_{m}$ the specific pressure $\left(p_{m}=\right.$ foil tension/radius of curvature). At high rotor speeds the foil bearing has less friction than the classic bearing but higher torque at high loads for the simple tensile foil used in the experiment. Heshmat's [1983, 1993] detailed calculations of the bump-foil concept (Fig. 9(c)) accounted for foil deflection and bump-foil deflection with foil friction to determine the static and dynamic load capacity. The film thickness is not quite constant; minimums in the nominal film thickness appear (Fig. 12) that increase at low foil stiffness. Carpino 
TABLE III

Advantages and Disadvantages of Foil Bearings

\begin{tabular}{|c|c|}
\hline Test & Advantage \\
\hline Accommodations & $\begin{array}{l}\text { Accommodates distortions and misalignments. } \\
\text { No strict tolerances on journal size or roundness because foil self-tension conforms. } \\
\text { Because both journal and foil can deflect, can accommodate relatively large excursions without } \\
\text { destroying the fluid film. } \\
\text { Can operate at elevated temperatures in the presence of appreciable temperature gradients (not } \\
\text { subject to thermal distortion). } \\
\text { Can operate at over } 5 \text { million } D N \text { (upper } D N \text { limits have not been established). }\end{array}$ \\
\hline Stability & $\begin{array}{l}\text { Self-excited vibrations do not occur; deflections of foils tolerate excursions and misalignments; } \\
\text { stiffness and damping can be maintained by foil selection. } \\
\text { High-speed performance and rotordynamics insensitive to numerous start-stop cycles. Resistant } \\
\text { to high-speed rubs. }\end{array}$ \\
\hline Low wear & $\begin{array}{l}\text { Excellent wipe-wear, start-stop, and longevity characteristics. } \\
\text { The foil is forgiving and wear is benign. } \\
\text { No external pressure source is required for starting or stopping. } \\
\text { Long life is anticipated. }\end{array}$ \\
\hline Foreign object damage & $\begin{array}{l}\text { Large film thickness and foil conformability provide relative immunity from abrasive particulates } \\
\text { and foreign matter entering the bearing clearance. } \\
\text { Reasonable extension to liquids, especially with particle-laden lubricants and in machines using } \\
\text { contaminated process fluids as lubricants. } \\
\text { Simple basic construction and extremely wide tolerance of nominal journals dimensions. }\end{array}$ \\
\hline High capacity and low torque & $\begin{array}{l}\text { High load capacity-thicker films carry heavier loads than conventional-film-thickness bearings } \\
\text { with sensibly low starting torques. } \\
\text { Lower power loss-large films imply lower power losses, typically less than } 1 \text { percent of turbine } \\
\text { power loss. } \\
\text { Surface taper, microgrooving, or Rayleigh surfaces enhance low-speed liftoff. }\end{array}$ \\
\hline Small weight and envelope & Lighter weight and smaller space envelope than conventional bearings. \\
\hline Wear & $\begin{array}{l}\text { Wear of supporting foil can lead to catastrophic fracture. } \\
\text { Rubbing motion of "bumps" and damping is not understood. } \\
\text { Starting-stopping friction wear could be large. }\end{array}$ \\
\hline Design & Few analytical tools or design charts are available; design is by perturbation method. \\
\hline Low speed & $\begin{array}{l}\text { Liftoff at low speeds is still empirical. } \\
\text { Usefulness is limited at low surface speeds. } \\
\text { Starting torques are high. }\end{array}$ \\
\hline Size & Bulky and limited load for cryogenic machines. \\
\hline
\end{tabular}

TABLE IV

Some Applications of Foil Bearings

\begin{tabular}{lcc}
\hline \multicolumn{1}{c}{ Application } & \multicolumn{1}{c}{ Type } & Manufacturer \\
\hline & Auxiliary air handlers & \\
Boeing 747 & Commercial & MTI \\
Mirage 2000 & Military & MTI \\
Falcon 900 & Business & MTI \\
Boeing 757, 767 & Commercial & Allied Signal \\
McDonnell Douglas DC-10 & Commerical & Allied Signal \\
& Testing & \\
Cryogenic turbomachine: & NASA & Allied Signal \\
$\quad$ Liquid hydrogen & & \\
Liquid nitrogen & & \\
Liquid oxygen & McDonnell Douglas-Northrup & Allied Signal \\
F16 and F18 & Army & Allied Signal \\
Abrams tank M1 & &
\end{tabular}




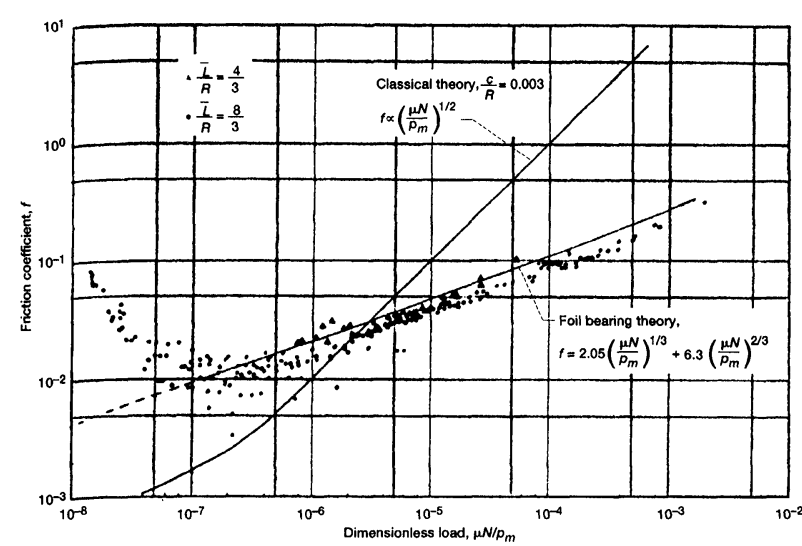

FIGURE 11 Coefficient of friction as a function of dimensionless loading parameter $\mu \mathrm{N} / p_{m}$. From Blok and van Rossum (1953).

[1990] and Ping and Carpino [1993] modeled the leaffoil concept and also produced loading and dynamic results (Fig. 13).

Foil-fluid-film behavior. The definition of the foil and its interaction with the adjacent foils relative to the "bumpy" support foils establish the load capacity. Fluid injected onto the foils is engaged by the loaded rotor to initiate a film wedge that deforms the foil. For the floating-ring foil configuration of Braun et al. [1994] the local flow field forms a pocket (see hydrostatic bearing) with a small vortex flow field mimicking third-body effects to form a pinch point (like the hydrostatic bearing) that supports the load. The cyclic behavior of the flow field over the entire bearing gives rise to an

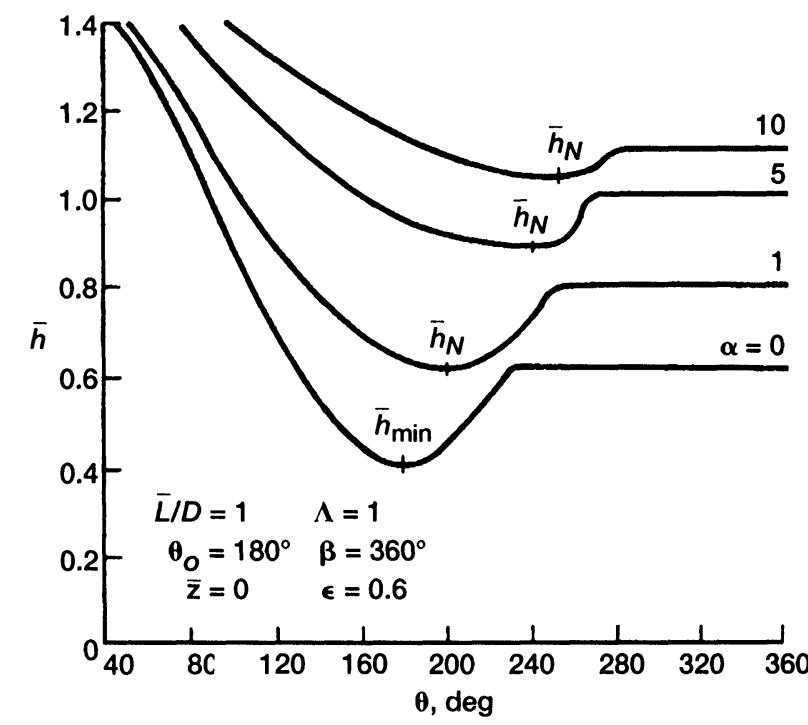

FIGURE 12 Nominal film thickness for bump-foil concept. From Heshmat et al. [1983]. instability, such as whirl. If the approach region is starved of injected fluid, intermittent foil contact can occur with significant instabilities and heating that destroy the bearing (and most likely the turbomachine).

The local foil mechanics and fluid distribution are very sensitive to the main-stream flow, the injected fluid, and boundary deformations. A second example of strongly coupled main-stream and secondary flow systems with the addition of compliant interfaces.
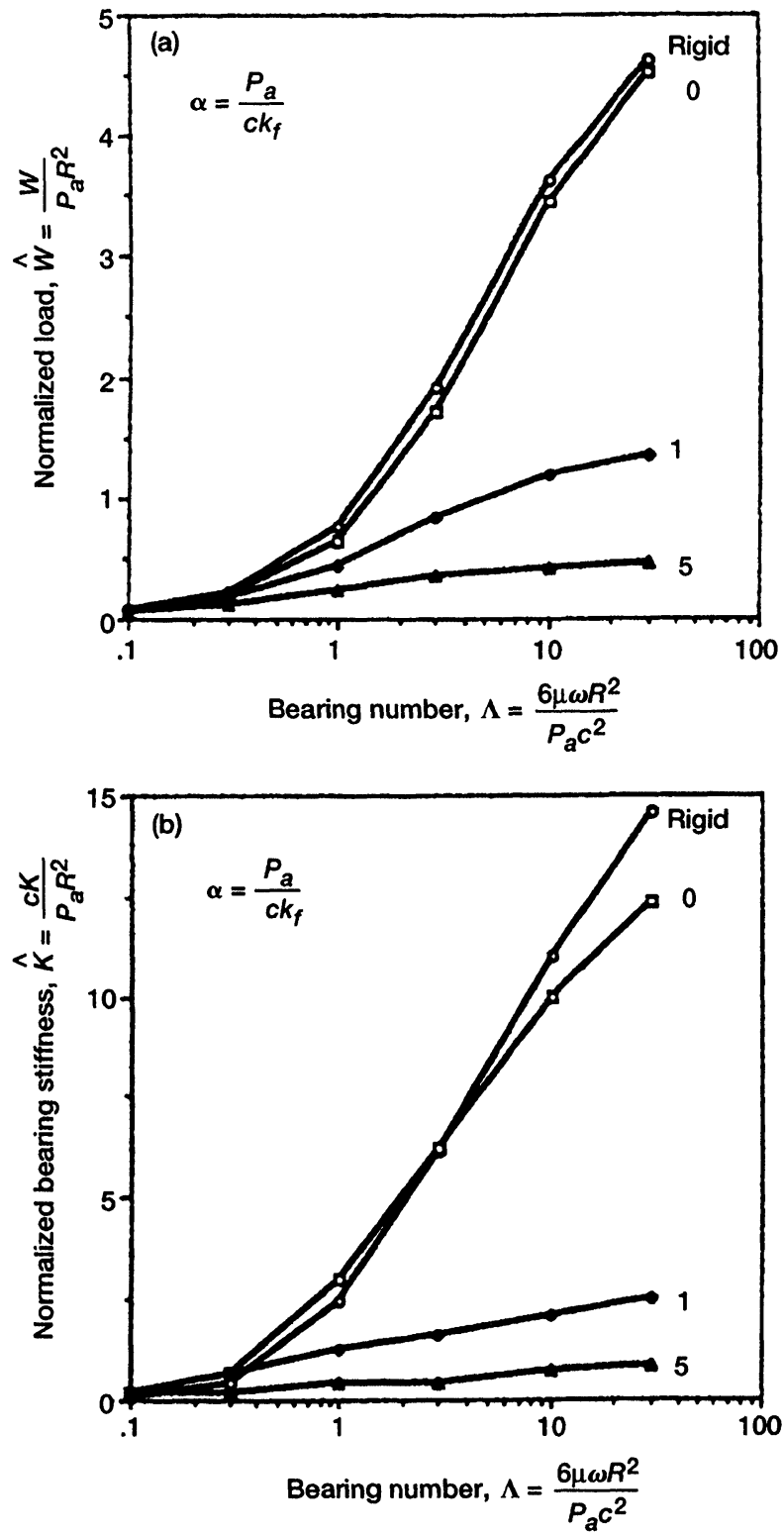

FIGURE 13 Foil-bearing parameters for leaf-foil concept as a function of bearing number with compliance as a parameter for length-todiameter ratio $\bar{L} / \mathrm{D}$ of 1 and eccentricity $\epsilon$ of 0.5 . From Ping and Carpino [1993]. (a) Normalized load. (b) Normalized stiffness. 


\section{BRUSH SEALING AND ENGINE TEST RESULTS}

The brush seal represents a compliant interface between a secondary flow system and the main-stream or powerstream flow. Brush seals can be effective seals, but their life and durability are not fully understood. Brush seal flow fields are complex in that a variety of flow phenomena occur within the brush. Figures 14(a) and 15(a) illustrate a two-dimensional computational fluid dynamics (CFD) computation of the flow field within a brush; associated experimental patterns are illustrated in Figures 14(b) and 15(b) and (c). In applications the bristles are in contact with a high-speed dynamic interface (unless the seal is a static type of brush). The bristle pressure at the interface is critical to preventing wear and excessive heat generation if the preload is too large and cooling flows are too low to remove the heat. Destruction of the seal (and sometimes the shaft) can result. However, all brush seal designs that were rig and engine tested at NASA Lewis have been successful in that they did not fail (Hendricks et al. [1993a,b; 1994]).

Both static and dynamic brush seal systems have been analyzed and tested for turbomachine applications (Ferguson [1988]; Chupp and Nelson [1990]; Chupp and Dowler [1991]; Holle and Krishnan [1990]; Flower [1990]; Carlile et al. [1992]; Hendricks et al. [1992]; Conner and Childs [1992], and Proctor et al. [1994]). Design issues are addressed by Basu et al. [1993]; Braun et al. [1990]; Braun and Kudriavtsev [1993]; Hendricks et al. [1991].

\section{Performance}

Brush seal integrity testing. In a low-speed, hard-rub bench test the brush seal bristles withstood over $1 \times 10^{9}$ cycles without pullout, fracture, or massive debris generation (Hendricks et al. [1993a]). A split-ring brush seal was then fabricated and tested between two honeycomblabyrinth shroud seals (Figs. 16 and 17) in the fourthstage turbine of a T-700 experimental engine (Hendricks et al. [1993b]). The brush seal withstood highly irregular surface operations at speeds to $335 \mathrm{~m} / \mathrm{s}(1100 \mathrm{ft} / \mathrm{s})$ and shroud temperatures to $620^{\circ} \mathrm{C}\left(1150^{\circ} \mathrm{F}\right)$. The turbine orbit was noncentered during steady and cyclic loading, and bristle wear was line to line.

Dual-brush compressor discharge pressure (CDP) test. In a later T-700 experimental engine test (Fig. 18) of the compressor discharge seal a CDP dual-brush seal outperformed the six-tooth-labyrinth seal (Hendricks et al. [1994]). The seal had a nominal diameter of $71 \mathrm{~mm}$ ( $2.8 \mathrm{in}$.) and was tested for $46 \mathrm{hr}$ of engine operations at pressures to $1 \mathrm{MPa}$ (145 psi), temperatures to $407^{\circ} \mathrm{C}$ $\left(765^{\circ} \mathrm{F}\right)$, and operating speeds to $160 \mathrm{~m} / \mathrm{s}(530 \mathrm{ft} / \mathrm{s})$. To within the uncertainty of the data and short engine hours (46 hr), experimental engine specific fuel consumption for the brush seal was reduced over 1 percent relative to the labyrinth seal performance (Fig. 19).

\section{Durability}

Tribological pairing. It is important that the seal perform as predicted and tribological pairing of the interface materials must be considered. Derby and England [1992] reported minimal brush and coating wear
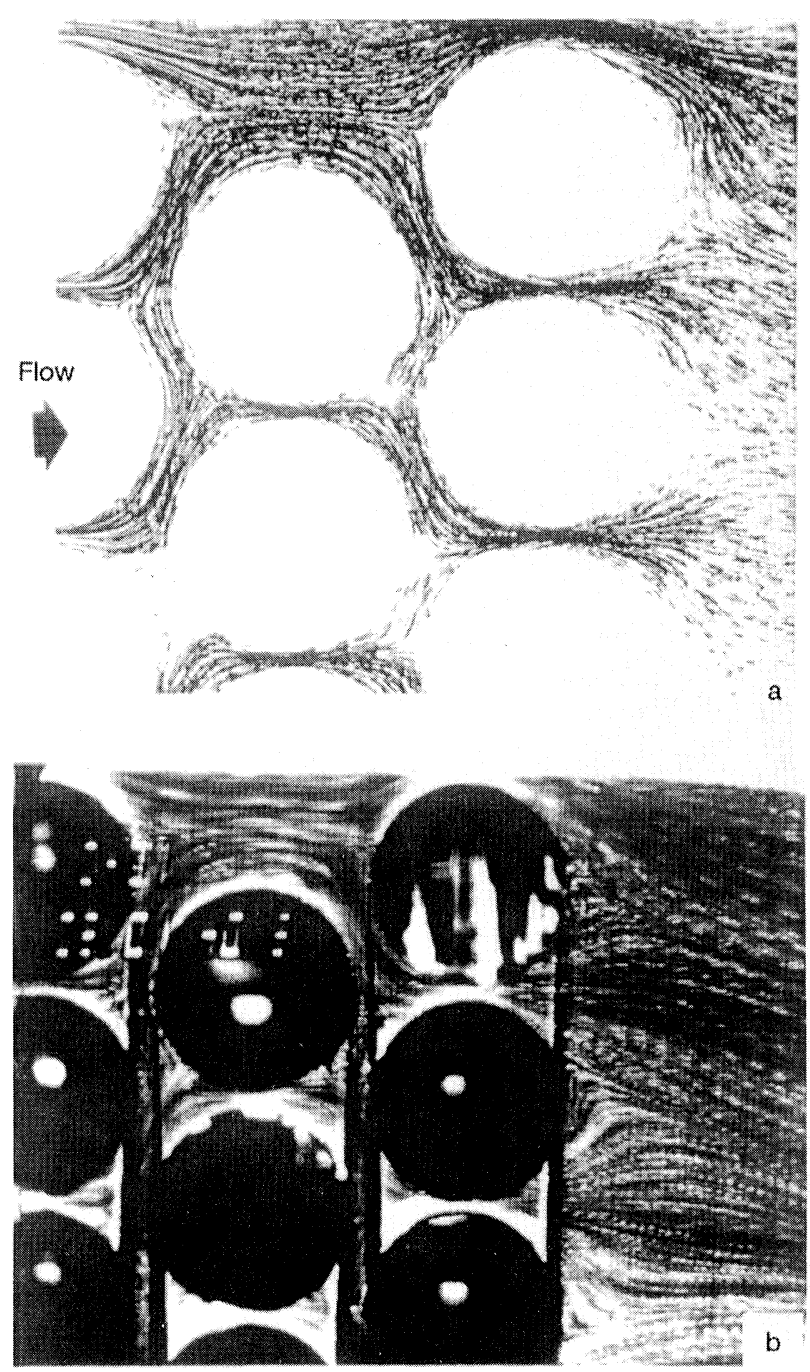

FIGURE 14 Two-dimensional laminar flows through staggered cylinders, simulating brush seal flows. (a) Calculated streamlines. (b) Experimental streak lines. 

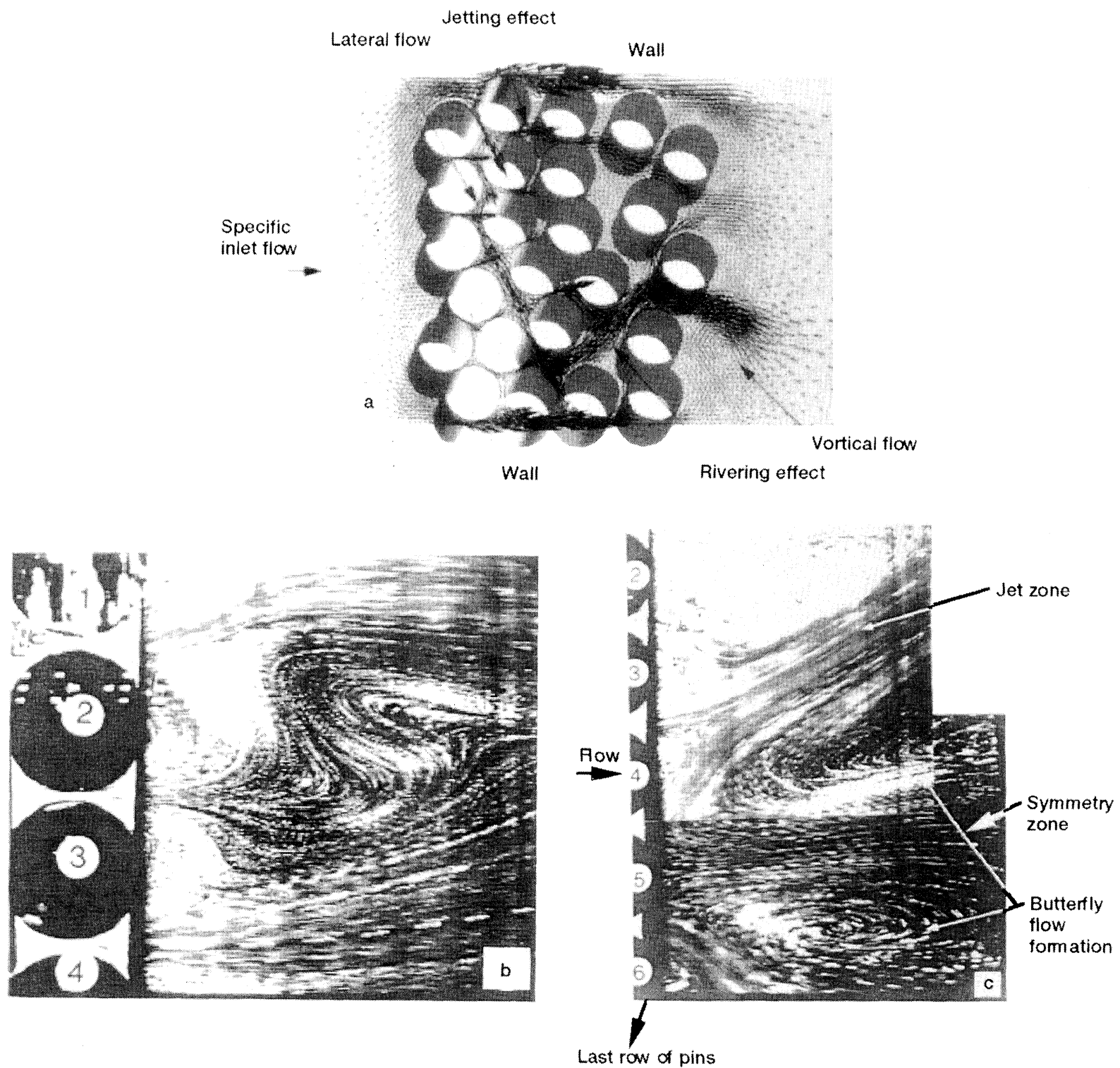

FIGURE 15 Flow field simulation at center plane of an array of cylinders, illustrating rivering, jetting, and vortical flow and experimental wake effects for uniform arrays. (a) Vector plot nonuniform array. (b) Wake flow near wall staggered array. (c) Wake flow near center staggered array.

using the Triboglide (modified chromium carbide $(\mathrm{CrC})$ ) coating and a nickel-based superalloy bristle in tests performed with air to $650^{\circ} \mathrm{C}\left(1200^{\circ} \mathrm{F}\right)$. Atkinson and Bristol [1992] reported less wear for a cobalt-based alloy rubbing against $\mathrm{CrC}$ at room temperature than for a nickel-based alloy and less leakage under dynamic conditions in a simulated CT7-9 compressor discharge seal test.

Bristle characteristics. In a T-700 experimental engine test (Hendricks et al. [1993b]) one metallo-graphic sample showed material smearing and a potential tungsten redistribution (Fig. 20(a)) that could indicate bristle melting at the interface. Shear smearing (forming mudflat cracks) was prevalent (Fig. 20(b)) as was oxide scale formation on the bristle (Fig. 20(c)) with various color gradations noted.

Bristle and interface characteristics. The dual-brush compressor discharge seal configuration and engine test- 


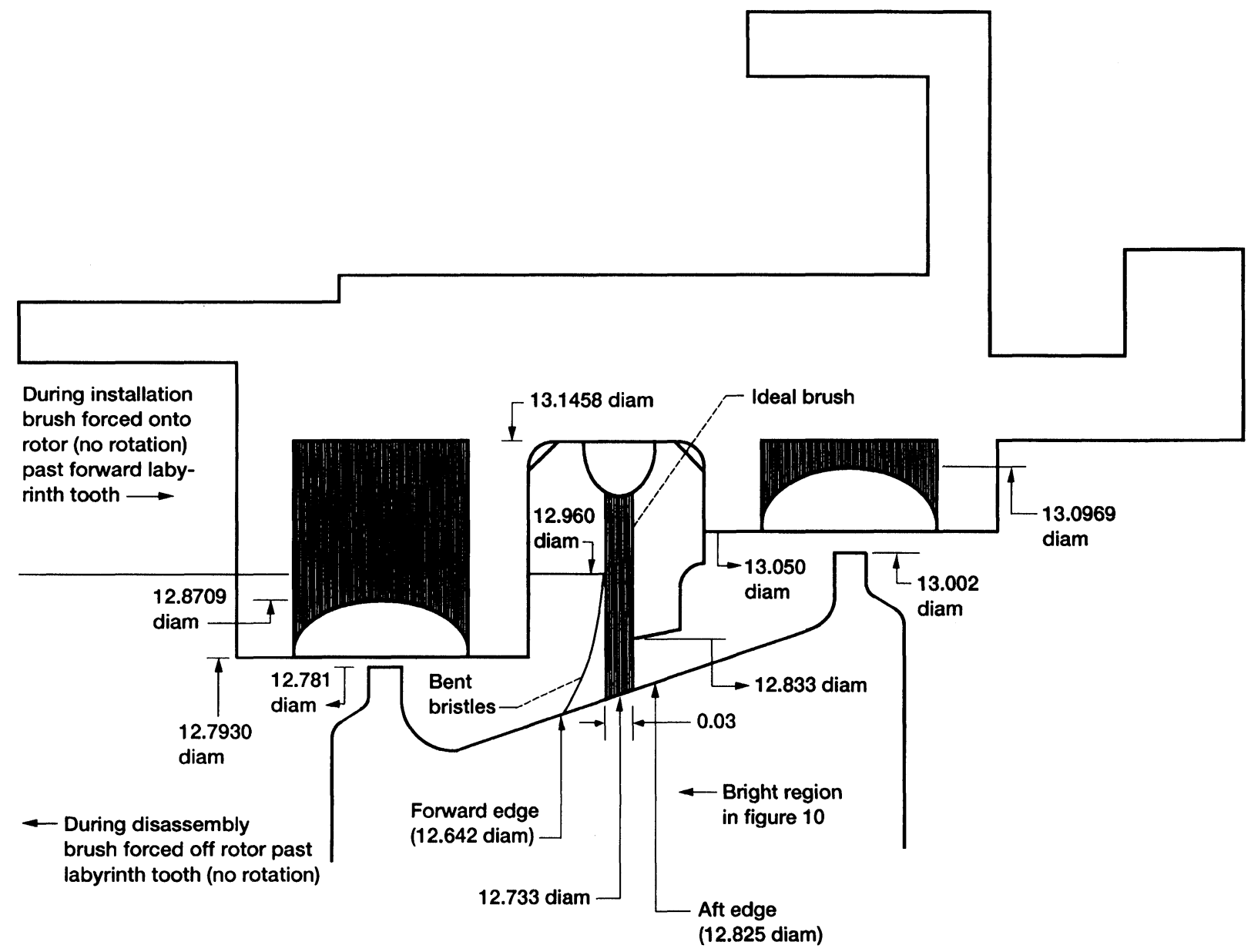

FIGURE 16 Cross-sectional view of split-ring brush seal installation (T-700 test engine).

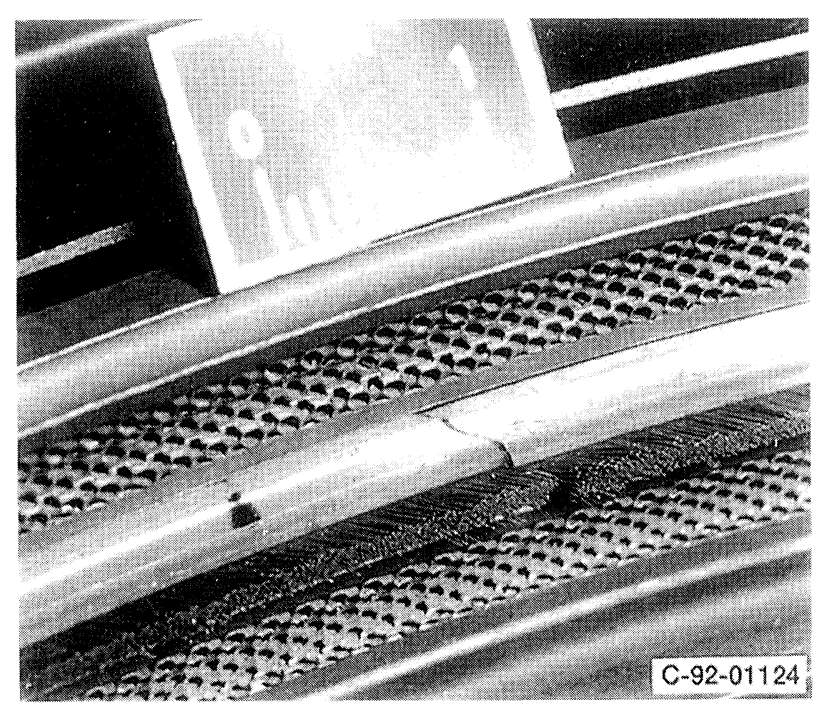

FIGURE 17 View of installed brush seal. ing (Hendricks et al. [1994]) showed more wear for the downstream brush (higher pressure drop enhancing bristle stiffness, Fig. 21(a)). The illustrated wear was associated with an assumed hard start or dynamic excursion; nominal wear was less than $5 \mu \mathrm{m}$ (0.002 in.). Metallographic analysis of the brush bristle wear bands and the matrix material (Figs. 21(b), (c), and (d)) showed little or no evidence of material loss or transfer. The bristle tips exhibited some shear smearing and oxidation (Fig. 22).

\section{Summary and Future Needs}

Interface tribology. The performance of the brush seal and its potential long-term advantages over the labyrinth seal have been demonstrated, but the tribological behavior of the interface is not understood.

Life prediction. It is important to develop simple prediction tools: (1) that couple the flow and heat transfer within the bristle pack and at the rotor-bristle interface 


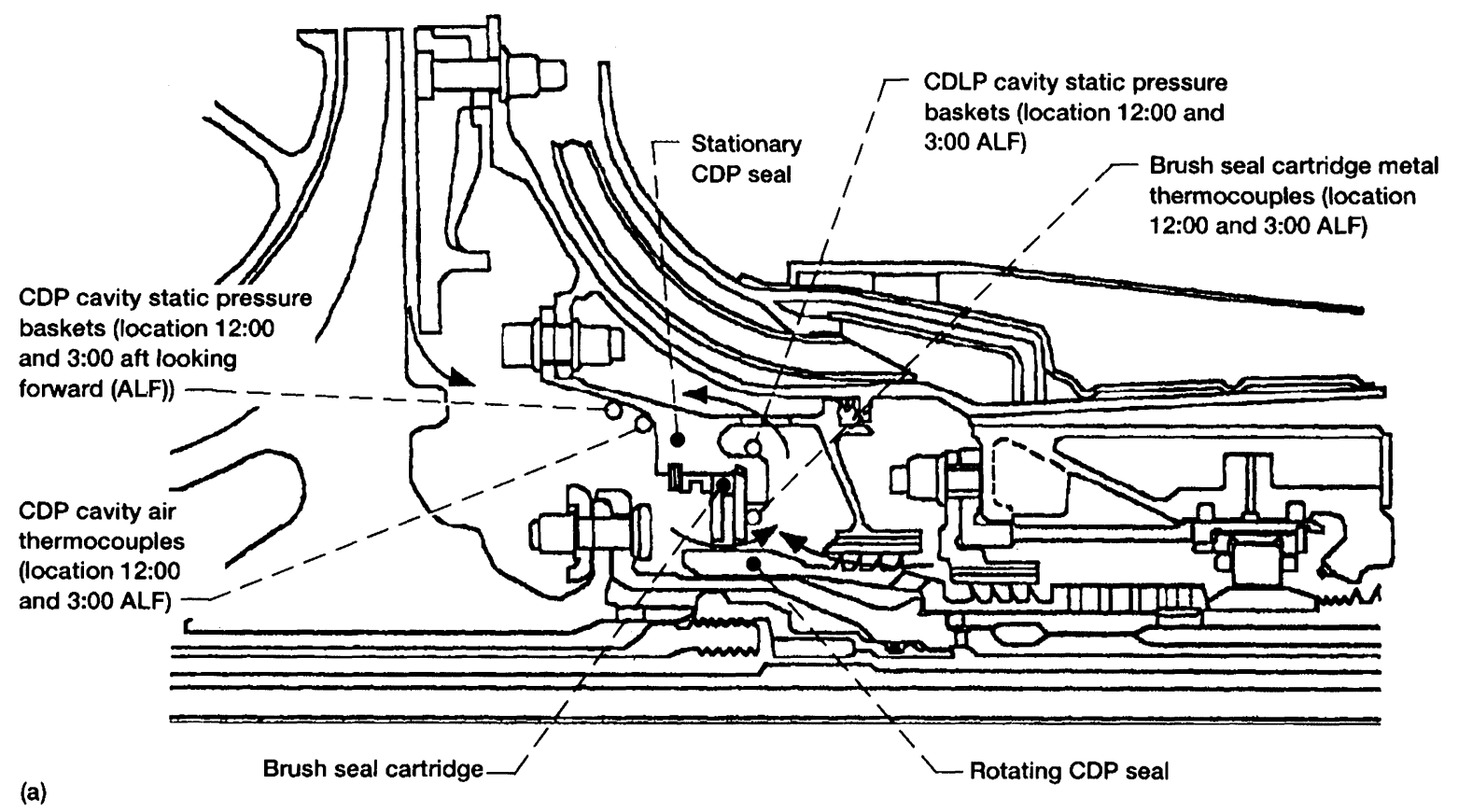

(a)

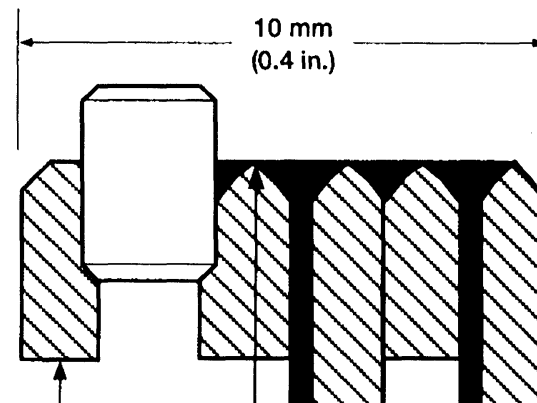
88.2-mm (3.47-in.) diam
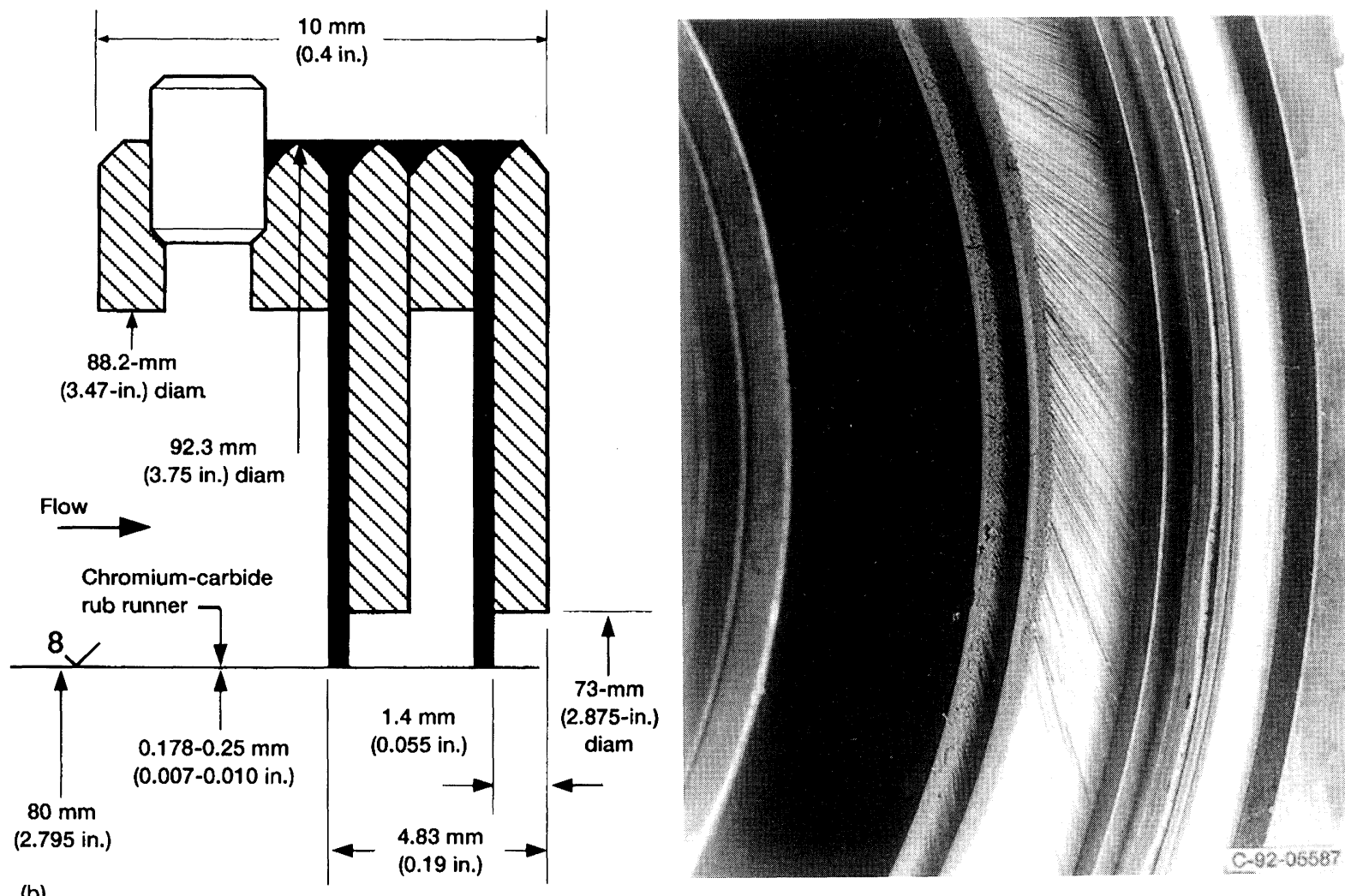

(b)

FIGURE 18 Dual-brush compressor discharge seal system and schematic of airflow. (a) Brush seal package and airflow. (b) Illustration of system. 


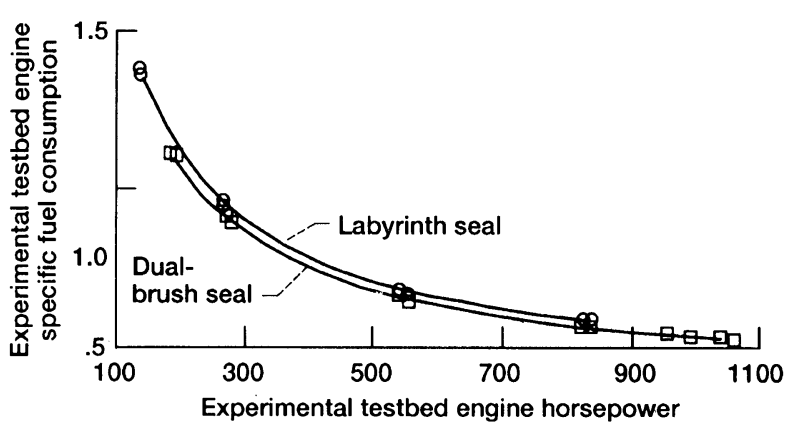

FIGURE 19 Experimental testbed engine specific fuel consumption as a function of horsepower.

including rotor and bristle dynamics and (2) that have a rub model for wear including tribological pairing of the interface materials.

Developments. It is necessary to develop (1) coatings and bristle materials that will enable brush seals to operate to $815^{\circ} \mathrm{C}\left(1500^{\circ} \mathrm{F}\right)$ ambient fluid temperature at surface speeds to $490 \mathrm{~m} / \mathrm{s}(1600 \mathrm{ft} / \mathrm{s})$ and (2) predictive capabilities that provide thermal management between the power stream and the secondary air systems associated with seal leakages that control cooling, lubrication, and turbomachine dynamics.

Engine test results present a third strongly coupled main-stream flow and compliant surface with a variety of secondary flow fields within the brush as well as interactions albeit unknown. For example, the compressor flow field in the CDP test (see sect. Dual Brush..CDP Test) enhanced compressor discharge pressure and resulting engine efficiency.
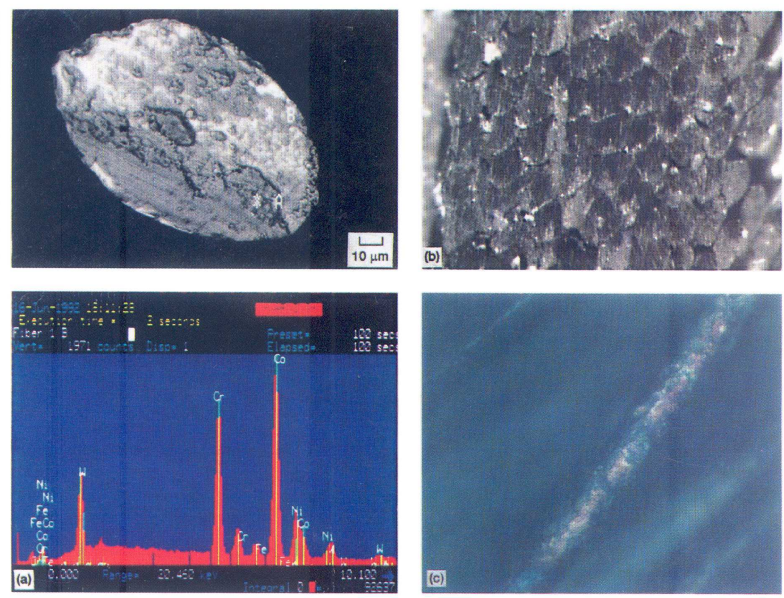

FIGURE 20 Post-test bristle metallographic results (T-700 fourthstage turbine test). (a) Bristle tip material smear. (b) Smearing at bristle interface. (c) Oxide scale of bristles.
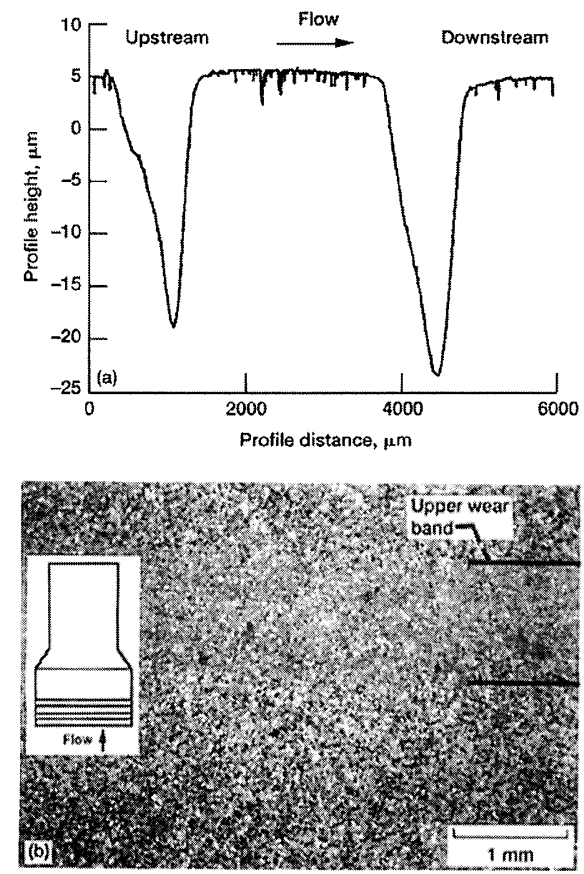

FIGURE 21 Post-test metallographic results of chromium-carbidecoated T-700 compressor discharge seal test rub-runner rotor for dual-brush seal. (a) Dual-brush wear scars over $120^{\circ}$ of rub-runner rotor. (b) Downstream wear band.
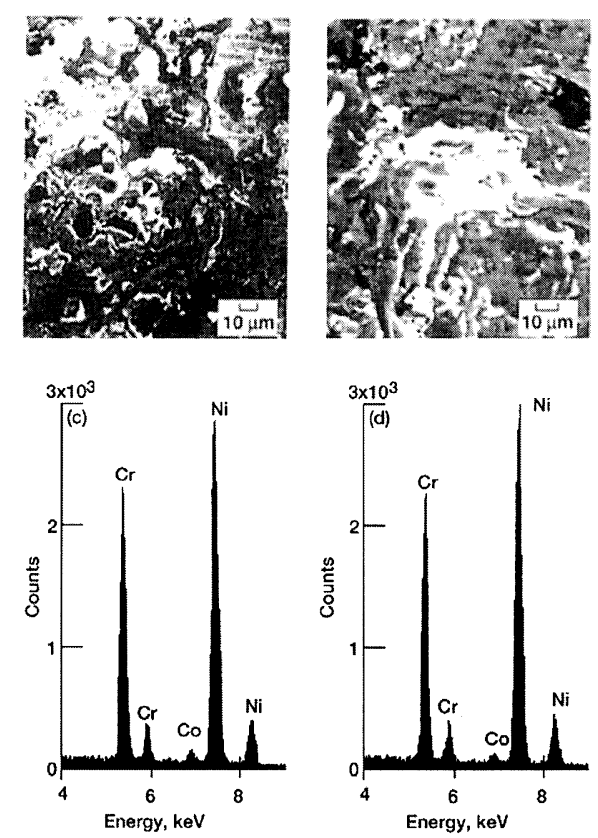

FIGURE 21 Continued. (c) SEM peaks for light area of downstream wear band. (d) SEM peaks for light area of matrix between wear bands. 


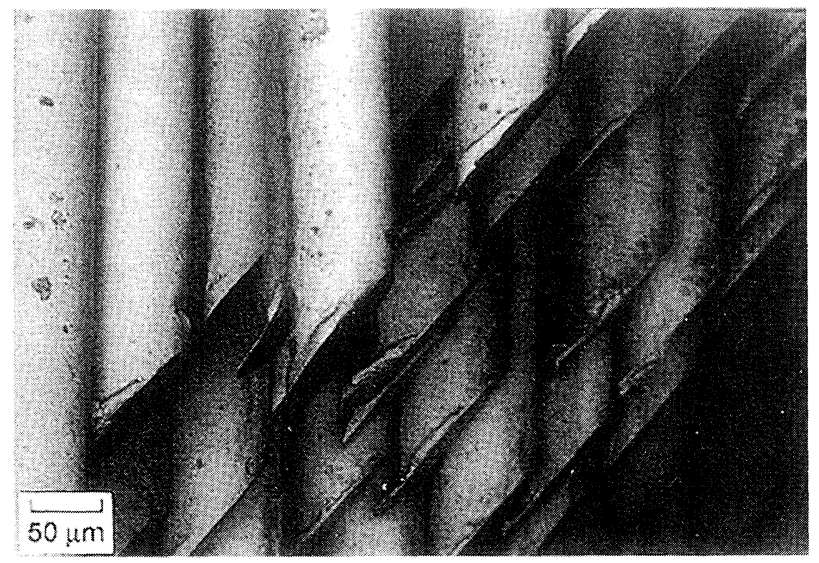

FIGURE 22 Closeup of bristles (compressor discharge seal test).

\section{SEAL-CAVITY-POWER-STREAM FLOWS}

Seals, bearings, cavities, and coolant and purge streams are secondary flow systems with common problems that are applicable across the board to all rotating powergenerating machines and compressors. There is strong interest in coupling the thermal management of the power stream, the secondary flow streams, and the seals within airbreathing and rocket turbomachines. Coupling would enable important interactions to be studied with limited resources before design commitment. Coupled analyses will include the flows above and below the blade platforms as well as to the bypass ducts with proper attention given to their interactive dynamics (turbomachine stability) (Fig. 23). Flows above the platform (power stream) usually have high pressure, high temperature, and high transonic Mach numbers; secondary flow streams have low Mach numbers and cool fluids.

\section{SCISEAL-ADPAC COUPLING}

Two CFD codes developed under different NASA contracts target the two principal gas paths in the turbomachine. A brief description of each of the codes, their merits, and their important relevant features is given here.

ADPAC. The ADPAC code was modified from an earlier NASA code (Adamczyk et al. [1990]) by Allison Gas Turbine Engine Division under a NASA Lewis contract (Hall and Delaney [1993a,b]) for the treatment of transonic flows in turbines, compressors, and fans. The code uses a strongly conservative form of the compressible Navier-Stokes flow equations. An explicit, multistage Runge-Kutta procedure is used to integrate

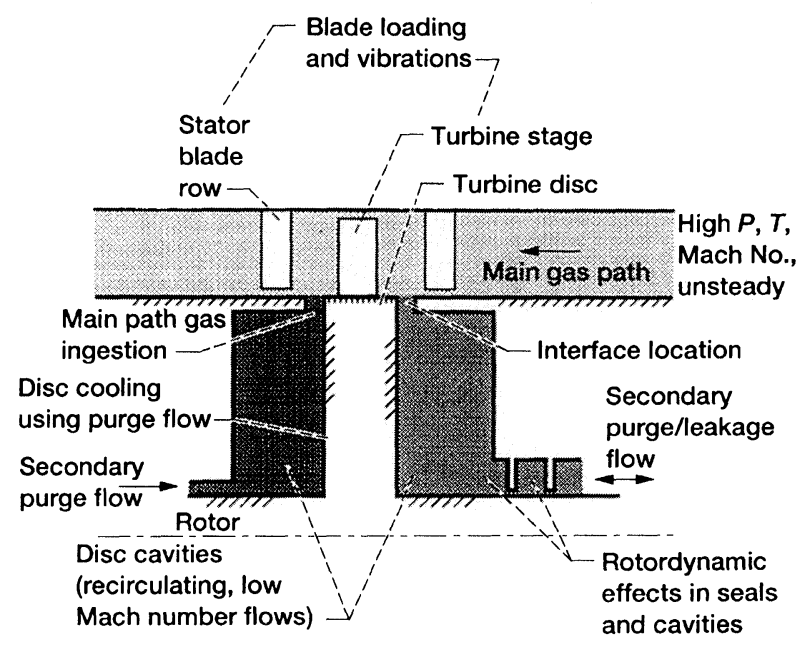

FIGURE 23 Schematic of typical turbine stage and associated disc cavities and seals.

the density-based equations in time so as to get timeaccurate as well as steady-state solutions. The code can treat multidomain (block) grid. Convergence acceleration methods, such as residual smoothing and multigrid solutions, are available for steady-state solutions. ADPAC can treat the time-dependent flow that exists in multiple rows of stationary and rotating blades by using a circumferential averaging procedure to transfer data across the boundaries of the moving and stationary blade rows. The Baldwin-Lomax model is used for turbulent flows.

SCISEAL. The SCISEAL code was also developed under a NASA Lewis contract by CFD Research Corporation (Athavale et al. [1994]) for the treatment of various types of turbomachinery seals. It uses a finitevolume, pressure-based method to integrate turbulent Navier-Stokes flow equations. The solution methodology allows solutions of very low to high Mach number compressible and incompressible flows. Several types of turbulence models are available, including standard, low Reynolds number, multiple-scale, and two-layer models. Both stationary and rotating coordinates can be used, and the code can treat the high-aspect-ratio geometries encountered in secondary flows. Two automated computational procedures are available in the code for computing rotordynamic coefficients: (1) circular whirl orbit method for centered rotors and (2) a small perturbation-based method for eccentric and/or misaligned rotors.

Coupling methodology. The flow in the main gas path in the turbomachine section is usually in the transonic range, and hence the density-based formulation has been 
employed in ADPAC. The SCISEAL code uses a pressure-based formulation to deal with the near-incompressible and very low Mach number flows that often exist in the secondary path. A flow chart of the proposed coupled execution procedure is illustrated in Figure 24.

The following problems have been encountered and resolved: (1) left (ADPAC)- and right-hand coordinate system transformations are required; (2) solution methods are pressure driven and density driven (ADPAC); and (3) boundary gradients between the codes have to be established by using phantom points. Potential problems of rotional speed limitation (ADPAC) cited by Allison have not yet been encountered.

ADPAC is known to the industrial community and original equipment manufacturers. Although difficult to adapt, it will provide an initial acceptable solution to the problem. Other power-stream turbomachinery codes are available and have limitations but should be investigated.

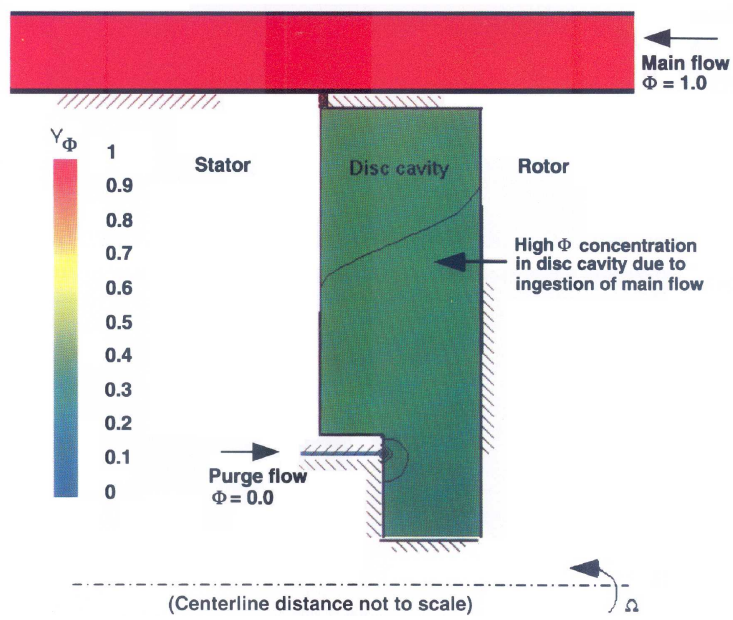

FIGURE 25 Calculated cooling effectiveness parameter $\varnothing$ in a generic disc cavity and rim seal.

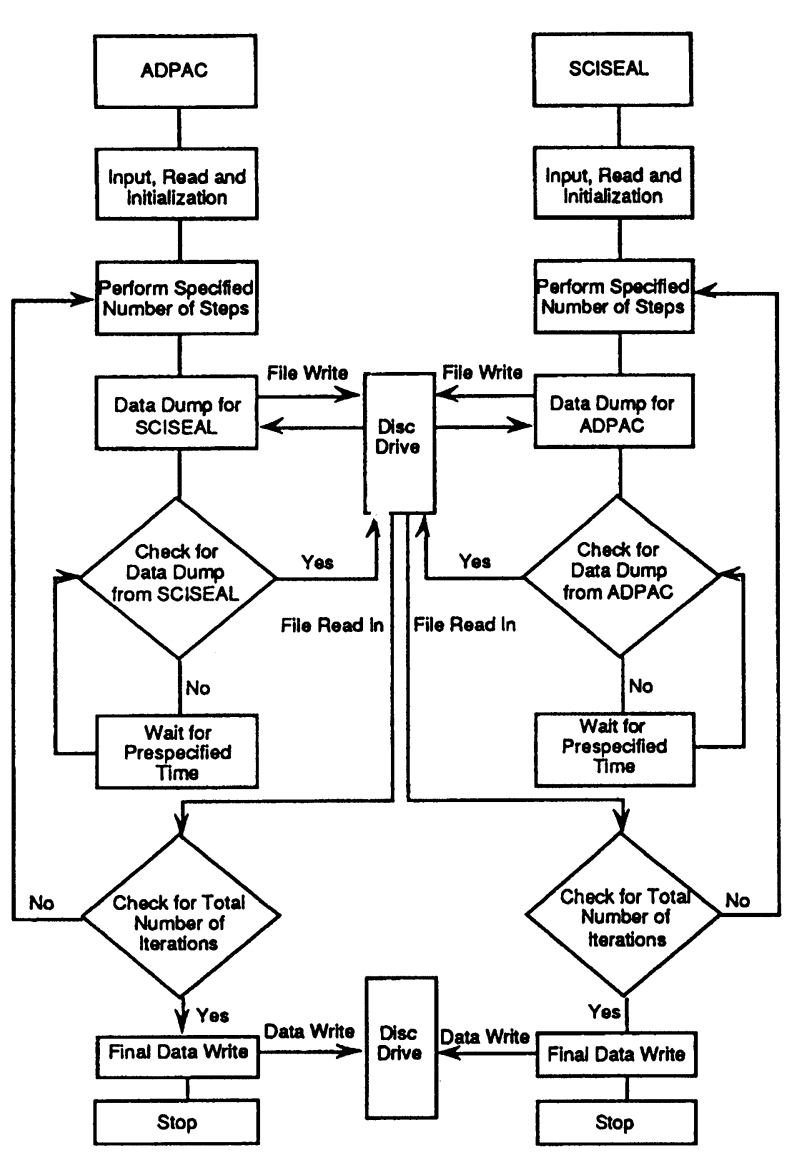

FIGURE 24 Coupled execution of ADPAC and SCISEAL.

\section{Rim Seal and Cavity Purge Results}

Code validation check. The example problems being used to benchmark the coupled codes are summarized here to provide some understanding of code capabilities and can be expanded in more detail in a series of presentations. The generic disc-cavity-power-stream interaction problem of gas mixing with high concentrations indicates gas ingestion (Fig. 25).

Daily and Nece [1960] enclosed-cavity flows and Prof. Owen's (Bayley and Owen [1970], Phadke and Owen [1983], and Gan et al. [1993]) cavity flow and rim seal measurements provide basic checks for codes. The results of Daily and Nece [1960] have been used in prior validation of the SCISEAL code but have not been used within the coupled codes. Copies of validation checks are included in Athavale et al. [1992].

SCISEAL and UTRC cavity results. Bruce Johnson's United Technologies Research Center (UTRC) cavity flow and rim seal data are reported by Graber et al. [1987]; the generic cavity and parametric definitions are given in Figure 26. Four cavity configurations (Fig. 27) were gridded, flow vector plots were produced, and carbon dioxide $\left(\mathrm{CO}_{2}\right)$ mixture concentration contours were mapped. These calculated results provide insights into the flow field and concentration patterns engendered by geometric changes and variations in the cooling effectiveness parameter $\phi$ or the ratio of the $\mathrm{CO}_{2}$ mixture of purge gas flow to the difference between purge and ingested flow $\phi=\left(C-C_{o} /\left(C_{p}-C_{o}\right)=\dot{m}_{p} /\left(\dot{m}_{p}-\dot{m}_{i}\right)\right.$ as a function of the purge-to-tangential Reynolds number 


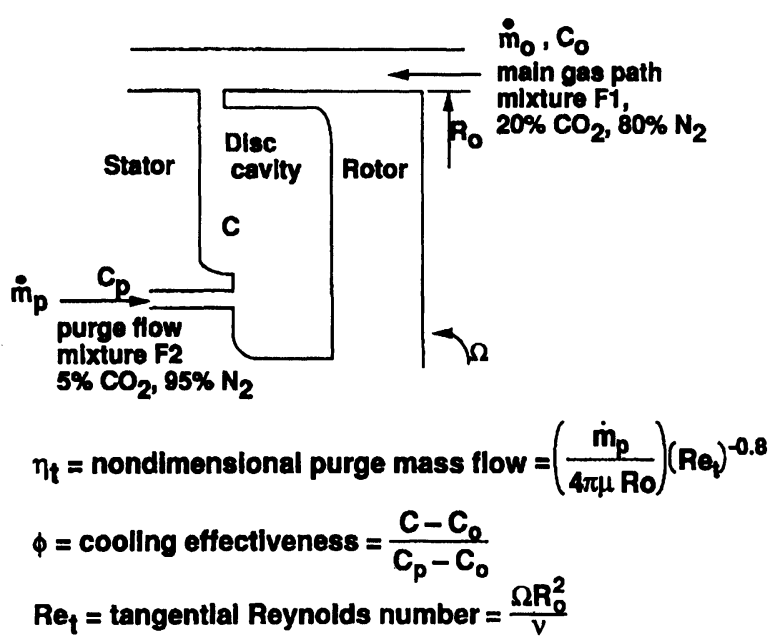

FIGURE 26 Generic disc cavity and rim seal notation with purge gas and main gas path mixture fractions for UTRC cavity-seal flows.

$\operatorname{Re}_{p} / \operatorname{Re}_{t}$ or in terms of the dimensionless coolant flow parameter $\eta_{t} \equiv\left(\dot{m}_{p} / 4 \pi \mu R\right) / \operatorname{Re}_{t}^{0.8}$.

The boundary conditions for the main passage flow as well as those for the purge flow need more careful
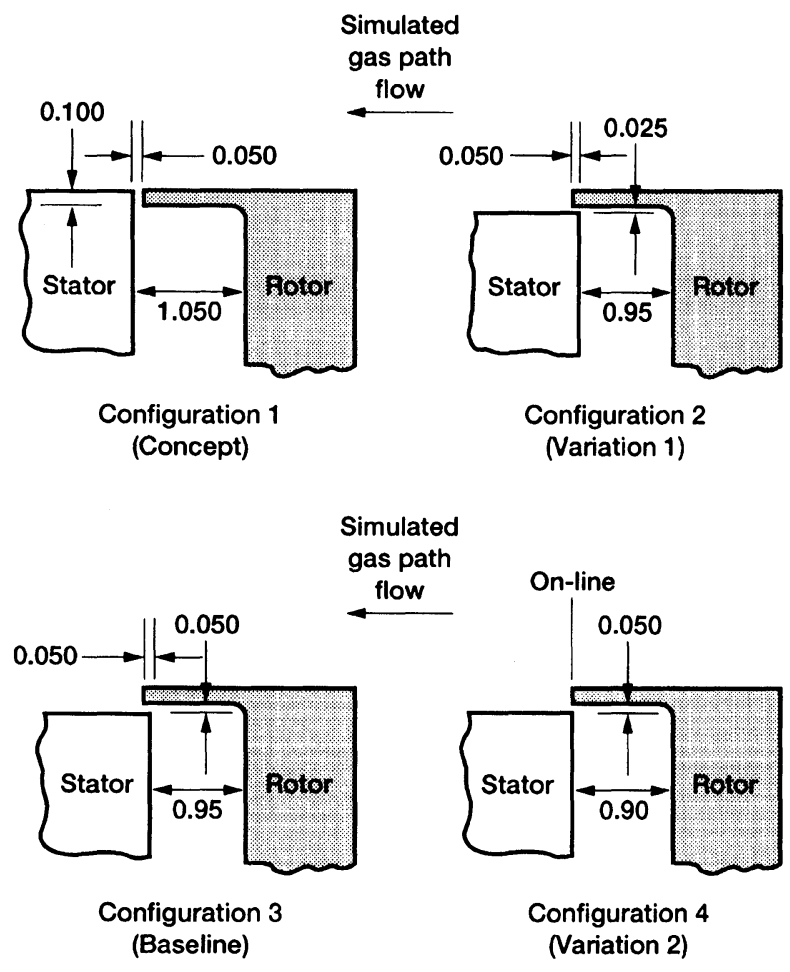

FIGURE 27 Four rim seal configurations (rotor diameter, $\sim 11.0$ in.). (Dimensions are in inches.) From Graber et al. [1987]. definition. A similar problem was encountered with the turbulent flow measurement data of Dr. Morrison at Texas A\&M (Morrison et al. [1994]) and required careful upstream pressure and shear measurements. The problem, which is still under study, is important to any future measurements of cavity flow.

The variation of $\phi$ due to purge mass flow at a fixed inlet swirl Reynolds number was calculated for four configurations measured by Graber et al. [1987] (Fig. 27). Results were calculated for each configuration at two purge flow rates $\eta_{t} \equiv 10^{-3}$ and $\eta_{t}=8 \times 10^{-3}$ (four purge flows for configuration 1). The ingestive experiments using different $\mathrm{CO}_{2}$ mass fractions in the flow streams were simulated by using passive scalar transport of inert species or mass diffusion. The calculations were carried out on grids of 50 to 60 cells in the axial direction and 60 to 70 cells in the radial direction. The rotor speed was constant at $2450 \mathrm{rpm}\left(V_{\theta}=69 \mathrm{~m} / \mathrm{s}(225 \mathrm{ft} / \mathrm{s})\right)$ with $\operatorname{Re}_{\mathrm{t}}=$ $5 \times 10^{6}$ and the inlet swirl $V_{\theta}=R \Omega=$ rotor tangential velocity. The results herein were calculated for two different sets of main passage flow velocities 2 to $20 \mathrm{~m} / \mathrm{s}$ ( 6.6 to $66 \mathrm{ft} / \mathrm{s})$ and 120 to $150 \mathrm{~m} / \mathrm{s}(390$ to $490 \mathrm{ft} / \mathrm{s})$.

High purge-flow-to-main-flow ratio: For main passage flow of 2 to $20 \mathrm{~m} / \mathrm{s}(6.6$ to $66 \mathrm{ft} / \mathrm{s})$ the cooling effectiveness was very good, and little or no ingestion was noted from the concentration contour maps (Fig. 28). Excessive parasitic coolant flows, although effective at cavity purging (Fig. 28(b)), increased the engine specific fuel consumption (SFC) or decreased the engine specific impulse $I_{\text {sp }}$.

Low-purge-flow-to-main-flow ratio: Increasing the main passage flow to 120 to $150 \mathrm{~m} / \mathrm{s}(390$ to $490 \mathrm{ft} / \mathrm{s})$ provided a more realistic comparison with data reported by Graber et al. [1987] and certainly showed gas ingestion at low $\eta_{t}$ (a function of $\operatorname{Re}_{p} / \operatorname{Re}_{t}$ ). The cavity flow and detail near the rim seal for configuration 1 at $\eta_{t}=$ $10^{-3}$ showed a large vortex within the cavity, several smaller vortices, and a significant vortex in the rim seal "neck" (Fig. 29(a)). The mixture fraction contours reflected ingestion (Fig. 29(b)). See Figure 26 for inlet mixture fractions of $5 \% \mathrm{CO}_{2}$ purge flow and $20 \% \mathrm{CO}_{2}$ main gas rate flow. Increasing $\eta_{t}$ to $8 \times 10^{-3}$ diminished both the cavity vortex and the rim seal "neck" vortex (Fig. 29(c)). The mixture plots (Fig. 29(d)) confirmed the effectiveness (or lack) of the purge.

Local velocity plots for configurations 1 to 4 (Fig. 30) with $\eta_{t}=10^{-3}$ and $\eta_{t}=8 \times 10^{-3}$ show that configurations 1,3 , and 4 had vortex flows within or near the rim seal "neck" at $\eta_{t}=10^{-3}$; all four configurations were effective at $\eta_{t}=8 \times 10^{-3}$. The experimental data and calculated results for $V_{\not \mathrm{j}} \approx R \Omega$ and $\mathrm{Re}_{t}=5 \times 10^{6}$ agreed well for all four cavity geometries over the $\eta_{t}$ range (Fig. 31 ). The 

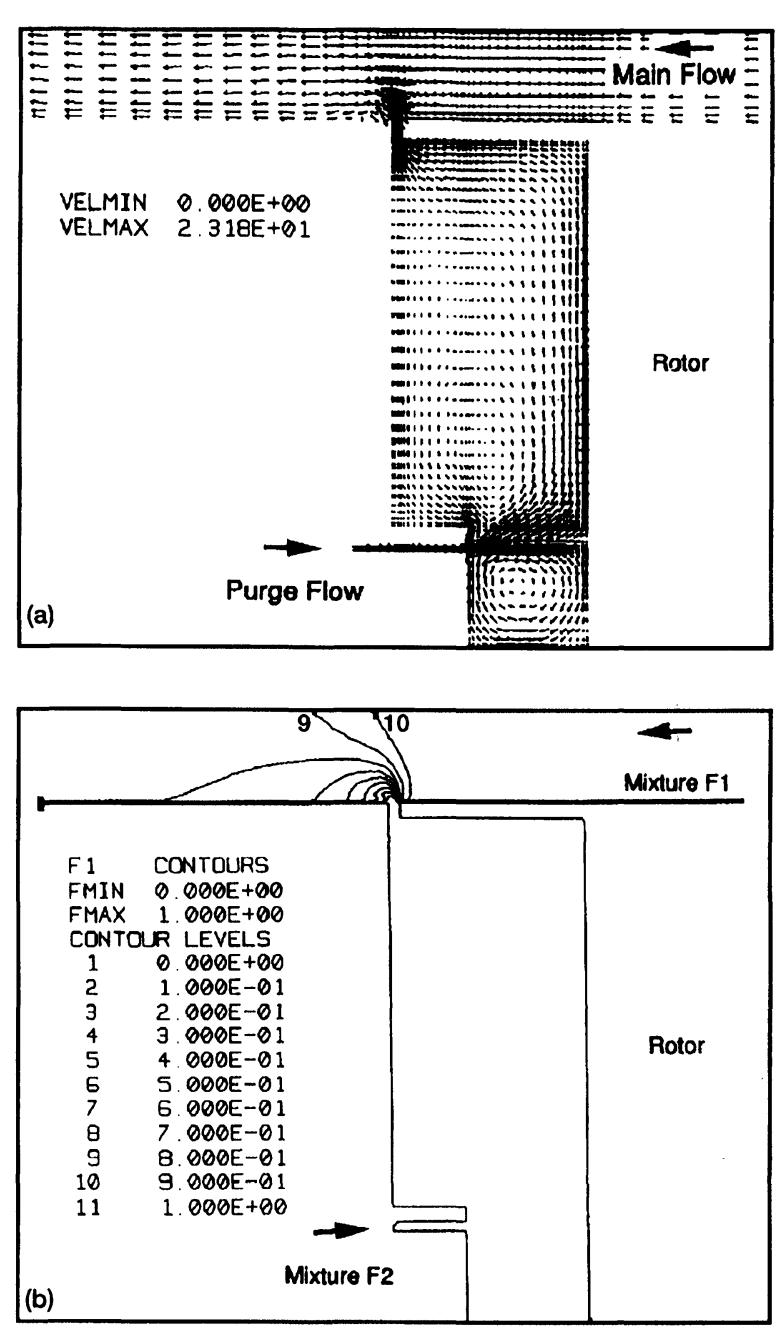

FIGURE 28 SCISEAL results for UTRC disc cavity configuration 1 with main gas path flow of 2 to $20 \mathrm{~m} / \mathrm{s}$ and purge parameter $\eta_{t}$ of $8 \times 10^{-3}$. (a) Velocity vector plot. (b) Mixture fraction contours.

calculated and experimental cooling effectiveness parameters $\phi$ were compared at $\eta_{t}=10^{-3}$ and $\eta_{t}=8 \times 10^{-3}$. Additional computations for $\eta_{t}=2 \times 10^{-3}$ and $\eta_{t}=4 \times 10^{-3}$ were completed for configuration 1 . The calculated to experimental differences, which ranged from 7 to 18 percent at $\eta_{t}=10^{-3}$ and \pm 2 percent at $\eta_{t}=8 \times 10^{-3}$, are summarized in table $\mathrm{V}$.

SSME turbomachine simulation. NASA Marshall Space Flight Center (Benjamin and McConnaughey [1991]) contracted UTRC to provide detailed data for cavity flows and interaction with the turbine disc to be used in aerodynamics and heat transfer studies of the SSME turbomachines. The data are specifically aimed at the high-pressure fuel turbopump (Daniels and Johnson [1993]).

Flow streams: The power-stream and coolingstream interactions indicate potential for hydrogen-rich steam to be ingested through the blade platform "fishmouth" seal on the high-pressure side of the turbine and then into the coupled cavities and through the platform interstage seals (Fig. 32).

In the UTRC tests the simulated cavities and purge flows were separated into six regions (Figs. 33 and 34). The associated $\mathrm{CO}_{2}$ gas source measurement results for region II, where $\phi=\left(C-C_{o}\right) /\left(C_{p}-C_{o}\right)$ (see Fig. 26), are shown in Table V.

Purge flow coupling: Purge flow calculations must account not only for power-stream interactions but for purge flows from other sources (i.e., a systems approach is required). Figure 35 illustrates the grid used and the purge flows calculated for region II without accounting for adjacent cavity purge carryover (Figs. 32 to 34 ).

\section{Associated Power-Stream and Secondary-Flow- Stream Results}

Lid-driven flow blade simulation. Athavale et al. [1993] investigated the three-dimensional flow in a lid-driven cavity with periodic boundary conditions, simulating flows in an unshrouded turbine blade passage (Fig. 36). The length of the tee-shaped cavity passage (Fig. 36(a)) was fixed at $\bar{L}_{\phi}=1$, the passage depth at $d / \bar{L}_{\phi}=0.5$, and the clearance at $c / \overline{\mathrm{L}}_{\phi}=0.025$; the maximum pressure $p_{\max }=1.01 \mathrm{MPa}(10 \mathrm{~atm})$ was set upstream at $\bar{L}_{\mu} / \bar{L}_{\phi}=0.2$ and the minimum pressure $p_{\min }=$ $0.81 \mathrm{MPa}(8 \mathrm{~atm})$ was set downstream at $\bar{L}_{d} / \bar{L}_{\phi}=0.2$ with the top wall moving at $150 \mathrm{~m} / \mathrm{s}(490 \mathrm{ft} / \mathrm{s})$. The code was benchmarked against the conventional lid-driven cavity calculations of Ghia et al. [1982] and the enclosed-rotor data of Daily and Nece [1960].

In general, the calculations show that flow due to the "blade" passage was carried over and initiated a vortex on the opposite wall (pressure side). The vortex pattern developed from this small region near the top of the "blade" leading edge and filled nearly one-third of the passage near the trailing edge. At higher rotational speeds the annulus undergoes Taylor instability, and a number of periodic asymmetric vortices appear, as initially found by Hayase et al. [1990].

The interesting feature is the introduction of counterand corotating control vortices at specific locations in the flow field. Figure 36(b) illustrates the base case with no controlled vortex injection. A corotating vortex near the pressure side (10 percent of the lid-driven vortex) can 


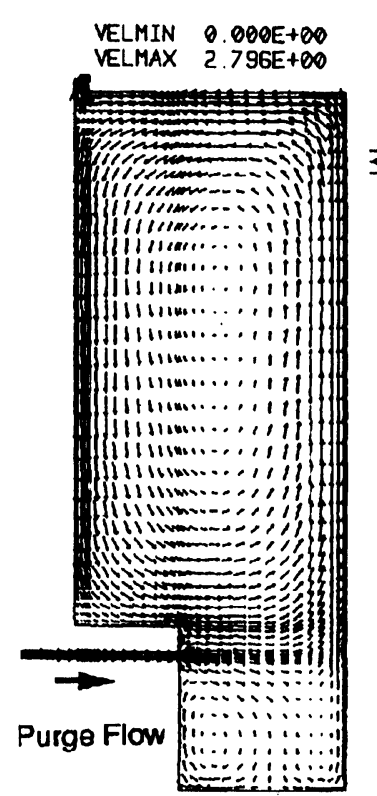

Flow Detail in the cavity

(a)

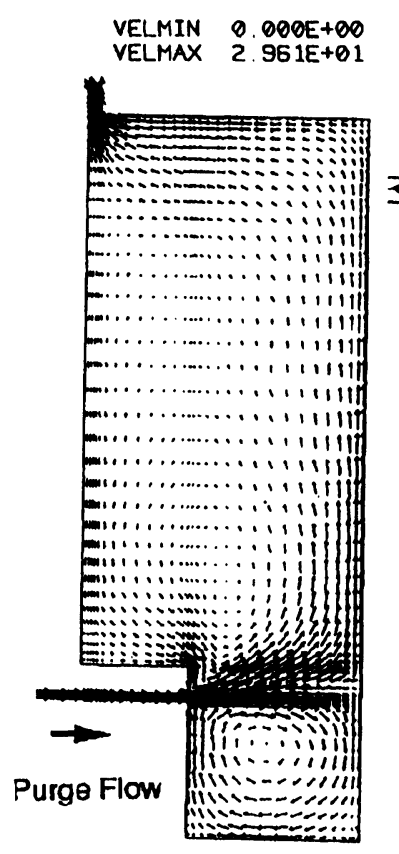

Flow Detail in the cavity

(c)

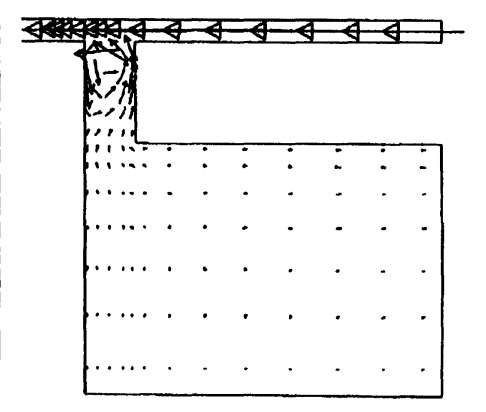

\section{VELMIN $9.000 E+\infty$
VELMNX}

Detail near rim seal

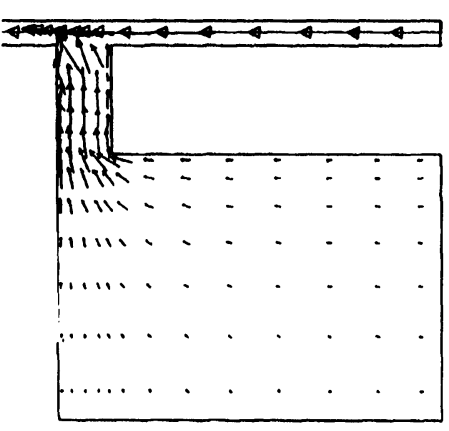

$\begin{array}{ll}\text { VELMIN } & 0.000 E+\infty 00 \\ \text { VELMAX } & 1.839 E+\varnothing 2\end{array}$

Detail near rim seal

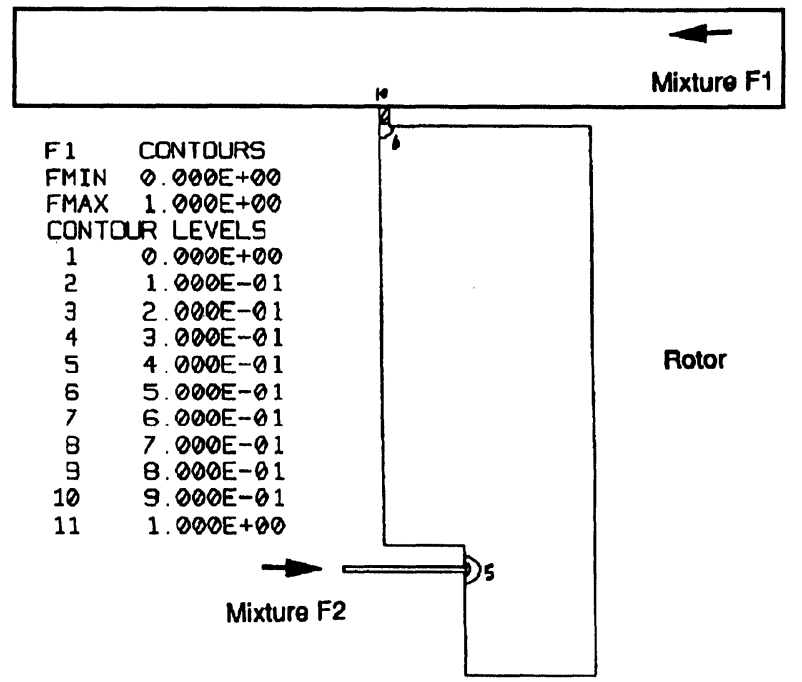

(b)

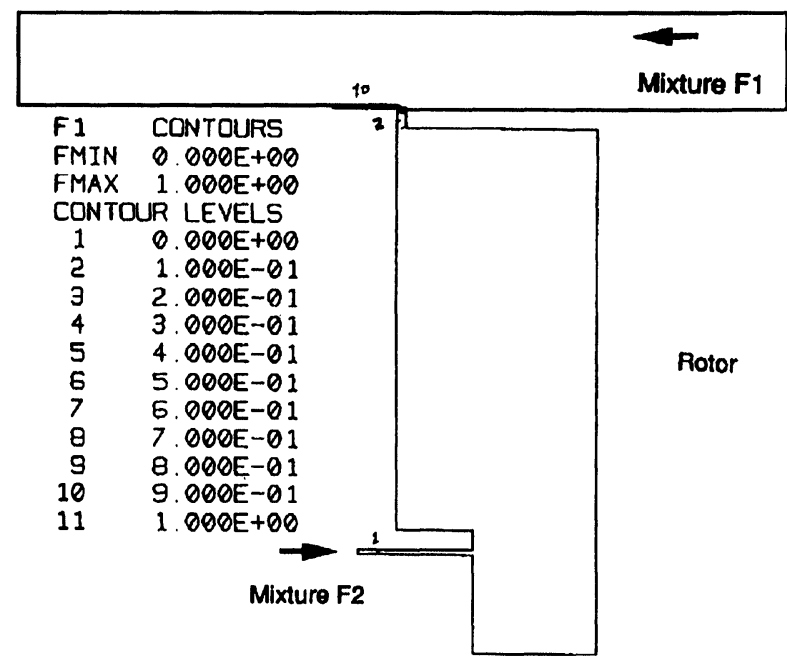

(d)

FIGURE 29 SCISEAL results for UTRC disc cavity configuration 1 with main gas path flow of 120 to $150 \mathrm{~m} / \mathrm{s}$ for two purge parameters $\eta_{t}=$ $10^{-3}$ and $\eta_{t}=8 \times 10^{-3}$. (a) Velocity vector plot $\left(\eta_{t}=10^{-3}\right)$. (b) Mixture fraction contours $\left(\eta_{t}=10^{-3}\right)$. (c) Velocity vector plot $\left(\eta_{t}=8 \times 10^{-3}\right)$. (d) Mixture fraction contours $\left(\eta_{t}=8 \times 10^{-3}\right)$. 


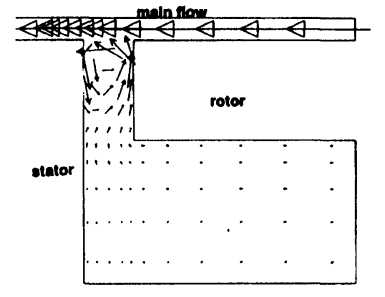

(a)

$\eta_{t}=1 \times 10^{-3}$

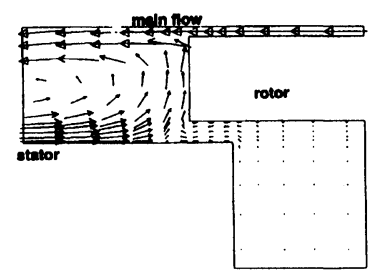

(b)

$$
\eta_{t}=1 \times 10^{-3}
$$

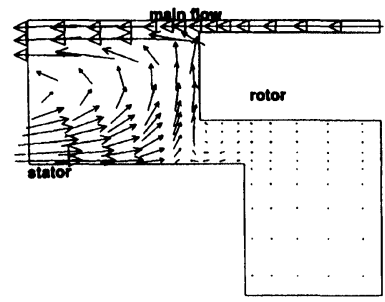

$n_{t}=1 \times 10^{-3}$

(c)

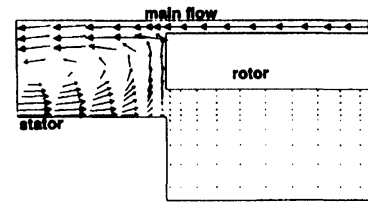

(d)

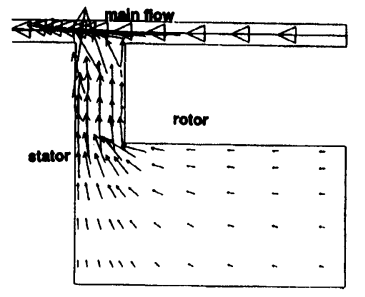

$\eta_{t}=8 \times 10^{-3}$

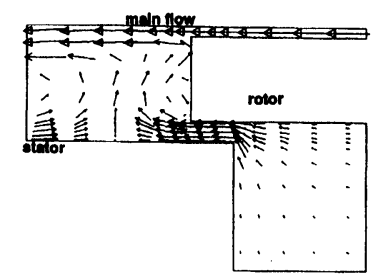

$\eta_{t}=8 \times 10^{-3}$

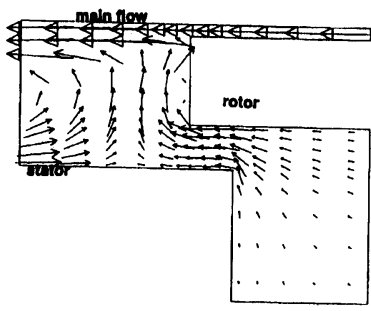

$\eta_{t}=8 \times 10^{-3}$

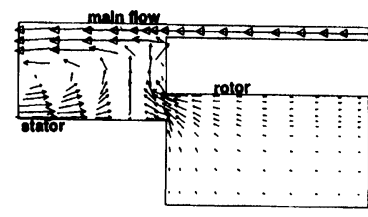

$\eta_{t}=8 \times 10^{-3}$
FIGURE 30 Comparison of flow details at rim seal power-stream interface for four UTRC cavity and seal configurations at two purge parameters $\left(\eta_{t}=10^{-3}\right.$ and $\eta_{t}=8 \times 10^{-3}$; see also figure 27). (a) Configuration 1. (b) Configuration 2. (c) Configuration 3. (d) Configuration 4 .

cause flow reversals; when the vortex was placed near the suction side, no flow reversal was noted (Figs. 36(c) and (d)). Introducing a counterrotating vortex near the suction surface reduced the lid-driven flow vortex and extended it to midcavity (axial) with a nearly centered vortex pattern near the axial exit plane (Figs. 36(d) and (e)). A counterrotating vortex introduced near the pressure side caused a strong opposition to the flow near the inlet and a double vortex pattern.

Disc cavity flow simulation. A study presented at ISROMAC-4 (Athavale et al. [1992]) of the flows in disc cavities simulated the turbine rotor-stator cavities in a turbomachine. The axial Reynolds number was $\operatorname{Re}_{\chi}=$ $1.17 \times 10^{6}$ and the rotational Reynolds number was $\mathrm{Re}_{\phi}=$ $7.62 \times 10^{6}$; the swirl angle was $30^{\circ}$ at the main-stream inlet flow conditions with $(T, P)=\left(957^{\circ} \mathrm{C}, 0.875 \mathrm{MPa}\right.$; $\left.1754^{\circ} \mathrm{F}, 127 \mathrm{psi}\right)$ and for the coolant flow $(T, P)=(517$ $\left.{ }^{\circ} \mathrm{C}, 0.866 \mathrm{MPa} ; 962^{\circ} \mathrm{F}, 126 \mathrm{psi}\right)$. These results demonstrated a choking vortex at the rim seal interface that, if uncontrolled, blocked the coolant flows with significant heat rise in the cavity. The streamlines for the disc cavity flows at 21, 100, and 189 percent of design (Fig. 37) illustrate how the rim seal "neck" vortex controlled the cavity flows. Windage heat was significant and if unpurged could warp the disc and cause excessive blade rubs into the shroud. At best the condition causes seal wear that significantly increases SFC over time and at worst causes turbine failure through hard rubs or cyclic metal fatigue.

Eccentric rotor simulation: This study did not address the nature of nonuniform or unsteady cooling and heating due to either eccentric rotor operation or engendered vortex formation within the cavity. It is conceivable that periodic hot spots could develop, but they may also develop when the rotor is centered. Small changes (reducing the rotor radius by one-half the shroud tip thickness) caused a raised rotor to reinforce the circulation bubble (close off the purge flow and heat the cavity) and a depressed rotor to weaken the circulation bubble (suck out the purge flow).

The baseline case streamlines illustrate the rim seal "neck" vortex (Fig. 38(a)) and thermal contours (Fig. 38(b)). An increase in rotor radius simulated a forwardfacing step with enhanced vortex strength and significant heating of the rim seal "neck" (Figs. 38(c) and (d)). A decrease in rotor radius simulated a backward-facing step with decreases in vortex strength and decreases in thermal loading in the rim seal "neck" (Figs. 38(e) and (f)).

Interactions of thermal management and engine dynamics. Engine dynamics and thermomechanical mismatching of components play a major role in thermal management: designers are required to provide excessive coolant flows and seal flows to compensate for the thermal unbalance caused by eccentric operations and thermomechanical behavior (e.g., disc warp, low and high cycle fatigue, and unsteady power-stream interactions with the secondary fluid stream). 

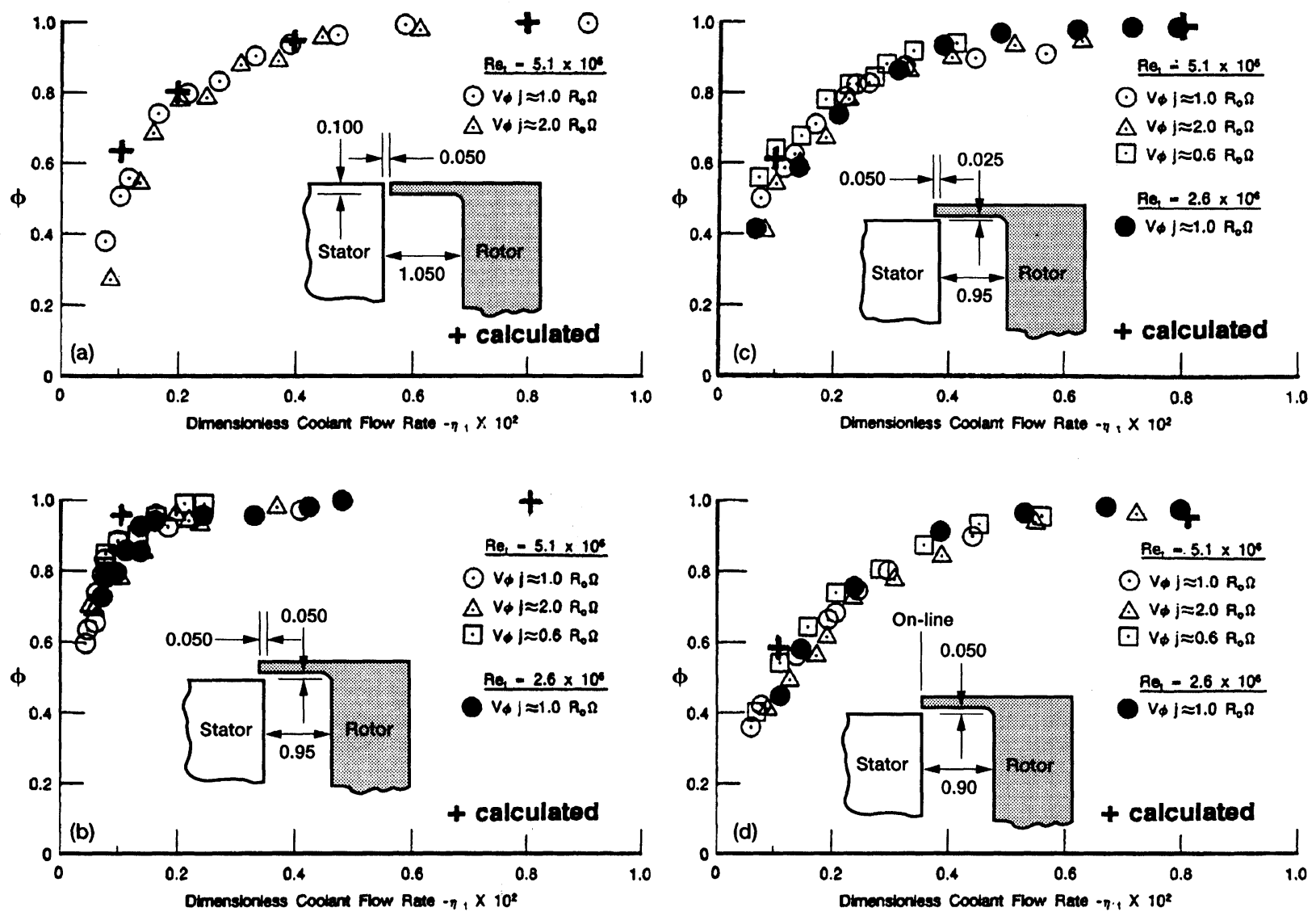

FIGURE 31 Comparison of calculated and experimental data for four UTRC cavity and seal configurations. Calculated results from SCISEAL and experimental data from Graber et al. [1987]. See also figure 27. (a) Configuration 1. (b) Configuration 2. (c) Configuration 3 (baseline). (d) Configuration 4.

TABLE V

Computations Versus Experiments [Cooling effectiveness parameter, $\phi^{\mathrm{a}} ; \mathrm{Re}_{t}=5.0 \times 10^{6}, \mathrm{~V}_{\phi j} \mathrm{R} \Omega$.]

\begin{tabular}{|c|c|c|c|c|}
\hline Configuration & $\begin{array}{c}\text { Dimensionless } \\
\text { coolant flow } \\
\text { parameter, } \\
\eta_{t}\end{array}$ & $\phi$ experimental $^{\mathrm{b}}$ & $\phi$ calculated & $\begin{array}{c}\text { Deviation, } \\
\text { percent }\end{array}$ \\
\hline 1 & $\begin{array}{r}0.001 \\
.002 \\
.004 \\
.008\end{array}$ & $\begin{array}{r}0.53 \\
.77 \\
.94 \\
1\end{array}$ & $\begin{array}{l}0.63 \\
.802 \\
.972 \\
.997\end{array}$ & $\begin{array}{c}19 \\
4 \\
3 \\
-0.3\end{array}$ \\
\hline 2 & $\begin{array}{r}0.001 \\
.008\end{array}$ & 0.89 & $\begin{array}{r}0.95 \\
.99\end{array}$ & $\begin{array}{c}7 \\
-\end{array}$ \\
\hline 3 & $\begin{array}{r}0.001 \\
.008\end{array}$ & $\begin{array}{r}0.57 \\
.98\end{array}$ & $\begin{array}{c}0.611 \\
.99\end{array}$ & $\begin{array}{l}7 \\
1\end{array}$ \\
\hline 4 & $\begin{array}{r}0.001 \\
.008\end{array}$ & $\begin{array}{c}0.5 \\
.98\end{array}$ & $\begin{array}{r}0.589 \\
.965\end{array}$ & $\begin{array}{l}18 \\
-2\end{array}$ \\
\hline
\end{tabular}

${ }^{a}$ Numerical results computed using assumed main-flow conditions with axial velocity ranging from 120 to $150 \mathrm{~m} / \mathrm{s}$. ${ }^{b}$ Experimental data from Graber et al. [1987]. 


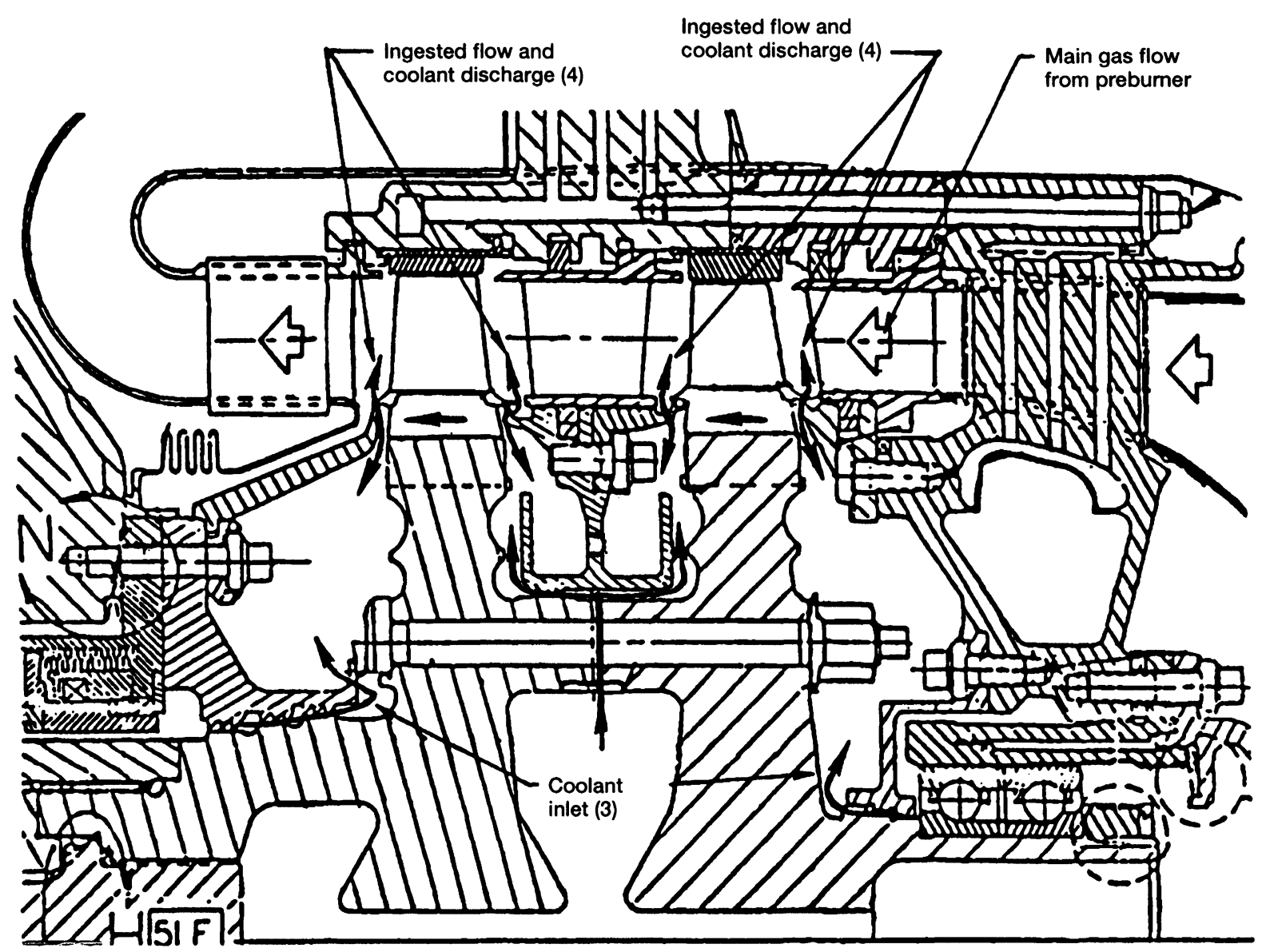

FIGURE 32 Flow path in drive turbine section of current SSME fuel pump. From Daniels and Johnson [1993].

\section{Dynamics}

It is important to realize that the most unstable rotor is one precisely centered and that there exists a singularity in the cylindrical polar coordinate system. It is true that small perturbations are rapidly damped to a stable orbit, but nonetheless they exist and turbomachine jitter is a major problem. Machine dynamics cannot be ignored or placed as secondary: coupled flow mechanisms and proper seal design can affect turbomachine stability because seals exist at many interbearing locations. Indiscriminate tightening of the clearance without regard to the dynamics can render an otherwise stable turbomachine unstable (Millsaps and Martinez-Sanchez [1993]). The industry cannot afford to ignore coupled flow dynamic interactions. Furthermore, acoustic coupling of the cavities can be a major source of both boundary layer control and noise. The term "acoustic" here includes a variety of high-frequency pressure oscillations, some of which affect the rotor (Lucas and Plotkin [1992]).

\section{GOALS: NEAR TERM AND LONG RANGE}

\section{Near-Term Goals}

One near-term goal is to foster direct industrial participation in problem solving. Plans include developing and producing the combined code as a tool for industrial and government use by applying the combined code to experimental data cited herein and other results sufficiently detailed to provide an opportunity for code validation. Then the code will be applied to high-powerdensity turbomachines that potentially will serve as cores for future airbreathing turbomachines. These computations can also be used to define instrumentation positions, proposed SSME-Technology Testbed test (e.g., Fig. 32), where a brush seal is proposed to be used as a coolant flow modulator to provide uniform cooling for the turbine disc of the first-stage fuel turbopump. The test will be coordinated between NASA Lewis, MSFC, 


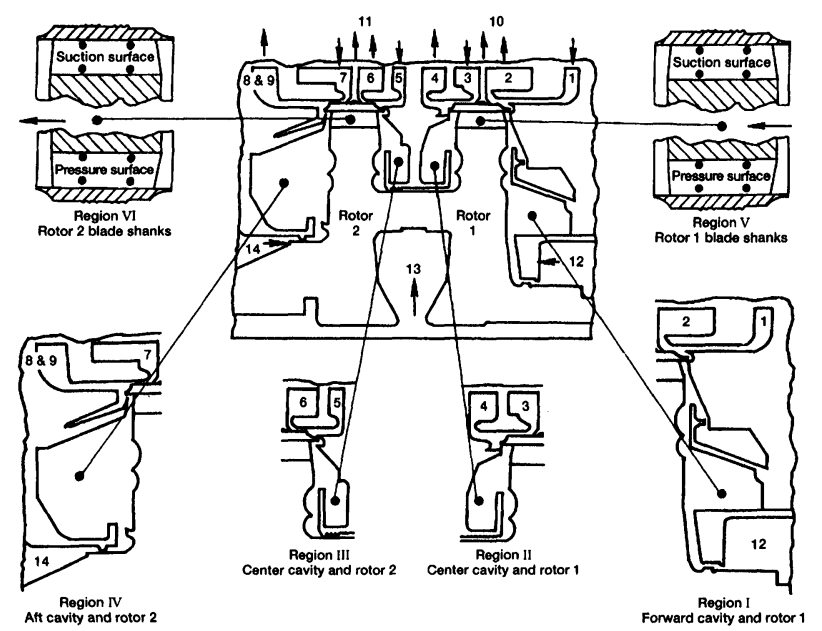

FIGURE 33 Model seal regions and gas source and exit locations. From Daniels and Johnson [1993].

Rocketdyne, and Pratt \& Whitney with the cooperation of others.

\section{Long-Range Goals}

One long-range goal is to examine the feasibility of adapting the high-power-density, SSME-class turbmachines to military airbreathing systems. Another is to coordinate efforts within the rocket turbomachine and airbreathing industries to better distribute secondary flows, to enhance stability, to decrease component size, and to increase speed to over $100000 \mathrm{rpm}$ while reducing the overall cost of operation and manufacturing.

\section{SUMMARY}

The paper gives an overview on the status and scope of fluid bearings, seals, and coupling of the power stream and secondary flow streams for rotating machinery. Several devices are available and can be used advantageously depending on preferred mechanical designs for transporting gases and liquids. Proper attention must be given to rotordynamics to enable high-speed designs and high power densities for advanced aerospace applications.

\section{Fluid Film Bearings}

Hydrodynamic, hydrostatic, foil, and wave bearings enhance stability and have high $D N$ limits relative to conventional journal and contact bearings. For hydro- static bearings to support the load in a low-viscosity working fluid, a high-amplitude hydrodynamic effect is required along with the superimposed small periodic hydrostatic effect (dc with superimposed ac); the hydrostatic effect is the superimposed ripple. For a high viscosity fluid the hydrodynamic effect is small (mostly ac). In hydrostatic pocket bearings shallow pockets develop flow-support "pillows" and recirculation "bubbles." The pressure tends to spike near the Rayleigh step or pinch point in shallow hydrostatic pockets, whereas in deep hydrostatic pockets the pillow and recirculation bubbles advance into the pocket forming two vortics with the injected flow circulating about them. Qualitative agreement with data is encouraging. With foil bearings large radial and axial shaft displacements can be accommodated; $D N$ limits are not yet established. For low-viscosity fluids the load capacity is wanting, but tests with cryogenic turbomachines have been very successful. Damping seal/bearings proved successful in the cryogenic high-pressure pumps of the space shuttle. The damping devices retrofit well in existing designs and open new design opportunities through their dual function as seals and bearings. Robustness, efficiency, compactness, and the containment of catastrophic failure have been addressed. Bulk-flow analyses are available and have spearheaded the designs; computational fluid dynamics is being pursued to improve analytical accuracy and fidelity. Many designs, without the dual function, are already in service; others are being further developed (e.g., as brush seals and foil bearings to meet special requirements). A new bearing, the wave bearing, can support up to 4 times the load with up to 10 times the

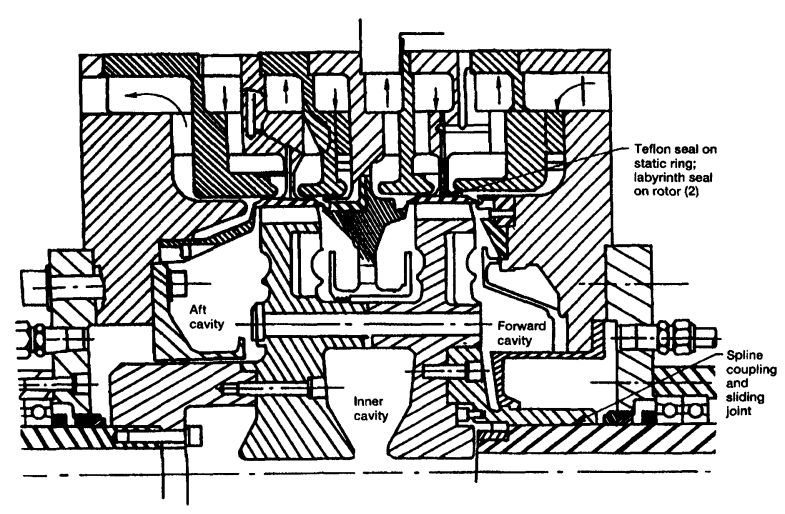

FIGURE 34 Large-scale model of SSME high-pressure fuel turbopump (HPFTP) discs and cavities. From Daniels and Johnson [1993]. 
stiffness of conventional circular journal bearings. Orientation dependencies diminish with number of waves, but the load and stiffness also diminish, with the circular bearing as the limit.

\section{Contact Seals}

Brush seals have consistently outperformed labyrinth seals in low-pressure-drop applications in rig and engine tests and in certified jet engines now flying. For a jet engine, studies indicate potential performance gains of 1 to 2 percent for large engines and 2 to 3 percent for smaller engines by changing labyrinth seals to brush or face seals. However, some design and material improvements are required to retain more of the short-enginehour $(<500 \mathrm{hr})$ performance gaines over labyrinth seals between seal changeouts $(>2000 \mathrm{hr}$ ) over the life of the engine.

\section{Code Development}

The interactive coupling of the power stream or the main stream and the secondary flow streams cannot be neglected. Two separate computational-fluid-dynamics- based computer codes for turbomachinery power streams and secondary flow paths have been developed under NASA projects. The codes, SCISEAL and ADPAC, have been successfully validated for several benchmark problems and demonstrated on various turbomachinery flow problems. The SCISEAL code has been designed to analyze rotating flows in seals, bearings, and secondary passages and to predict seal rotordynamics. It has been successfully used for diverse configurations, such as cylindrical and labyrinth seals, turbine and compressor disc cavities, and rim seals, and for vortex dynamics in blade passages.

ADPAC, which uses a circumferential averaging procedure, has been designed for the treatment of, and successfully applied to, time-dependent flows in multiple rows of stationary and rotating blades. Interactive coupling of the power-stream flow with secondary flow is an enticing area of development. Coupling could be achieved by interfacing two codes, such as ADPAC and SCISEAL, specifically targeted for the two flow streams.

Thermophysical properties are required by the designer and researcher to establish the effects of a real fluid on the component design. Several codes to assist this task are cited herein.

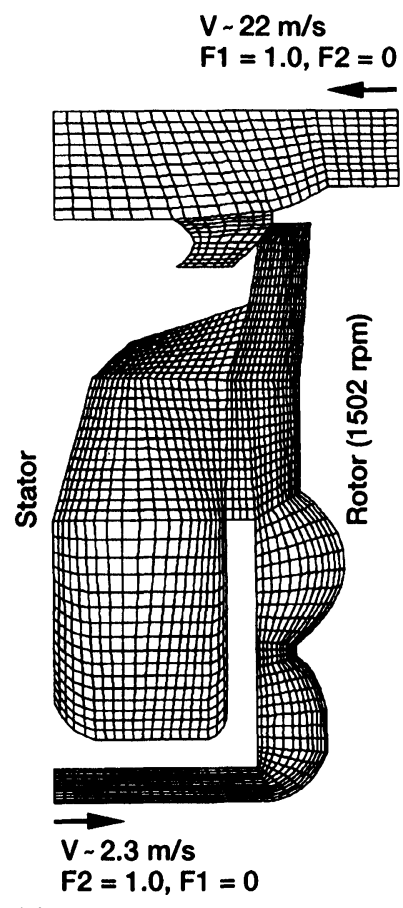

(a)

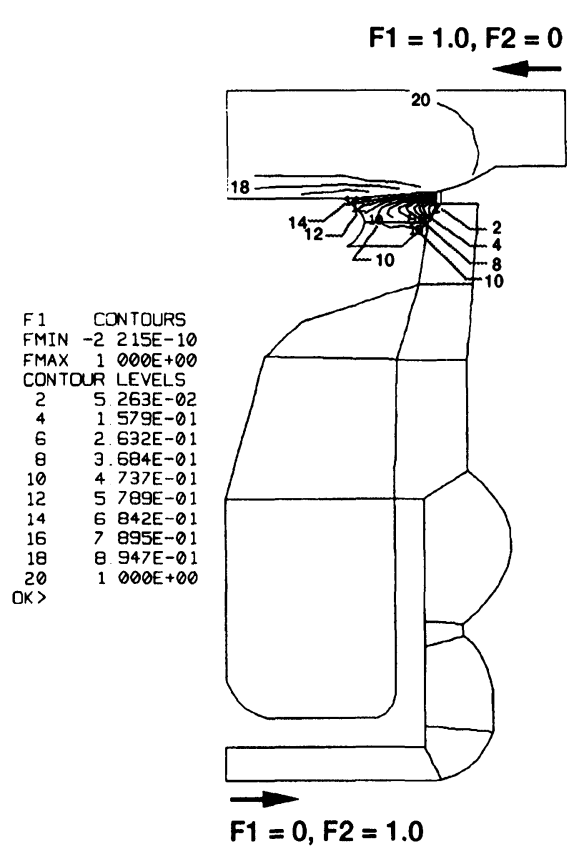

(b)

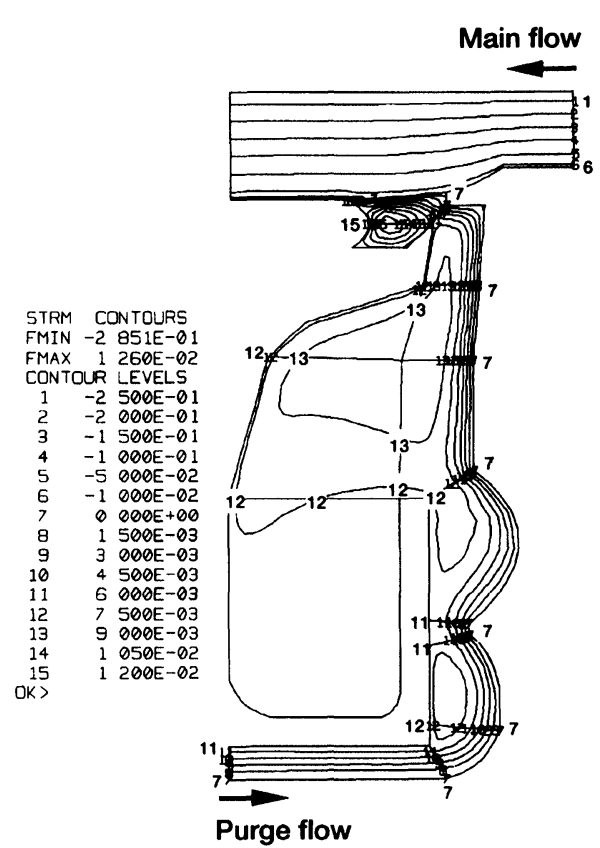

(c)

FIGURE 35 Numerical results for region II center cavity and rotor 1 (see also fig. 33). Main gas flow velocity, $-22 \mathrm{~m} / \mathrm{s}$; purge gas flow velocity, $-2.3 \mathrm{~m} / \mathrm{s}$. (a) Computational grid. (b) Mixture fraction contours. (c) Streamline contours. 


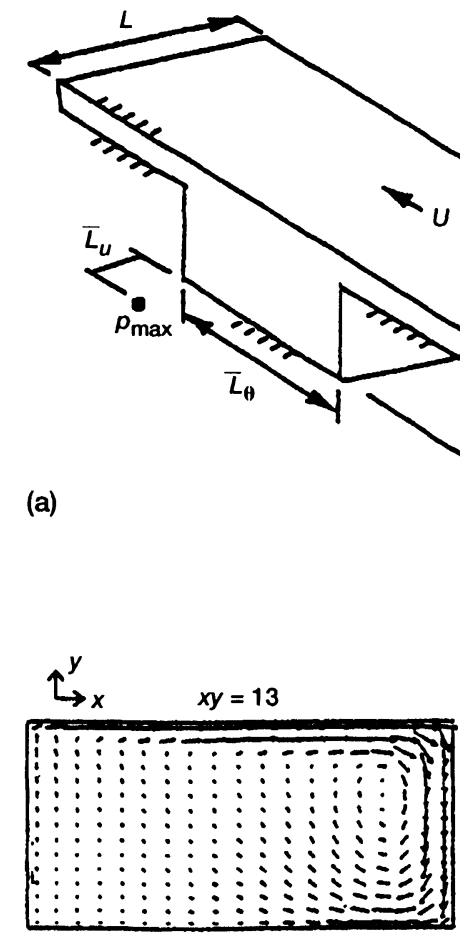

(b)

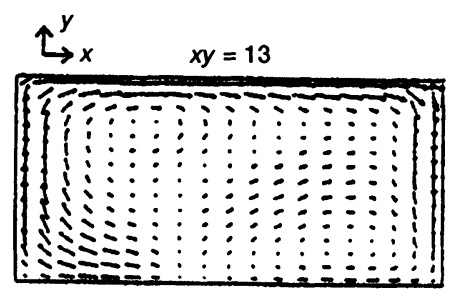

(c)
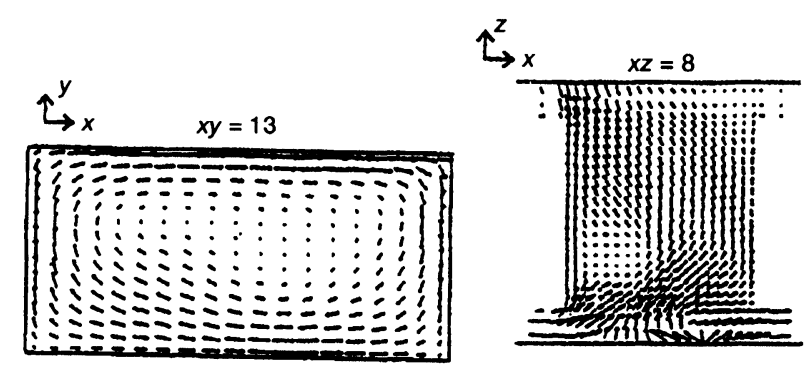

(d)
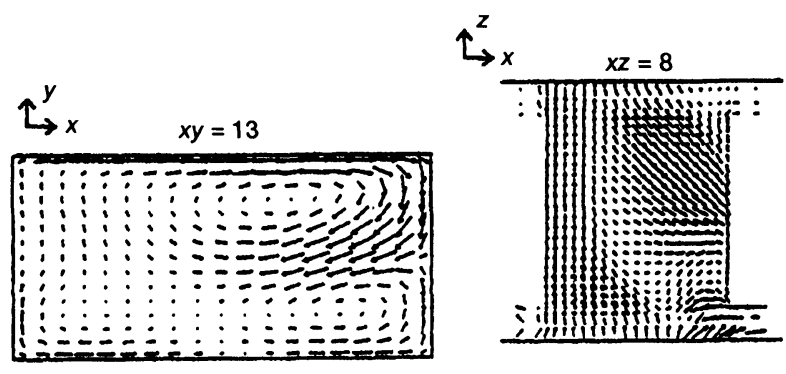

(e)
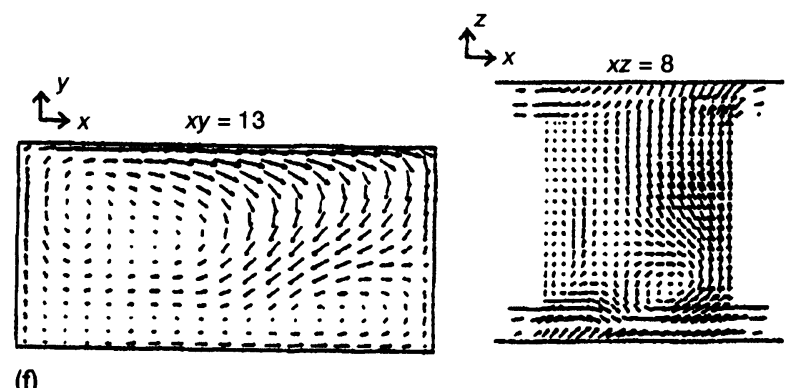

(f)

FIGURE 36 Velocity vector plots for a lid-driven cavity simulating a turbine blade passage at axial plane $x y=13$ (near midplane) and radial plane $x z=8$ (near midplane). (a) Flow geometry. (b) Base test case velocity vector plots at three selected axial planes: $x y=4$ (near inlet), $x y=$ 22 (near exit and radial planes), $x z=1$ (near cavity bottom), $x z=15$ (near cavity top). (c) Inlet corotating vortex injection near pressure side of simulated blade. (d) Inlet corotating vortex injection near suction side of simulated blade. (e) Inlet counterrotating vortex injection near pressure side of simulated blade. (f) Inlet counterrotating vortex injection near suction side of simulated blade.

\section{Closure}

The architecture and fundamentals for interactively coupled power-stream and secondary-flow-stream codes have been initiated. With work these codes will be developed into tools with which to design with confidence. Implementing validated codes to analyze the coupled problems will improve performance, identify design weaknesses prior to expensive testing, and help to reduce design cycle times. Competition and proper marketing will direct future technological preferences based on quality, safety, and low cost. 

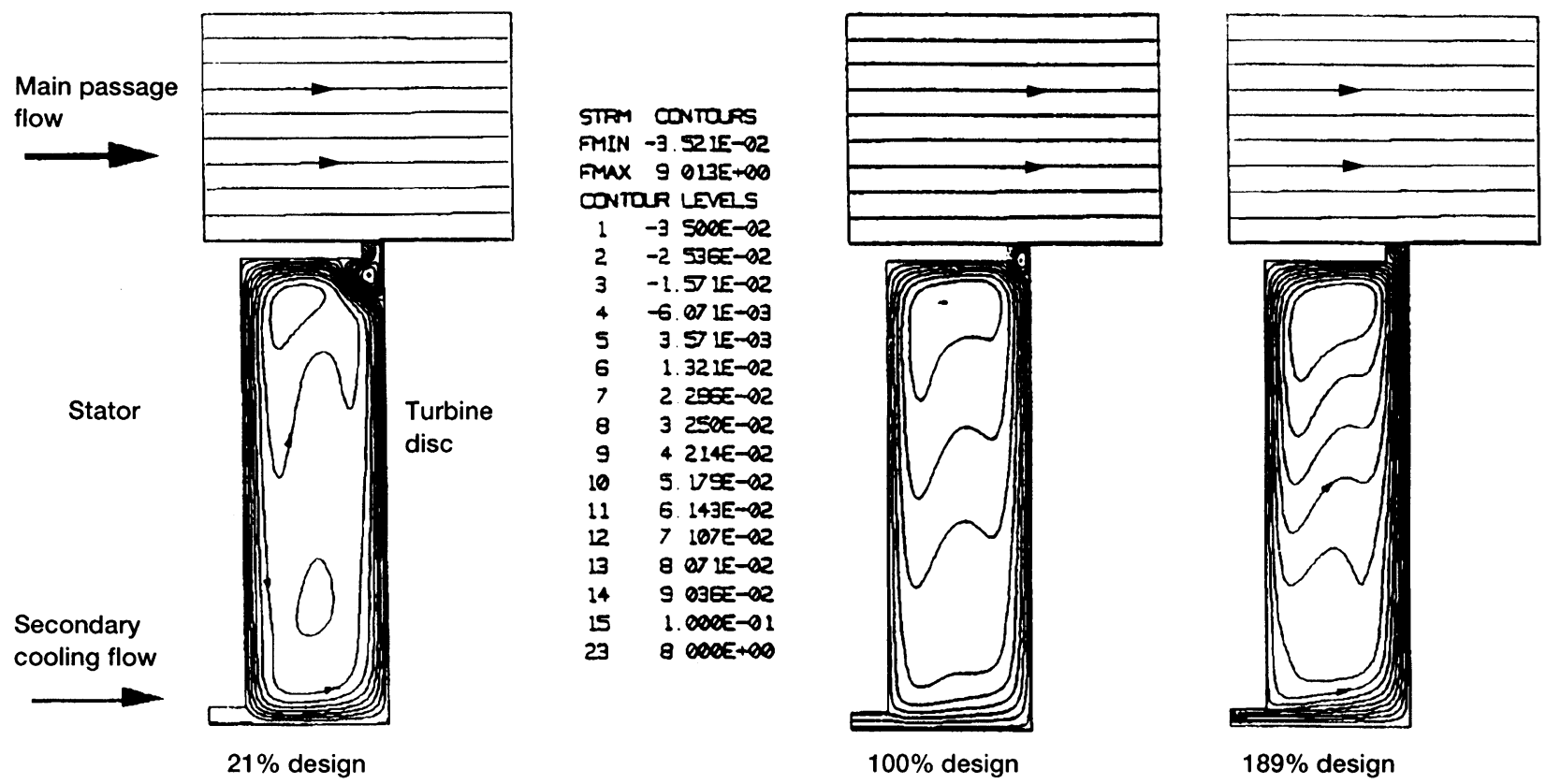

FIGURE 37 Streamlines in turbine disc cavity flow for different secondary cooling rates as directed toward engine thermal management. Note circulation bubble in rim seal "neck" for $21 \%, 100 \%$ and $189 \%$ design flows. Base level case, $C_{q}=7200$.

Nomenclature
$C_{Q}$
$C$
$c$
$D$
$d$
$e$
$F_{1}$
$F_{2}$
$f_{j}$
$h$
$\bar{h}$
$K$
$k_{f}$
$\bar{L}$
$\dot{m}$
$N$
$p$
$p_{m}$
$Q$
$R$
$R e$
$s$
$T$
$t$
$u_{s}$
$V_{\phi}, \mathrm{V}_{\theta}$
$\mathrm{V}$

dimensionless flow parameter, $Q / v R$ mixture concentration

clearance

diameter

dimensionless depth

eccentricity

power-stream (main gas stream) mixture

purge gas mixture

hydrostatic restrictor jet parameter

film thickness

normalized film thickness, $h / c$

fluid film stiffness

foil stiffness

length

mass flow rate

rotor speed, rev/s

pressure

specific pressure

volumetric flow rate

rotor radius

Reynolds number

bump-foil pitch

temperature

thickness

surface speed parameter

circumferential or tangential velocity

velocity

$W$
$x, y, z$
$\alpha$
$\epsilon$
$\eta_{t}$
$\Lambda$
$\mu$
$\nu$
$\rho$
$\phi$
$\theta$
$\omega, \Omega$

load

coordinates

foil-bearing compliance

dimensionless eccentricity, $e / c$

dimensionless coolant flow parameter

bearing parameter

dynamic viscosity

kinematic viscosity,

density

dimensionless cooling effectiveness parameter circumferential coordinate rotor angular speed

\section{Subscripts}

a

$c 1$

$c 1$

$d$

$i$

$\max$

$\min$

$N$

o

$p$

$w$

$w$

$x$
$\theta$ ambient

rotor first critical

thermodynamic critical

downstream

ingested flow

maximum

minimum

nominal

reference condition or inlet

purge flow

upstream

wave

axial

rotational 

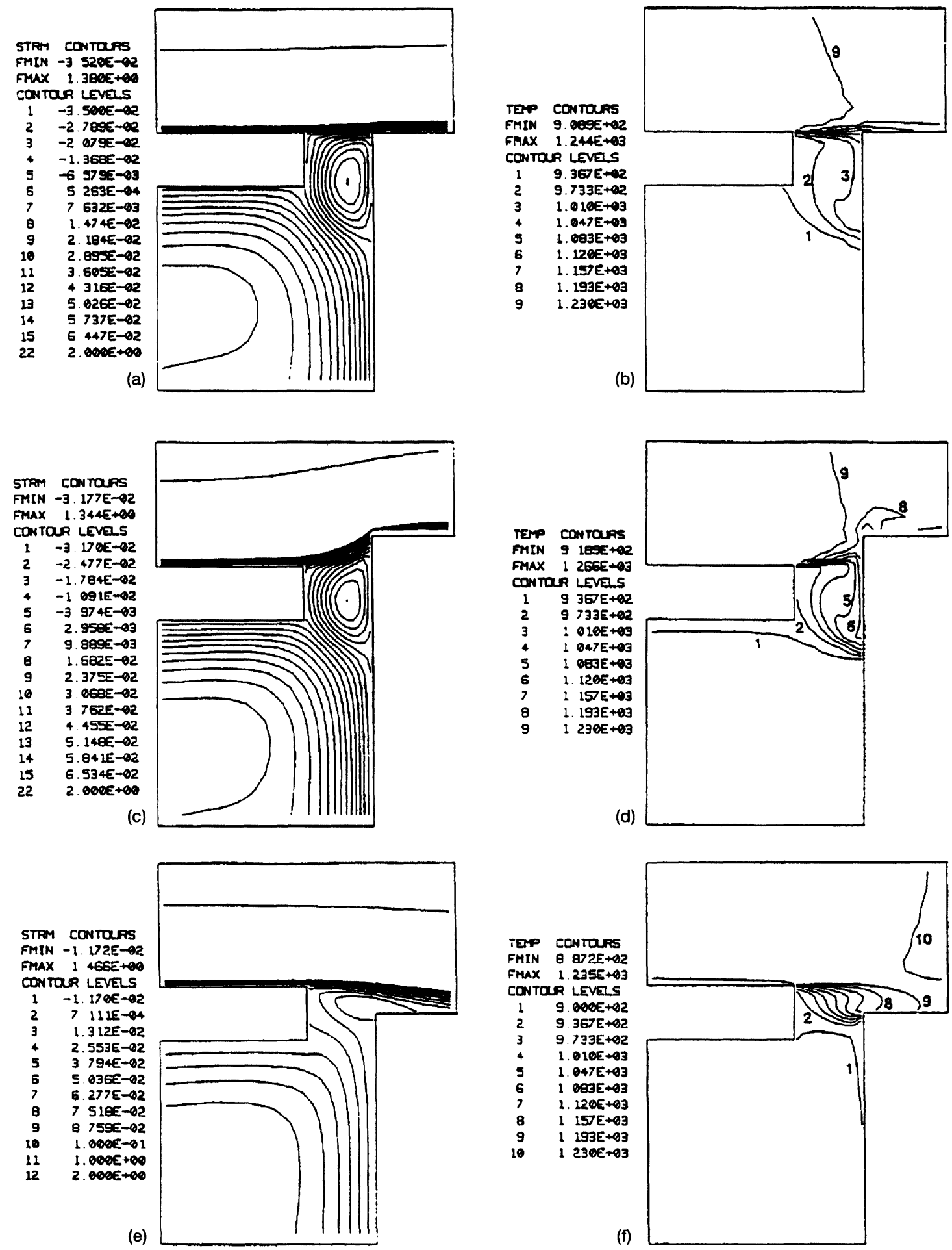

FIGURE 38 Streamline and isotherm contours near rim seal neck for simulated rotor-stator mismatch operation where $\mathbf{R}=\mathbf{R}_{\boldsymbol{\omega}}+\mathrm{e}$. Base level case $C_{q}=7200$ (see also fig. 37). (a) Eccentricity e $=0$, streamlines. (b) $\mathrm{e}=0$ (isotherms). (c) $\mathrm{e}=-0.5$ (seal neck thickness $t$ ), streamlines. (d) $\mathrm{e}=-0.5$ (seal neck thickness $t$ ), isotherms. (e) $\mathrm{e}=0.5$ (seal neck thickness $t$ ), streamlines. (f) $\mathrm{e}=0.5$ (seal neck thickness $t$ ), isotherms. 


\section{References}

Adamczyk, J.J., Beach Celestina, M.L., Beach, T.A., and Barnett, M., 1990. Simulation of Three-Dimensional Viscous Flow Within a Multistage Turbine, Journal of Turbomachinery, Vol. 112.

Athavale, M.M., Przekwas, A.J., and Hendricks, R.C., 1992. A Numerical Study of the Flow Field in Enclosed Turbine Disk Cavities in Gas Turbine Engines, ISROMAC-4, 4th International Symposium on Transport Phenomena and Dynamics of Rotating Machinery, pp. 122-131.

Athavale, M.M., Przekwas, A.J., and Hendricks, R.C., 1993. Driven Cavity Simulation of Turbomachine Blade Flows with Vortex Control. AIAA 93-0390.

Athavale, M.M., Przekwas, A.J., Hendricks, R.C., and Liang, A., 1994. SCISEAL-A Three-Dimensional CFD Code for Accurate Analyses of Fluid Flow and Forces in Seals, 1994 Conference on Advanced Earth-to-Orbit Propulsion Technology, NASA Marshall Space Flight Center, Alabama, May 1994.

Atkinson, E. and Bristol, B.L., 1992. Effects of Material Choices on Brush Seal Performance, Lubrication Engineering, Vol. 48, no. 9, pp. 740-746.

Bache, G., Blumenthal, R., and Swain, R., 1992. A Numerical Procedure for Coupled CFD/Thermal Conduction Analysis of Turbine Disks and Disk Cavities. ISROMAC-4, 4th International Symposium on Transport Phenomena and Dynamics in Rotating Machinery, pp.

Basu, P., Data, A., Derby, R., Johnson, R., Loewenthal, R., and Short, J., 1993. Brush Seal Design Guide, Report WL-TR-93-2029, Texas A\&M University, College Station, TX.

Bayley, F.J. and Owen, J.M., 1970. The Fluid Dynamics of a Shrouded Disk System with a Radial Outflow of Coolant, Journal of Engineering and Power, Vol. 92, pp. 335-342.

Benjamin, T.G. and McConnaughey, P.K., 1991. Navier-Stokes Analysis of Liquid Rocket Engine Disk Cavity, AIAA Paper 91-2361.

Bentley, D.E., Muszynska, A., and Hendricks, R.C., 1986. Bentley Rotordynamics Symposium Proceedings, NASA CP-2409.

Blok, H. and van Rossum, J.J., 1953. The Foil Bearing-A New Departure in Hydrodynamic Lubrication, Lubrication Engineering, Dec., pp. 316-320.

Braun, M.J., Adams, M.L., and Mullen, R.L., 1984-85. Analysis of a Two Row Hydrostatic Journal Bearing with Variable Properties, Inertia Effects, and Surface Roughness, Israel Journal of Technology, Vol. 22, pp. 155-164.

Braun, M.J., Wheeler, R.L., III, and Hendricks, R.C., 1987a. Thermal Shaft Effects on Load-Carrying Capacity of a Fully Coupled, Variable-Properties Cryogenic Journal Bearing, ASLE Transactions, Vol. 30, pp. 282-292.

Braun, M.J., Wheeler, R.L., III, and Hendricks, R.C., 1987b. A Fully Coupled Variable Properties Thermohydraulic Model for a Cryogenic Hydrostatic Journal Bearing, ASME Journal of Tribology, Vol. 109, pp. 405-416.

Braun, M., Canacci, V., and Hendricks, R., 1990. Flow Visualization and Motion Analysis for a Series of Four Sequential Brush Seals, AIAA Paper 90-2482.

Braun, M.J. and Kudriavtsev, V.V., 1993. A Numerical Simulation of a Brush Seal Section and Some Experimental Results, ASME Paper 93-GT-0398.

Braun, M.J., Choy, F.K., Dzodzo, M., and Hsu, J., 1994. Transient and Steady State Dynamic Analysis of a Foil Bearing, ISROMAC-5, 5th International Symposium on Transport Phenomena and Dynamics of Rotating Machinery, May 1994, Maui, HI.

Brennan, J.A., Friend, D.G., Arp, V.D., and McCarty, R.D., 1992. Computer Program for Computing the Properties of Seventeen Fluids, Cryogenics, Vol. 32, no. 2, pp. 212-214.
Carlile, J.A., Hendricks, R.C., and Yoder, D.A., 1992. Brush Seal Leakage Performance with Gaseous Working Fluids at Static and Low Rotor Speed Conditions, presented at 37th International Gas Turbine and Aeroengine Congress and Exposition sponsored by the American Society of Mechanical Engineers, Cologne, Germany, June 1-4. (See also NASA TM-105400.)

Carpino, M., 1990. Overview of Foil Bearing Investigations at Penn State, Advanced Earth-to-Orbit Propulsion Technology, NASA CP3092, pp. 483-490.

Childs, D.W., Vance, J.M., and Hendricks, R.C., eds., 1980. Rotordynamic Instability Problems in High Performance Turbomachinery, NASA CP-2133.

Childs, D.W., Vance, J.M., and Hendricks, R.C., eds., 1982. Rotordynamic Instability Problems in High Performance Turbomachinery, NASA CP-2250.

Childs, D.W., Vance, J.M., and Hendricks, R.C., eds., 1984. Rotordynamic Instability Problems in High Performance Turbomachinery, NASA CP-2338.

Childs, D.W., Vance, J.M., and Hendricks, R.C., eds., 1986. Rotordynamic Instability Problems in High Performance Turbomachinery, NASA CP-2443.

Childs, D.W., Vance, J.M., and Hendricks, R.C., eds., 1988. Rotordynamic Instability Problems in High Performance Furbomachinery, NASA CP-3026.

Childs, D.W., Vance, J.M., and Hendricks, R.C., eds., 1990. Rotordynamic Instability Problems in High Performance Turbomachinery, NASA CP-3122.

Childs, D.W., Vance, J.M., and Hendricks, R.C., eds., 1993. Rotordynamic Instability Problems in High Performance Turbomachinery, NASA CP-3239.

Childs, D. and Hale, K., 1993. A Test Apparatus and Facility To Identify the Rotordynamic Coefficients of High-Speed Hydrostatic Bearings, Rotordynamic Instability Problems in High Performance Turbomachinery, NASA CP-3239, 1994.

Chupp, R.E. and Dowler, C.A., 1991. Performance Characteristics of Brush Seals for Limited Life Engines, ASME Paper 91-GT-281.

Chupp, R.E. and Nelson, P., 1990. Evaluation of Brush Seals for Limited Life Engines, AIAA Paper 90-2140.

Claus, R.W., Evans, A.L., Lytle, J.K., and Nichols, L.D., 1991. Numerical Propulsion System Simulation, Computing Systems in Engineering, Vol. 2, no. 4, pp. 357-364.

Conner, K.J. and Childs, D.W., 1992. Rotordynamic and Leakage Characteristics of a 4-Stage Brush Seal, Report WL-TR-92-2125, Texas A\&M University, College Station, TX.

Daily, J.W. and Nece, R.E., 1960. Chamber Dimension Effects on Induced Flow and Frictional Resistance of Enclosed Rotating Discs, Journal of Basic Engineering, Vol. 82.

Daniels, W.A. and Johnson, B.V., 1993. Experimental Investigation of Turbine Disk Cavity Aerodynamics and Heat Transfer. Contract NAS8-37462, UTRC Report 93-957878-27.

Derby, J. and England, R., 1992. Tribopair Evaluations of Brush Seal Applications, AIAA Paper 92-3715.

Dill, J. and Heshmat, H., 1993. High Speed and Load Capabilities Demonstrated with Aerodynamic Foil Bearings, Mechanical Technology Inc., 93SP2.

Dimofte, F., 1993. A Wave Bearing Concept with Improved SteadyState Performance, NASA CP-3239.

Dimofte, F. and Addy, H.E., 1993. Pressure Measurements of a Three Wave Journal Air Bearing, NASA CP-10136.

DiRusso, E. and Brown, G.V., 1992. Tests of a Cryogenic Magnetic Bearing with Permanent Magnet Bias, Advanced Earth-to-Orbit Propulsion Technology, NASA CP-3174, Vol. II, pp. 491-500. 
Dowson, D., Hudson, J.D., Hunter, B., and March, C.N., 1966-67. An Experimental Investigation of the Thermal Equilibrium of Steadily Loaded Journal Bearings, Proceedings of Institution of Mechanical Engineers, Vol. 181, pt. 3B, pp. 70-80.

Emerson, T., 1978. The Application of Foil Air Bearing Turbomachinery in Aircraft Environmental Control Systems, ASME-78-ENAS-18.

Eshel, A. and Elrod, H.G., Jr., 1965. The Theory of the Infinitely Wide Perfectly Flexible, Self-Acting Foil Bearing, Journal of Basic Engineering, Dec., pp. 831-836.

Ferguson, J.G., 1988. Brushes as High Performance Gas Turbine Seals, ASME Paper 88-GT-182.

Fessler, T.E., 1977. FLUID: A Numerical Interpolation Procedure for Obtaining Thermodynamic and Transport Properties of Fluids, NASA TM X-3572.

Flower, R., 1990. Brush Seal Development System, AIAA Paper 90-2143.

Fowler, J.R., 1987. GASPLUS Users Manual, NASP CR-1012.

Gan, X., Kilic, M., and Owen, J.M., 1993. Flow Between ContraRotating Discs, 38th ASME International Gas Turbine and Aeroengine Congress and Exposition, Cincinnati, OH, 93-GT-286.

Genge, G.G., Saville, M., and Gu, A., 1993. Foil Bearing Performance in Liquid Nitrogen and Liquid Oxygen, AIAA-93-2536.

Ghia, U., Ghia, K.N., and Shin, C.T., 1982. High Resolutions for Incompressible Flow Using the Navier-Stokes Equations and a Multigrid Method. Journal of Computational Physics, Vol. 48.

Gibson, H.G. and Fears, S.D., 1992. Bearing Test Performed in Liquid Oxygen, Advanced Earth-to-Orbit Propulsion Technology, NASA CP-3174, Vol. II, pp. 417-428.

Gilbrech, R.J., Gu, A., Rigney, T., Saville, M., and Rossoni, M., 1993. Liquid Hydrogen Foil-Bearing Turbopump, AIAA-93-2537.

Gordon, S. and McBride, B.J., 1976. Computer Program for Calculation of Complex Chemical Equilibrium Compositions, Rocket Performance, Incident and Reflected Shocks, and Chapman-Jouguet Detonations, NASA SP-273. (See also NASA TM-86885, 1984.)

Graber, D.J., Daniels, W.A., and Johnson, B.V., 1987, Disk Pumping Test, AFWAL-TR-87-2050.

Hall, E.J. and Delaney, R.A., 1993a. Investigation of Advanced Counterrotation Blade Configuration Concepts for High Speed Turboprop Systems, Task 5-Unsteady Counterrotation Ducted Propfan Analysis Computer Program User's Manual, NASA CR-187125.

Hall, E.J., and Delaney, R.A., 1993b. Investigation of Advanced Counterrotation Blade Configuration Concepts for High Speed Turboprop Systems, Task 5-Unsteady Counterrotation Ducted Propfan Analysis Final Report, NASA CR-187126.

Hamrock, B.J., 1991. Fundamentals of Fluid Film Lubrication, NASA RP-1255.

Hannum, N.P. and Nielson, C.E., 1983. The Performance and Application of High Speed Long Life LH2 Hybrid Bearing for Reusable Rocket Engine Turbomachinery, NASA TM-83417.

Hayase, T., Humphrey, J.A.C., and Greif, R., 1990. Numerical Study of Three-Dimensional Flow and Heat Transfer Between a Fixed Outer and Rotating Inner Cylinder with Cavities. 3rd International Symposium on Transport Phenomena and Dynamics of Rotating Machinery, Vol. 1 .

Hendricks, R.C., Peller, I.C., and Baron, A.K., 1973. WASP-A Flexible Fortran IV Computer Code for Calculating Water and Steam Properties, NASA TN D-7391.

Hendricks, R.C., Baron, A.K., and Peller, I.C., 1975. GASP-A Computer Code for Calculating the Thermodynamic and Transport Properties for Ten Fluids: Parahydrogen, Helium, Neon, Methane, Nitrogen, Carbon Monoxide, Oxygen, Fluorine, Argon, and Carbon Dioxide, NASA TN D-7808.

Hendricks, R.C. and Sengers, J.V., 1980. Application of the Principle of Similarity to Fluid Mechanics-Water and Steam: Their Properties and Current Industrial Applications, 9th International Conference on Properties of Steam, J. Straub and K. Scheffler, eds., Pergamon Press, Oxford, (See also NASA TM-79258, 1979.)

Hendricks, R.C., Schlumberger, S., Braun, M.J., Choy, F., and Mullen, R.L., 1991. A Bulk Flow Model of a Brush Seal System, ASME Paper 91-GT-325.

Hendricks, R.C., Carlile, J.A., Liang, A.D., Steinetz, B.M., Easter, B.T., Onstott, J.W., and Howe, H., 1992. Static Brush Seals for Propulsion System Interfaces, Advanced Earth-to-Orbit Propulsion Technology-1992, NASA CP-3174, Vol. 1, pp. 432-439.

Hendricks, R.C., Carlile, J.A., and Liang, A.D., 1993a. Brush Seal Low Surface Speed Hard-Rub Characteristics, AIAA Paper 93-2534.

Hendricks, R.C., Griffin, T.A., Bobula, G.A., Bill, R.C., and Howe H.W., 1993b. Integrity Testing of Brush Seal in Shroud Ring of T-700 Engine, 38th ASME International Gas Turbine and Aeroengine Congress and Exposition, Cincinnati, OH, 93-GT-373.

Hendricks, R.C., 1994. Brush Seal Performance and Durability Issues Based on T-700 Engine Test Results. Accepted for publication in International Journal of Rotating Machinery.

Hendricks, R.C., Griffin, T.A., Csavina, K.R., Pancholi, A., and Sood, D., 1994. Relative Performance Comparison Between Baseline Labyrinth and Dual-Brush Compressor Discharge Seals in a T-700 Engine Test, NASA TM-106360.

Heshmat, H., Walowit, J.A., and Pinkus, O., 1983. Analysis of Gas-Lubricated Foil Journal Bearings, Journal of Lubrication Technology, Vol. 105, no. 10, pp. 647-655.

Heshmat, H., 1993. The Advancement in Performance of Aerodynamic Foil Bearings: High Speed and Load Capacity, STLE/ASME Tribology Conference, Oct. 24-27, 1993, New Orleans, LA

Holle, G.F. and Krishnan, M.R., 1990. Gas Turbine Brush Seal Applications, AIAA Paper 90-2142.

Huzal, D.K. and Huang, D.H., 1992. Modern Engineering for Design of Liquid-Propellant Rocket Engines, Vol. 147, Progress in Astronautics and Aeronautics, A.R. Seebass, ed., AIAA, Washington, DC.

Iwatsubo, T. and Sheng, B.C., 1990. An Experimental Study on the Static and Dynamic Characteristics of Damper Seals, Proceedings of 3rd International Conference on Rotordynamics, Lyon, France, pp. 307-312.

Jacobsen, R.T., Lemmon, E.W., Beyerlein, S.W., Penoncello, S.G., and Ford, M.J., 1991. Personal Computer Programs for Calculating Thermodynamic Properties from Fundamental Equations of State for Fluids of Engineering Interest. Center for Applied Thermodynamic Studies Report 91-1.

Johnson, R.C., 1971. Real Gas Effects in the Flow of Methane and Natural Gas Through Critical Flow Nozzles, NASA TM X-52994.

Khonsari, M.M., 1987. A Review of Thermal Effects in Hydrodynamic Bearings, Part I: Slider/Thrust Bearings; Part II: Journal Bearings, ASLE Transactions, Vol. 30, pp. 26-33.

Liang, A.D. and Hendricks, R.C., 1991. Seals Flow Code Develop ment-1991, NASA CP-10070.

Liang, A.D. and Hendricks, R.C., 1992. Seals Flow Code Development-1992, NASA CP-10124.

Liang, A.D. and Hendricks, R.C., 1993. Seals Flow Code Development-1993, NASA CP-10136.

Licht, L. and Branger, M., 1973a. Design, Fabrication, and Performance of Foil Journal Bearings for the Brayton Rotation Unit, NASA CR-2243.

Licht, L., Branger, M., and Anderson, W.J., 1973b. Gas-Lubricated Foil Bearings for High Speed Turboalternator-Construction and Performance, ASME Paper 73-Lub-5.

Lucas, M.J. and Plotkin, K.J., 1992, Acoustic Characteristics of Turbomachine Cavities, Advanced Earth-to-Orbit Propulsion Technologies, NASA CP-3174, Vol. II, pp. 562-568.

McConnaughey, H.V., Leopard, J.L., and Lightfoot, R.M., 1992. Test 
Results of Highly Instrumented Space Shuttle Main Engine, AIAA 92-3452.

Millsaps, K.T. and Martinez-Sanchez, M., 1993. Rotordynamic Forces in Labyrinth Seals: Theory and Experiment, NASA CP-3239.

Mollerup, J., 1975. Correlated and Predicted Thermodynamic Properties of LNG and Related Mixtures in the Normal and Critical Regions, Advances in Cryogenic Engineering, K.D. Timmerhaus, ed., Vol. 20, pp. 172, 194.

Morrison, G.L., Shresta, S., DeOtte, R.E., Jr., and Johnson, M.C., 1994 Upstream Swirl Effects Upon the Flow Field Inside a Centered Annular Seal. ISROMAC-5, 5th International Symposium on Transport Phenomena and Dynamics of Rotating Machinery, May, Maui, HI. Nemeth, Z., 1994. Private communication.

Nichols, L.D. and Chamis, C.C., 1991. Numerical Propulsion System Simulation: An Interdisciplinary Approach, AIAA-91-2554.

Nolan, S.A. and Genge, G., 1993. Hot Fire Testing of an SSME HPOTP with Annular Hydrostatic Bearings, AIAA 93-2356.

Oates, G.C., 1984. Aerothermodynamics of Gas Turbine and Rocket Propulsion, AIAA Education Series, J.S. Przemieniecki, ed., AIAA, New York.

O’Connor, L, 1993. Fluid-Film Foil Bearings Control Engine Heat. Mechanical Engineering, Vol. 115, no. 5, pp. 72-75.

Petchenev, A., Goldman, P., Muszynska, A., and Bentley, D.E., 1992. The Analytical Description of the Fluid Film Bearings of Finite Length, Bentley Rotordynamics Research Corporation, BRDRCReport \# 5.

Phadke, U.P. and Owen, J.M., 1983. An Investigation of Ingress for an 'Air-Cooled' Shrouded Rotating Disk System with Radial-Clearance Seals, Journal of Engineering and Power, Vol. 105, pp. 178-183.

Ping, J.P. and Carpino, M., 1993. Calculation of Stiffness and Damping Coefficients for Elastically Supported Gas Foil Bearings, Journal of Tribology, Vol. 115, no. 1, pp. 20-27.

Pinkus, O., 1990, Thermal Aspects of Fluid Film Tribology, ASME Press, New York.

Proctor, M.P., Walker, J.F., Perkins, H.D., Hoopes, J.F., and William- son, S.G., 1994. Brush Seals for Cryogenic Applications Performance, Stage Effects, and Preliminary Wear Results in $\mathrm{LN}_{2}$ and $\mathrm{LH}_{2}$. To be published as NASA TP.

San Andres, L., Yang, L., and Childs, D.W., 1992. Thermohydrodynamic Analysis of Cryogenic Liquid Annular Seals, Advanced Earthto-Orbit Propulsion Technology, NASA CP-3174, Vol. 1, p. 468.

San Andres, L., 1993. Dynamic Force Response of Spherical Hydrostastic Journal Bearing for Cryogenic Applications, Rotordynamic Instability Problems in High Performance Turbomachinery, NASA CP-3239.

Saville, M., Gu, A., and Capaldi, R., 1991. Liquid Hydrogen Turbopump Foil Bearing, AIAA Paper 91-2108.

Schmied, J., 1991. Rotordynamic Aspects of a New Hermetically Sealed Pipeline Compressor, Proc. 20th Turbomachinery Symposium, pp. 11-17.

Sutton, G.P., 1986. Rocket Propulsion Elements, John Wiley \& Sons, New York.

Tam, L.T., Przekwas, A.J., Muszynska, A., Hendricks, R.C., Braun, M.J., and Mullen, R.L., 1988. Numerical and Analytical Study of Fluid Dynamic Forces in Seals and Bearings, Journal of Vibration, Acoustics, Stress, and Reliability in Design, ASME, Vol. 110, pp. 315-325.

von Pragenau, G.L., 1982. Damping Seals for Turbomachinery, NASA TP-1987.

von Pragenau, G.L., 1985. Turbomachinery Whirl Elimination with Damping Seals, Research and Technology FY 1985 Annual Report, Marshall Space Flight Center, pp. 79-81.

von Pragenau, G., 1992. From Labyrinth Seals to Damping Seals/ Bearings, ISROMAC-4, 4th International Symposium on Transport Phenomena and Dynamics of Rotating Machinery, pp. 277, 285.

Zaretsky, E.V., ed., 1992. STLE Life Factors for Rolling Bearings, STLE SP-34, Society of Tribologists and Lubrication Engineers, Park Ridge, IL.

Zaretsky, E.V., ed., 1994. Tribology for Aerospace Applications. To be published. 

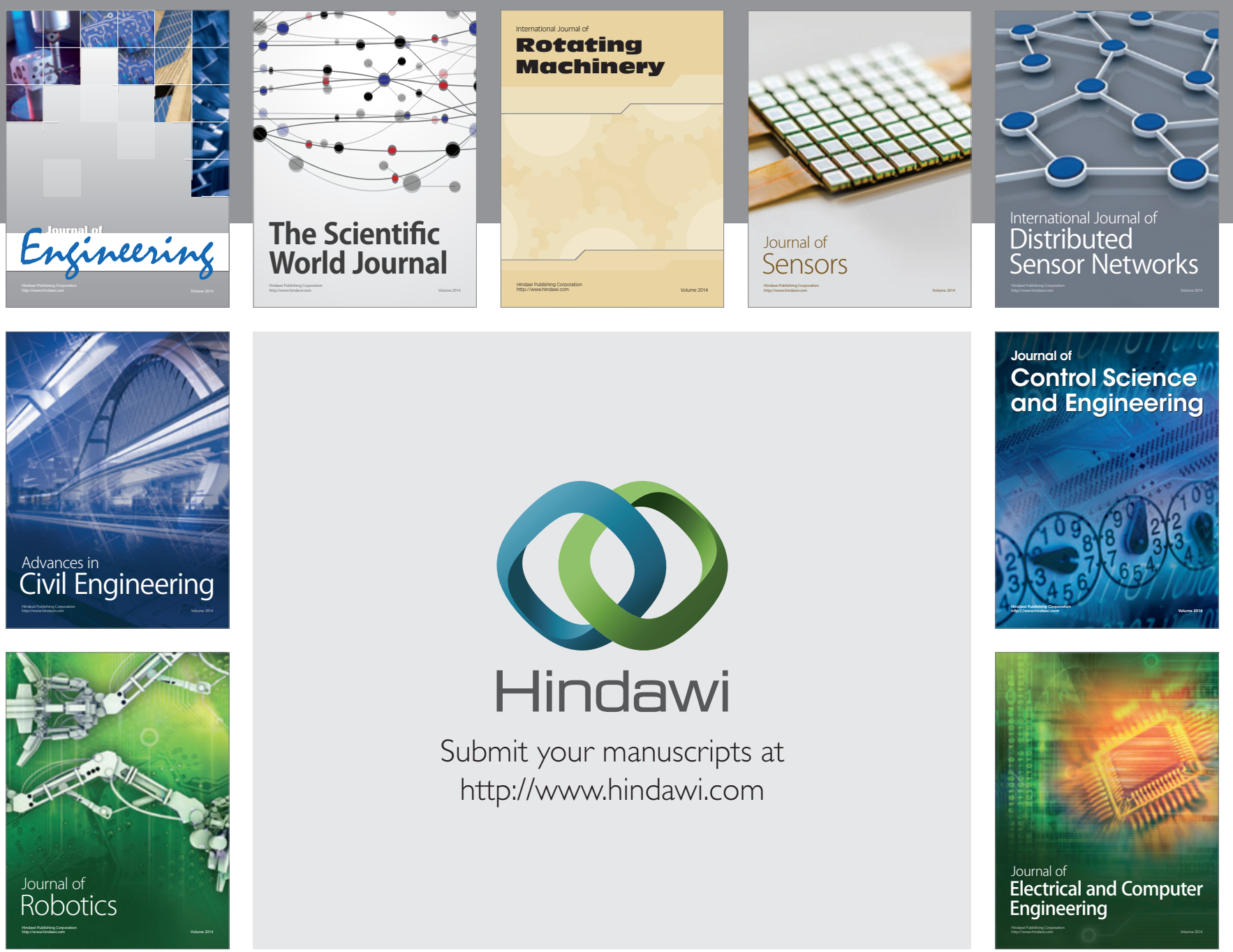

Submit your manuscripts at

http://www.hindawi.com
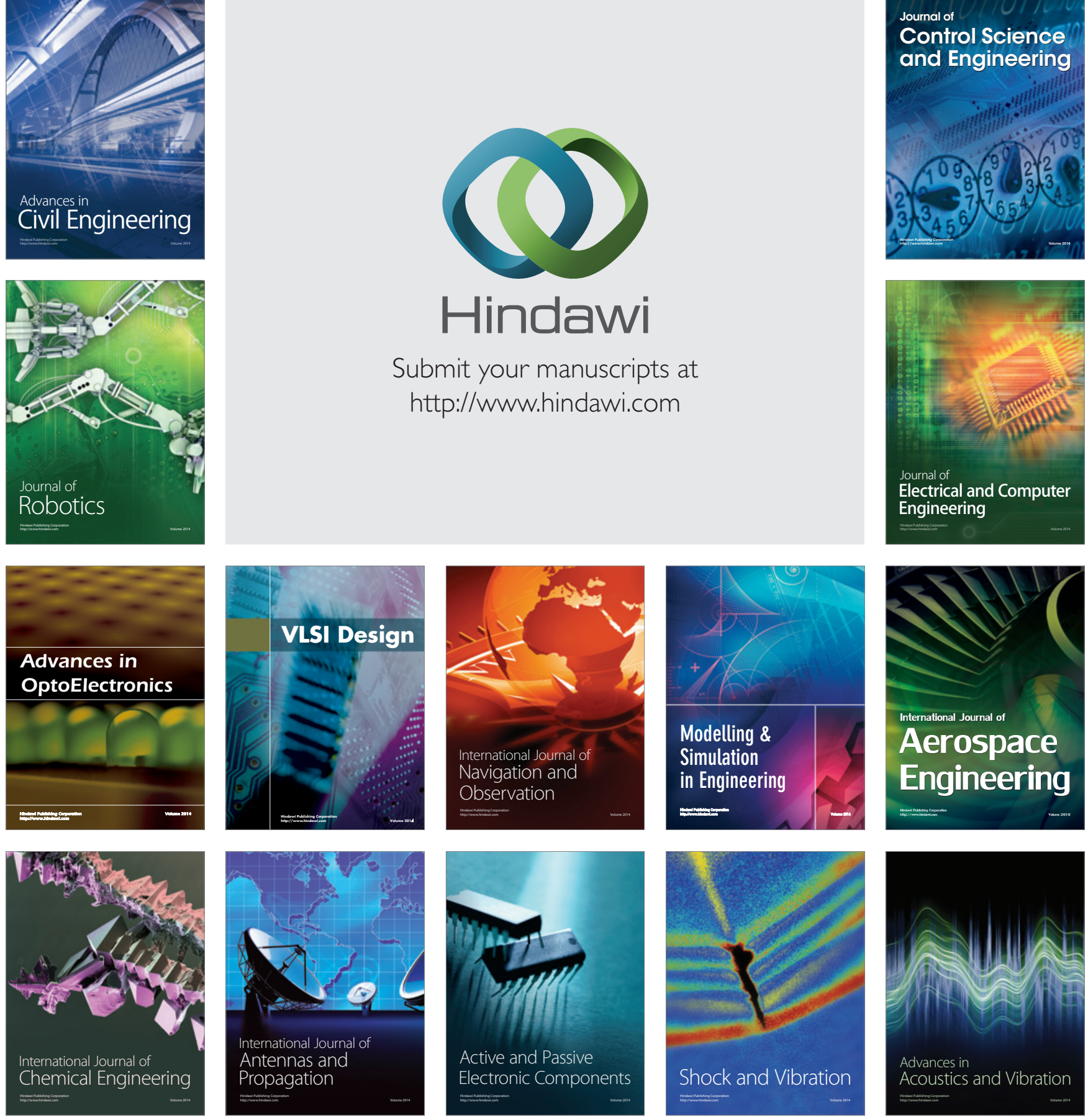